\title{
Intermolecular Oxidative Friedel-Crafts Reaction Triggered Ring Expansion Affording 9,10-Diarylphenanthrenes
}

Lu Yang, ${ }^{\dagger}$ Ilya. D. Gridnev,${ }^{\dagger}$ Masahiro Terada, ${ }^{\dagger}$ and Tienan $\mathrm{Jin}^{\star, \neq}$

$\dagger \quad$ Department of Chemistry, Graduate School of Science

Tohoku University

6-3 Aramaki Azaaoba Aoba-ku, Sendai 980-8578

$\ddagger \quad$ Research and Analytical Center for Giant Molecules, Graduate School of Science

Tohoku University

6-3 Aramaki Azaaoba Aoba-ku, Sendai 980-8578

E-mail: tetsuo.kin.a6@tohoku.ac.jp 


\section{Table of Contents}

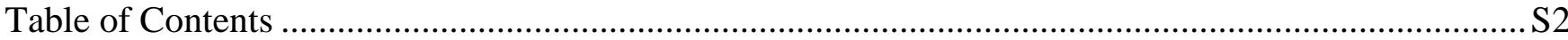

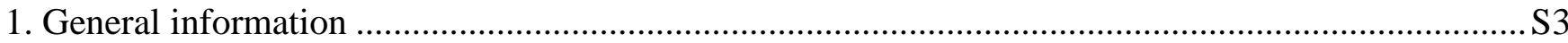

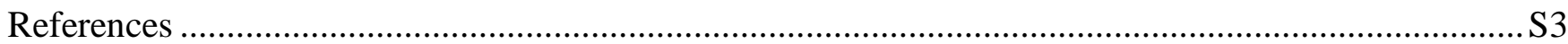

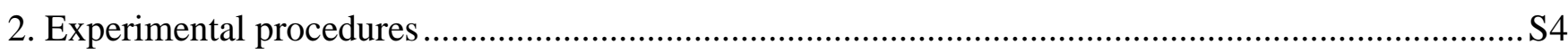

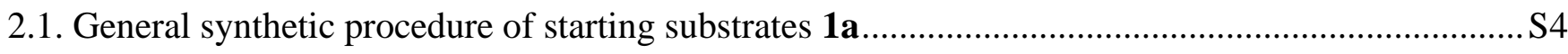

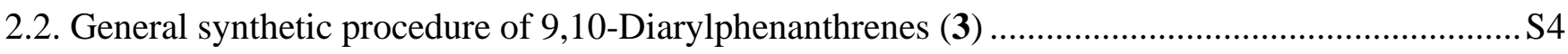

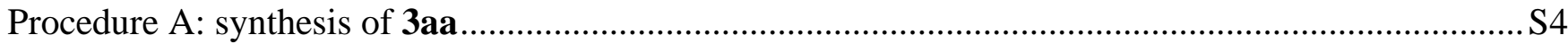

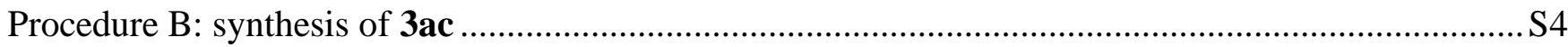

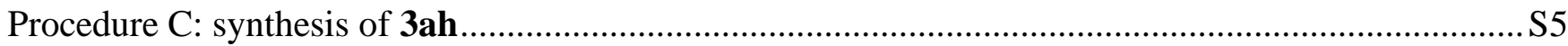

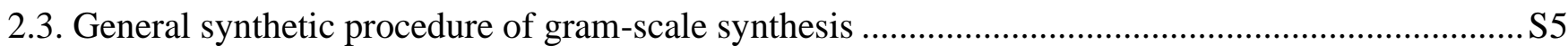

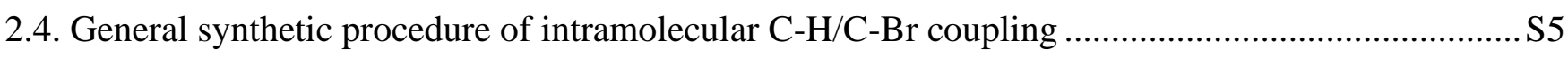

2.5. General synthetic procedure of one-pot oxidation process ...................................................... 6

2.6. General synthetic procedure of two-fold tandem oxidation to access 5ah ...................................S6

2.7. General synthetic procedure of two-fold tandem oxidation to access $\mathbf{5 l j}$.....................................S6

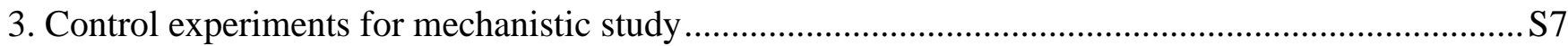

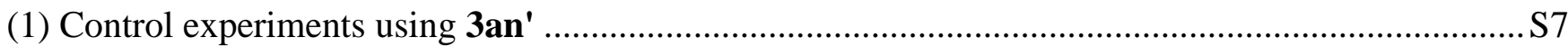

(2) Reaction of 1a with $\mathbf{2 n}$ under the standard conditions in the presence of radical scavenger .............S7

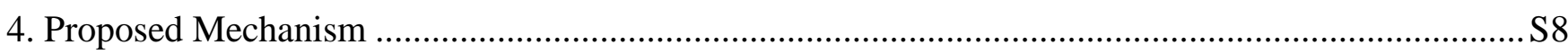

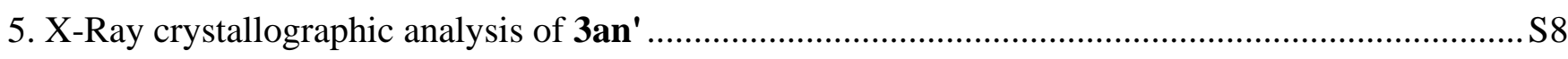

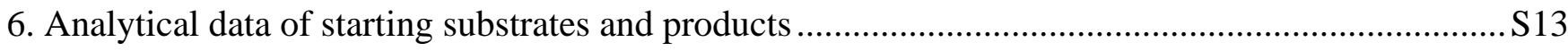

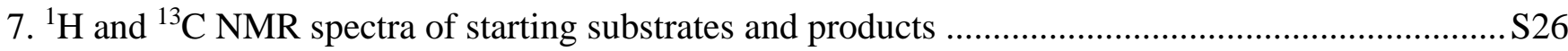

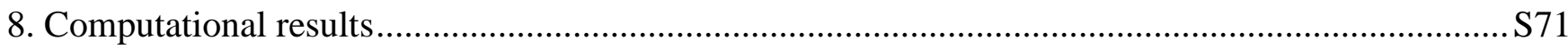




\section{General information}

${ }^{1} \mathrm{H}$ NMR and ${ }^{13} \mathrm{C}$ NMR spectra were recorded on JEOL JNM AL $400(400 \mathrm{MHz})$ and JEOL JNM AL 600 (600 MHz) spectrometers. ${ }^{1} \mathrm{H}$ NMR spectra are reported as follows: chemical shift in ppm $(\delta)$ relative to the chemical shift of $\mathrm{CDCl}_{3}$ at $7.26 \mathrm{ppm}$, multiplicities $(\mathrm{s}=$ singlet, $\mathrm{d}=$ doublet, $\mathrm{t}=$ triplet, $\mathrm{q}=$ quartet, $\mathrm{m}=$ multiplet, and $\mathrm{br}=$ broadened), and coupling constants $(\mathrm{Hz}) .{ }^{13} \mathrm{C} \mathrm{NMR}$ spectra were recorded on JEOL JNM AL $400(100 \mathrm{MHz})$ and JEOL JNM AL $600(150.0 \mathrm{MHz})$ spectrometers with complete proton decoupling, and chemical shift reported in ppm (ס) relative to the central line for $\mathrm{CDCl}_{3}$ at $77 \mathrm{ppm}$. High resolution mass spectra were obtained on a BRUKER APEXIII spectrometer and JEOL JMS-700 MStation operator. Flash column chromatography was performed on silica gel $60 \mathrm{~N}$ (spherical, neutral, 40-50 $\mu \mathrm{m}$; Kanto Chemical Co., Inc.). Analytical thin-layer chromatography (TLC) was performed on $0.2 \mathrm{~mm}$ precoated plate Kieselgel 60 F254 (Merck).

Materials: Materials were purchased from Wako Pure Chemical Industries, Ltd., Tokyo Chemical Industry Co., LTD., Aldrich Inc., and Kanto Chemical Co., Inc. and used as received. The starting substrates $\mathbf{2 m}$ was prepared following the reported literatures. ${ }^{[1]}$ The structure of 3an' was confirmed by means of X-ray crystallography. CCDC 2027514 contains the supplementary crystallography data of 3an'. These data can be obtained free of charge from The Cambridge Crystallographic Data Center. The structures of starting substrates and products $1-5$ were determined by ${ }^{1} \mathrm{H},{ }^{13} \mathrm{C}$ NMR, high resolution mass spectrometry (HRMS), and reported literatures. ${ }^{[1}$ 14]

Computational details: Computations were carried out using the range separated hybrid functional with damped atom-atom dispersion ( $\omega B$ 97XD) $)^{15}$ as implemented in the GAUSSIAN 16 software package. ${ }^{[16]}$ All atoms were modeled at the $6-31 \mathrm{G}(\mathrm{d}, \mathrm{p})$ basis set. ${ }^{[17]}$ Non-specific solvation was introduced by using the SMD continuum model for all optimizations and frequency computations ${ }^{[16]}$ $\left(2,2,2\right.$-trifluoroethanol). ${ }^{[18]}$

\section{References}

[1] N. N. Pham, S. Parpart, S. Grigoryan, T. N. Ngo, T. T. Dang, T. V. Ghochikyan, A. S. Saghyan, P. Ehlers, P. Langer, Eur. J. Org. Chem. 2017, 538-550.

[2] X. Zhang, Z. Xu, W. Si, K. Oniwa, M. Bao, Y. Yamamoto, T. Jin, Nat. Commun. 2017, 8, 15073.

[3] T. S. Navale, K. Thakur, R. Rathore, Org. Lett. 2011, 13, 1634-1637.

[4] J. Yu, H. Yan, C. Zhu, Angew. Chem. Int. Ed. 2016, 55, 1143-1146.

[5] M. Shimizu, I. Nagao, Y. Tomioka, T. Hiyama, Angew. Chem. Int. Ed. 2008, 47, 8096-8099.

[6] R. C. Larock, Q. Tian, J. Org. Chem. 2001, 66, 7372-7379.

[7] M. J. Tanga, J. E. Bupp, J. Org. Chem. 1993, 58, 4173-4174.

[8] C. Do, J. Hatfield, S. Patel, D. Vasudevan, C. Tirla, N. S. Mills, J. Org. Chem. 2011, 76, 181-187.

[9] N. Chernyak, V. Gevorgyan, J. Am. Chem. Soc. 2008, 130, 5636-5637.

[10] M. Paraja, C. Valdés, Org. Lett. 2017, 19, 2034-2037.

[11] Y. Xia, P. Qu, Z. Liu, R. Ge, Q. Xiao, Y. Zhang, J. Wang, Angew. Chem. Int. Ed. 2013, 52, 2543-2546.

[12] M. Shimizu, I. Nagao, Y. Tomioka, T. Hiyama, Angew. Chem. Int. Ed. 2008, 47, 8096-8099.

[13] M. Iwasaki, Y. Araki, Y. Nishihara, J. Org. Chem. 2017, 82, 6242-6258.

[14] J. Yan, N. Yoshikai, Org. Lett. 2017, 19, 6630-6633.

[15] J.-D. Chai, M. Head-Gordon, Phys. Chem. Chem. Phys. 2008, 10, 6615

[16] Gaussian 16, Revision A.03, M. J. Frisch, G. W. Trucks, H. B. Schlegel, G. E. Scuseria, M. A. Robb, J. R. Cheeseman, G. Scalmani, V. Barone, G. A. Petersson, H. Nakatsuji, X. Li, M. Caricato, A. V. Marenich, J. Bloino, B. G. Janesko, R. Gomperts, B. Mennucci, H. P. Hratchian, J. V. Ortiz, A. F. Izmaylov, J. L. Sonnenberg, D. Williams-Young, F. Ding, F. Lipparini, F. Egidi, J. Goings, B. Peng, A. Petrone, T. Henderson, D. Ranasinghe, V. G. Zakrzewski, J. Gao, N. Rega, G. Zheng, W. Liang, M. Hada, M. Ehara, K. Toyota, R. Fukuda, J. Hasegawa, M. Ishida, T. Nakajima, Y. Honda, O. Kitao, H. Nakai, T. Vreven, K. Throssell, J. A. Montgomery, Jr., J. E. Peralta, F. Ogliaro, M. J. Bearpark, J. J. Heyd, E. N. Brothers, K. N. Kudin, V. N. Staroverov, T. A. Keith, R. Kobayashi, J. Normand, K. Raghavachari, A. P. Rendell, J. C. Burant, S. S. Iyengar, J. Tomasi, M. Cossi, J. M. Millam, M. Klene, C. Adamo, R. Cammi, J. W. Ochterski, R. L. Martin, K. Morokuma, O. Farkas, J. B. Foresman, and D. J. Fox, Gaussian, Inc., Wallingford CT, 2016.

[17] a) R. Ditchfield, W. J. Hehre, J. A. Pople, J. Chem. Phys. 1971, 54, 724; b) W. J. Hehre, R. Ditchfield, J. A. Pople, J. Chem. Phys. 1972 56, 2257; c) P. C. Hariharan, J. A. Pople, Theor. Chim. Acta 1973, 28, 213; d) P. C. Hariharan, J. A. Pople, Mol. Phys. 1974, 27, 209; e) M. S. Gordon, Chem. Phys. Lett. 1980, 76, 163.

[18] A. V. Marenich, C. J. Cramer, D. G. Truhlar, J. Phys. Chem. B 2009, 113, 6378. 


\section{Experimental procedures}

\subsection{General synthetic procedure of starting substrates $1 \mathrm{a}$}

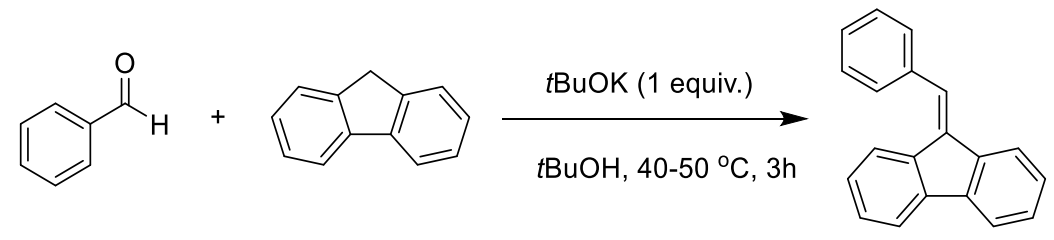

$1 \mathrm{a}, 90 \%$

To a suspension of $9 \mathrm{H}$-fluorene $(498.7 \mathrm{mg}, 3 \mathrm{mmol}$, purchased from TCl) in $t-\mathrm{BuOH}(15 \mathrm{~mL})$ in a $50 \mathrm{~mL}$ flask was added $t$-BuOK (336.6 $\mathrm{mg}, 3 \mathrm{mmol}$ ), which was stirred at $40^{\circ} \mathrm{C}$ in oil bath for $30 \mathrm{~min}$. Then benzaldehyde ( $318.4 \mathrm{mg}, 3 \mathrm{mmol}$, purchased from Aldrich) was added and the reaction mixture was allowed to warm to $50{ }^{\circ} \mathrm{C}$ and stirred for $3 \mathrm{~h}$. After monitoring with TLC, the reaction mixture was extracted with dichloromethane and the organic layer was dried with $\mathrm{Na}_{2} \mathrm{SO}_{4}$. After filtration and evaporation, the residue was purified through silica gel chromatography using hexane/DCM (10/1) as eluents to give the corresponding product 1 a in $90 \%$ isolated yield as a white solid $(686.7 \mathrm{mg})$. The structure of 1a was determined by ${ }^{1} \mathrm{H}$ and ${ }^{13} \mathrm{C}$ NMR spectra, and HRMA compared with the literature ${ }^{[2]}$.

\subsection{General synthetic procedure of 9,10-Diarylphenanthrenes (3)}

\section{Procedure A: synthesis of $3 a a$}

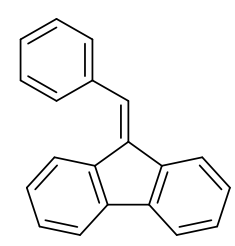

$1 \mathbf{a}$<smiles>Cc1ccccc1C</smiles>

$2 \mathbf{a}$

(3.0 equiv)

\section{$\frac{\text { DDQ (1.5 equiv) }}{\text { TFA, rt, } 4 \mathrm{~h}}$}

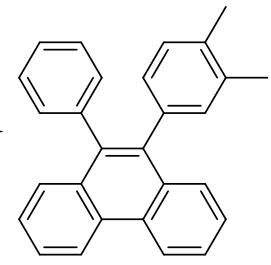

3aa, $95 \%$

To a trifluoroacetic acid (TFA) (0.6 mL) solution of 9-Benzylidene-9H-fluorene (1a) $(25.4 \mathrm{mg}, 0.1 \mathrm{mmol})$ and o-xylene (2a) (31.9 mg, $0.3 \mathrm{mmol}$ ) was added 2,3-dichloro-5,6-dicyano-p-benzoquinone (DDQ) (33.8 mg, $0.15 \mathrm{mmol}$ ). The mixture in a $3 \mathrm{~mL}$ reactor vial was stirred at room temperature for $4 \mathrm{~h}$. After cooling to room temperature, the resulting reaction mixture was quenched by pouring into saturated aqueous $\mathrm{NaHCO}_{3}(2 \mathrm{~mL})$. The organic layer was separated and the aqueous layer was extracted with dichloromethane $(2 \mathrm{x}$ $1 \mathrm{~mL}$ ). Combined organic layers were washed with water and brine, and dried over anhydrous $\mathrm{MgSO}_{4}$. After concentration, the resulting residue was purified by a short pad of silica gel using a 1:10 mixture of DCM and hexane, affording the corresponding product 9-(3,4dimethylphenyl)-10-phenylphenanthrene (3aa) in 95\% yield (34.1 mg) as white solid.

Procedure B: synthesis of 3ac

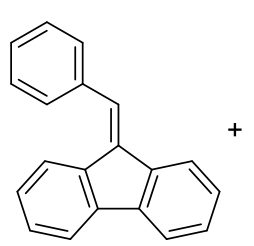

$1 \mathrm{a}$<smiles>c1ccc(-c2ccccc2)cc1</smiles>

2c

(3.0 equiv)

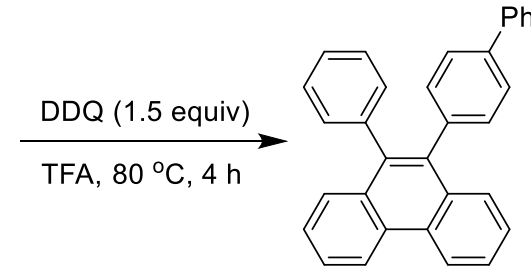

3ac, $71 \%$

To a trifluoroacetic acid (TFA) (0.6 mL) solution of 9-benzylidene-9H-fluorene (1a) (25.4 mg, $0.1 \mathrm{mmol})$ and biphenyl (2c) (46.3 mg, 0.3 $\mathrm{mmol}$ ) was added 2,3-dichloro-5,6-dicyano-p-benzoquinone (DDQ) (33.8 $\mathrm{mg}, 0.15 \mathrm{mmol}$ ). The mixture in a $3 \mathrm{~mL}$ reaction vial was heated on an aluminum-block at $80{ }^{\circ} \mathrm{C}$ for $4 \mathrm{~h}$. After cooling to room temperature, the resulting reaction mixture was quenched by 
pouring into saturated aqueous $\mathrm{NaHCO}_{3}(2 \mathrm{~mL})$. The organic layer was separated and the aqueous layer was extracted with dichloromethane $(2 \times 1 \mathrm{~mL})$. Combined organic layers were washed with water and brine, dried over anhydrous $\mathrm{MgSO}_{4}$. After concentration, the resulting residue was purified by a short pad of silica gel using 1:10 mixture of DCM and hexane, affording the corresponding product 9-(biphenyl-4-yl)-10-phenylphenanthrene (3ac) ${ }^{[3]}$ in $71 \%$ yield $(28.9 \mathrm{mg})$ as white solid.

\section{Procedure C: synthesis of 3ah}

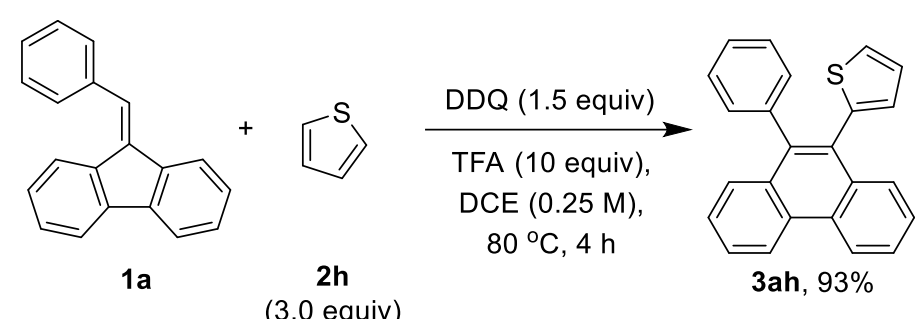

To a 1,2-dichloroethane (DCE) $(0.4 \mathrm{~mL})$ solution of 9-benzylidene-9H-fluorene (1a) $(25.4 \mathrm{mg}, 0.1 \mathrm{mmol})$ and thiophene $(\mathbf{2 h})(25.2 \mathrm{mg}$, $0.3 \mathrm{mmol}$ ) were added TFA (114 mg, $1 \mathrm{mmol}$ ) and 2,3-dichloro-5,6-dicyano-p-benzoquinone (DDQ) (33.8 mg, $0.15 \mathrm{mmol}$ ). The mixture in a $3 \mathrm{~mL}$ reaction vial was heated on an aluminum-block at $80^{\circ} \mathrm{C}$ for $4 \mathrm{~h}$. After cooling to room temperature, the resulting reaction mixture was quenched by pouring into saturated aqueous $\mathrm{NaHCO}_{3}(2 \mathrm{~mL})$. The organic layer was separated and the aqueous layer was extracted with dichloromethane $(2 \times 1 \mathrm{~mL})$. Combined organic layers were washed with water and brine, and dried over anhydrous $\mathrm{MgSO}_{4}$. After concentration, the resulting residue was purified by a short pad of silica gel using 1:10 mixture of DCM and hexane to afford the corresponding product 2-(10-phenylphenanthren-9-yl)thiophene (3ah $)^{[4]}$ in $93 \%$ yield (31.3 $\left.\mathrm{mg}\right)$ as white solid.

\subsection{General synthetic procedure of gram-scale synthesis}

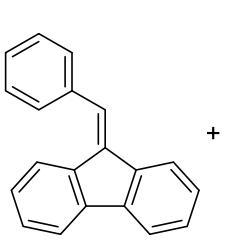

$1 \mathrm{a}$ $5 \mathrm{mmol}$<smiles>Brc1csc2ccccc12</smiles>

(3.0 equiv)
$\frac{\mathrm{DDQ}(1.5 \text { equiv })}{\text { TFA }(30 \mathrm{~mL}),}$ $80^{\circ} \mathrm{C}, 12 \mathrm{~h}$

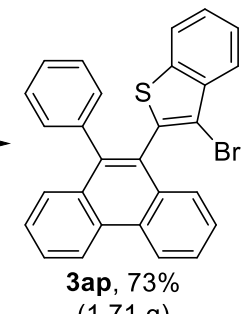

$(1.71 \mathrm{~g})$

To a $100 \mathrm{~mL}$ flask added 9-benzylidene-9H-fluorene (1a) (1271.7 mg, $5.0 \mathrm{mmol}$ ), 3-bromobenzo[b]thiophene (2p) (3196.4 mg, 15.0 $\mathrm{mmol}$ ) and trifluoroacetic acid (TFA) $(30.0 \mathrm{~mL})$, the resulted mixture was stirred for 5 min at room temperature, then 2,3-dichloro-5,6dicyano-p-benzoquinone (DDQ) $(1702.5 \mathrm{mg}, 7.5 \mathrm{mmol})$ was added. The mixture was heated in an oil bath at $80{ }^{\circ} \mathrm{C}$ for $12 \mathrm{~h}$. After cooling to room temperature, the resulting reaction mixture was quenched by aqueous $\mathrm{NaOH}(2 \mathrm{M}, 30 \mathrm{~mL})$. The organic layer was separated and the aqueous layer was extracted with dichloromethane $(2 \times 30 \mathrm{~mL})$. Combined organic layers were washed with water and brine, and dried over anhydrous $\mathrm{MgSO}_{4}$. After concentration, the resulting residue was purified by a short pad of silica gel using 1:10 mixture of DCM and hexane to afford the corresponding 3-bromo-2-(10-phenylphenanthren-9-yl)benzo[b]thiophene (3ap) in 73\% yield $(1710.0 \mathrm{mg})$ as white solid.

\subsection{General synthetic procedure of intramolecular $\mathrm{C}-\mathrm{H} / \mathrm{C}-\mathrm{Br}$ coupling}

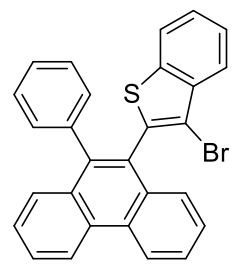

3ap

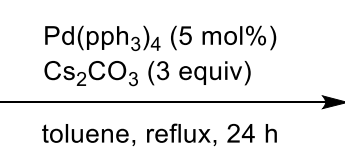

toluene, reflux, $24 \mathrm{~h}$

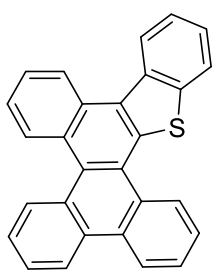

4ap, $84 \%$ 
A mixture of $3 \mathrm{ap}$ (93.1 mg, $0.2 \mathrm{mmol}), \mathrm{Cs}_{2} \mathrm{CO}_{3}(195.5 \mathrm{mg}, 0.6 \mathrm{mmol})$, and $\mathrm{Pd}\left(\mathrm{PPh}_{3}\right)_{4}(11.6 \mathrm{mg}, 0.01 \mathrm{mmol})$ was dissolved in degassed toluene $(5 \mathrm{~mL})$ under a $\mathrm{N}_{2}$ atmosphere in a $20 \mathrm{~mL}$ flask. After stirring at room temperature for 5 minutes, the mixture was refluxed for $24 \mathrm{~h}$ under $\mathrm{N}_{2}$ atmosphere. After completion, the mixture was extracted with $\mathrm{CH}_{2} \mathrm{Cl}_{2}$ and the organic layer was dried over $\mathrm{MgSO}_{4}$. After concentration of solvent, the residue was purified by a short pad of silica gel using 1:10 mixture of DCM and hexane, affording 4 ap in $84 \%$ yield $(64.6 \mathrm{mg})$ as light yellow solid.

\subsection{General synthetic procedure of one-pot oxidation process}

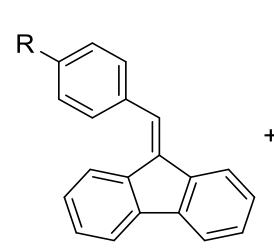

1a, $\mathrm{R}=\mathrm{H}$ 1f, $\mathrm{R}=\mathrm{OMe}$

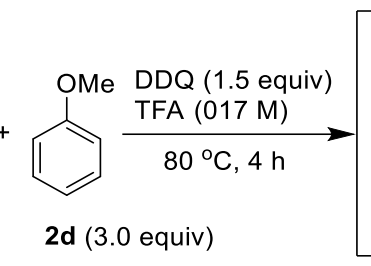

$0.1 \mathrm{mmol}$

To a trifluoroacetic acid (TFA) (0.6 mL) solution of 9-benzylidene-9H-fluorene (1a) (25.4 mg, $0.1 \mathrm{mmol})$ and anisole (2d) (32.4 mg, 0.3 mmol) was added 2,3-dichloro-5,6-dicyano-p-benzoquinone (DDQ) (33.8 mg, $0.15 \mathrm{mmol}$ ). The mixture in a $3 \mathrm{~mL}$ reaction vial was heated on an aluminum-block at $80^{\circ} \mathrm{C}$ for $4 \mathrm{~h}$. After cooling to room temperature, the resulting reaction mixture was transfered to a 50 $\mathrm{mL}$ flask and diluted with anhydrous dichloromethane $(9 \mathrm{~mL})$. To this solution, methanesulfonic acid (1 $\mathrm{mL})$ and $\mathrm{DDQ}$ (22.7 $\mathrm{mg}, 0.1$ $\mathrm{mmol})$ were added and the resulting mixture was stirred for $12 \mathrm{~h}$. The reaction was quenched by pouring onto saturated aqueous $\mathrm{NaHCO}_{3}(20 \mathrm{~mL})$. The organic layer was separated and the aqueous layer was extracted with dichloromethane $(2 \times 10 \mathrm{~mL}) . \mathrm{Combined}$ organic layers were washed with water and brine, and dried over anhydrous $\mathrm{MgSO}_{4}$. After concentration, the residue was purified by a short pad of silica gel using 1:10 mixture of DCM and hexane, affording $4 \mathrm{ad}^{[5]}$ in $93 \%$ overall yield (33.3 mg) as a white solid.

\subsection{General synthetic procedure of two-fold tandem oxidation to access 5 ah}

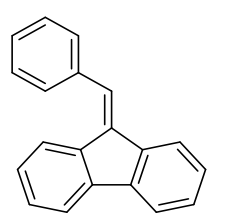

1a (3.0 equiv)

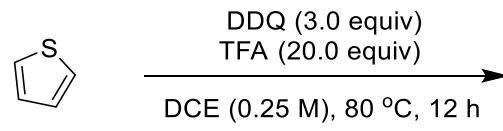

$2 \mathrm{~h}$

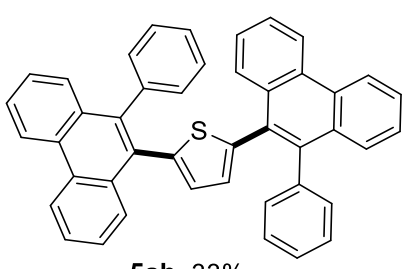

5ah, $33 \%$

To a solution of 9-benzylidene-9H-fluorene (1a) $(76.2 \mathrm{mg}, 0.3 \mathrm{mmol})$ and thiophene $(\mathbf{2 h})(8.4 \mathrm{mg}, 0.1 \mathrm{mmol})$ in 1,2-dichloroethane (DCE) $(0.4 \mathrm{~mL})$ were added TFA (228 mg, $2 \mathrm{mmol}$ ) and 2,3-dichloro-5,6-dicyano-p-benzoquinone (DDQ) (67.6 mg, $0.3 \mathrm{mmol})$. The mixture in a $3 \mathrm{~mL}$ reaction vial was heated on an aluminum-block at $80^{\circ} \mathrm{C}$ for $12 \mathrm{~h}$. After cooling to room temperature, the resulting reaction mixture was quenched by pouring into saturated aqueous $\mathrm{NaHCO}_{3}(2 \mathrm{~mL})$. The organic layer was separated and the aqueous layer was extracted with dichloromethane $(2 \times 1 \mathrm{~mL})$. Combined organic layers were washed with water and brine, and dried over anhydrous $\mathrm{MgSO}_{4}$. After concentration, the resulting residue was purified by a short pad of silica gel using 1:5 mixture of DCM and hexane to afford the corresponding product 2,5-bis(10-phenylphenanthren-9-yl)thiophene (5ah) in 33\% yield (19.4 mg) as yellow solid.

\subsection{General synthetic procedure of two-fold tandem oxidation to access $5 \mathrm{lj}$}




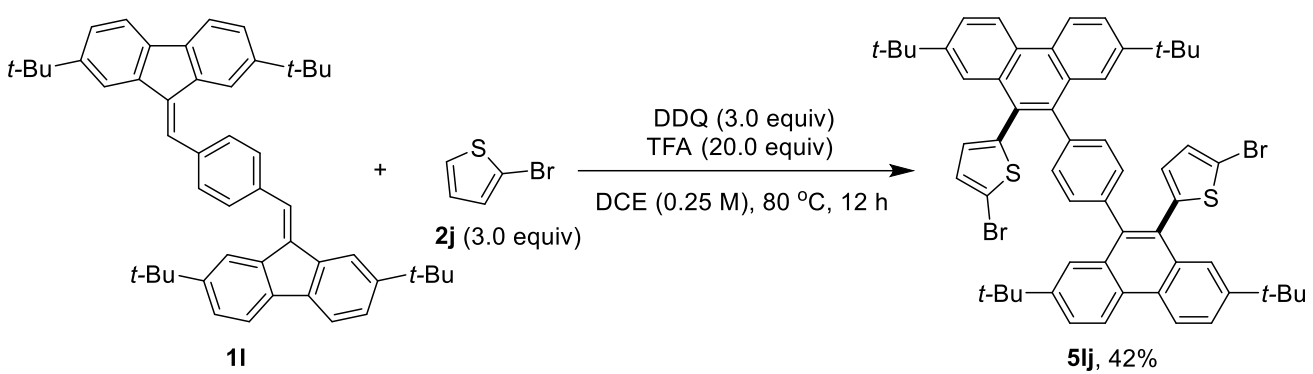

To a solution of 1,4-bis((2,7-di-tert-butyl-9H-fluoren-9-ylidene)methyl)benzene (1a) $(65.5 \mathrm{mg}, 0.1 \mathrm{mmol})$ and 2-bromothiophene (2h) (48.9 mg, $0.1 \mathrm{mmol})$ in 1,2-dichloroethane (DCE) $(0.4 \mathrm{~mL})$ were added TFA (228 mg, $2 \mathrm{mmol}$ ) and 2,3-dichloro-5,6-dicyano-pbenzoquinone (DDQ) $(67.6 \mathrm{mg}, 0.3 \mathrm{mmol})$. The mixture in a $3 \mathrm{~mL}$ reaction vial was heated on an aluminum-block at $80{ }^{\circ} \mathrm{C}$ for $12 \mathrm{~h}$. After cooling to room temperature, the resulting reaction mixture was quenched by pouring into saturated aqueous $\mathrm{NaHCO}_{3}(2 \mathrm{~mL})$. The organic layer was separated and the aqueous layer was extracted with dichloromethane $(2 \times 1 \mathrm{~mL})$. Combined organic layers were washed with water and brine, and dried over anhydrous $\mathrm{MgSO}_{4}$. After concentration, the resulting residue was purified by filtration through a short pad of silica gel using 1:5 mixture of DCM and hexane to afford the corresponding product 1,4-bis(10-(5-bromothiophen2-yl)-2,7-di-tert-butylphenanthren-9-yl)benzene (5lj) in $42 \%$ yield $(41.0 \mathrm{mg})$ as yellow solid.

\section{Control experiments for mechanistic study}

\section{(1) Control experiments using 3an'}

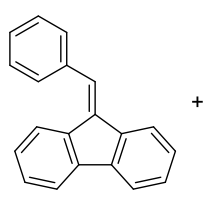

$1 \mathrm{a}$

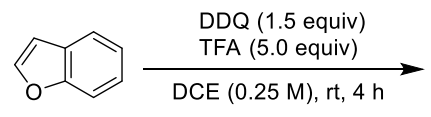

2n (3.0 equiv)

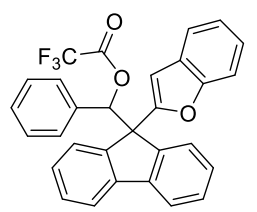

3an', $68 \%$

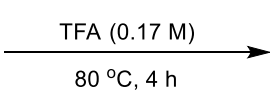

To a 1,2-dichloroethane (DCE) $(0.4 \mathrm{~mL})$ solution of 9-benzylidene-9H-fluorene (1a) $(25.4 \mathrm{mg}, 0.1 \mathrm{mmol})$, and benzofuran $(\mathbf{2 n})$ (35.4 $\mathrm{mg}, 0.3 \mathrm{mmol}$ ) were added TFA ( $57.0 \mathrm{mg}, 0.5 \mathrm{mmol}$ ) and 2,3-dichloro-5,6-dicyano-p-benzoquinone (DDQ) (33.8 mg, $0.15 \mathrm{mmol})$. The mixture in a $3 \mathrm{~mL}$ reaction vial was stirred at room temperature for $4 \mathrm{~h}$. After completion, the resulting reaction mixture was quenched by pouring into saturated aqueous $\mathrm{NaHCO}_{3}(2 \mathrm{~mL})$. The organic layer was separated and the aqueous layer was extracted with dichloromethane $(2 \times 1 \mathrm{~mL})$. Combined organic layers were washed with water and brine, and dried over anhydrous $\mathrm{MgSO}_{4}$. After concentration, the resulting residue was purified by a short pad of silica gel using 1:10 mixture of DCM and hexane to afford the corresponding product (9-(benzofuran-2-yl)-9H-fluoren-9-yl)(phenyl)methyl 2,2,2-trifluoroacetate (3an') in 68\% yield (32.9 mg) as white solid.

The mixture of 3an' (48.4 mg, $0.1 \mathrm{mmol})$ and TFA $(0.6 \mathrm{~mL})$ in a $3 \mathrm{~mL}$ reaction vial was heated on an aluminum-block at $80{ }^{\circ} \mathrm{C}$ for $4 \mathrm{~h}$. After cooling to room temperature, the resulting reaction mixture was quenched by pouring into saturated aqueous $\mathrm{NaHCO}_{3}(2 \mathrm{~mL})$. The organic layer was separated and the aqueous layer was extracted with dichloromethane $(2 \times 1 \mathrm{~mL})$. The combined organic layer was washed with water and brine, and dried over anhydrous $\mathrm{MgSO}_{4}$. After concentration, the resulting residue was purified by a short pad of silica gel using 1:5 mixture of DCM and hexane to afford the corresponding product 2-(10-phenylphenanthren-9-yl)benzofuran (3an) in $90 \%$ yield $(33.3 \mathrm{mg}$ ) as white solid.

(2) Reaction of $1 \mathbf{a}$ with $\mathbf{2 n}$ under the standard conditions in the presence of radical scavenger 


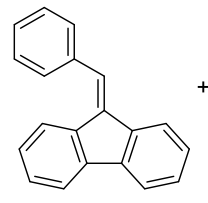

$1 \mathrm{a}$

(0.1 $\mathrm{mmol})$

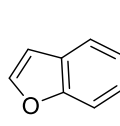

2n

(3 equiv)

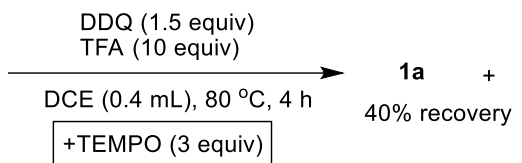

+ TEMPO ( 3 equiv)

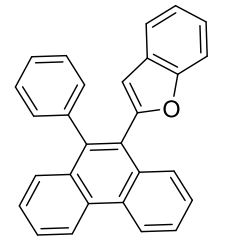

$3 a n$

not detected

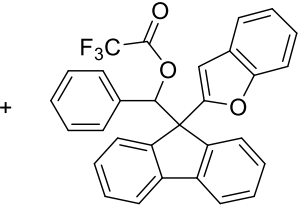

3an', 10\%

To a 1,2-dichloroethane (DCE) $(0.4 \mathrm{~mL})$ of 9-benzylidene-9H-fluorene (1a) (25.4 mg, $0.1 \mathrm{mmol})$, and benzofuran (2n) (35.4 mg, 0.3 $\mathrm{mmol}$ ) were added TFA (57.0 mg, $0.5 \mathrm{mmol}$ ), 2,3-dichloro-5,6-dicyano-p-benzoquinone (DDQ) (33.8 mg, 0.15 mmol), and (2,2,6,6tetramethylpiperidin-1-yl)oxyl (TEMPO) $(46.9 \mathrm{mg}, 0.3 \mathrm{mmol})$. The mixture in a $3 \mathrm{~mL}$ reaction vial was stirred at room temperature for 4 h. After completion, the resulting reaction mixture was quenched by pouring into saturated aqueous $\mathrm{NaHCO}_{3}(2 \mathrm{~mL})$. The organic layer was separated and the aqueous layer was extracted with dichloromethane $(2 \times 1 \mathrm{~mL})$. The combined organic layer was washed with water and brine, and dried over anhydrous $\mathrm{MgSO}_{4}$. After concentration, the resulting residue was analyzed by ${ }^{1} \mathrm{H}$ NMR spctrum using $\mathrm{CH}_{2} \mathrm{Br}_{2}$ as an internal standard. As aresult, no desired product 3an was detected and the byproduct 3an' was obtained in $10 \%$ yield together with the recovered $1 \mathrm{a}(40 \%)$, indicating that the desired tandem oxidation was completely hampered by a radical scavenger. This result suggested that the present reaction involves the formation of radical species.

\section{Proposed Mechanism}

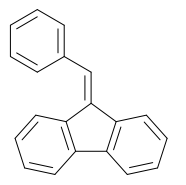

$1 \mathrm{a}$

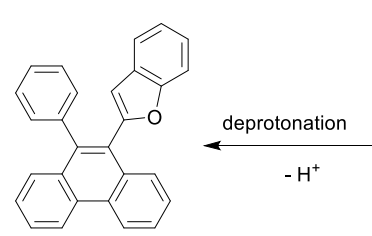

3an

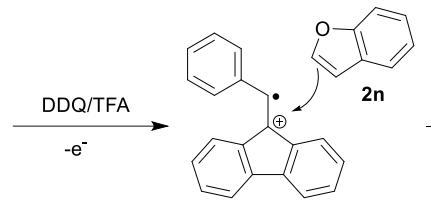

radical cation $\mathbf{A}$
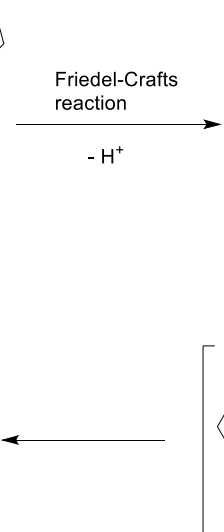

cation D $(-19.6 \mathrm{kcal} / \mathrm{mol})$

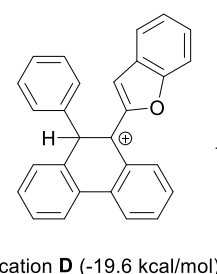

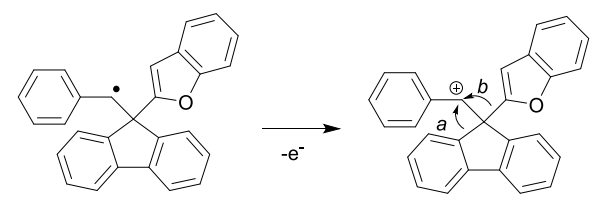

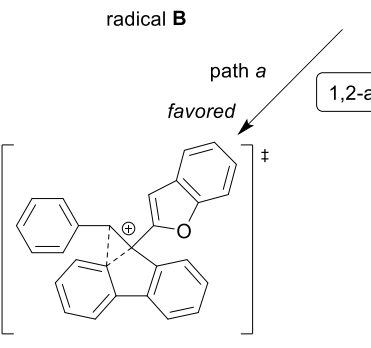
cation $\mathbf{C}(0.0 \mathrm{kcal} / \mathrm{mol})$ path $b$
disfavored

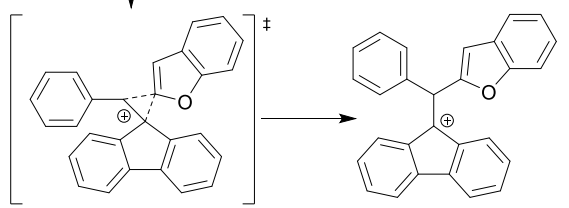

TS2 $(6.9 \mathrm{kcal} / \mathrm{mol})$

\section{X-Ray crystallographic analysis of 3an'}

The single crystal of 3an' was prepared by slow evaperation of a ethanol solution of compound 3an' at room temperature. 


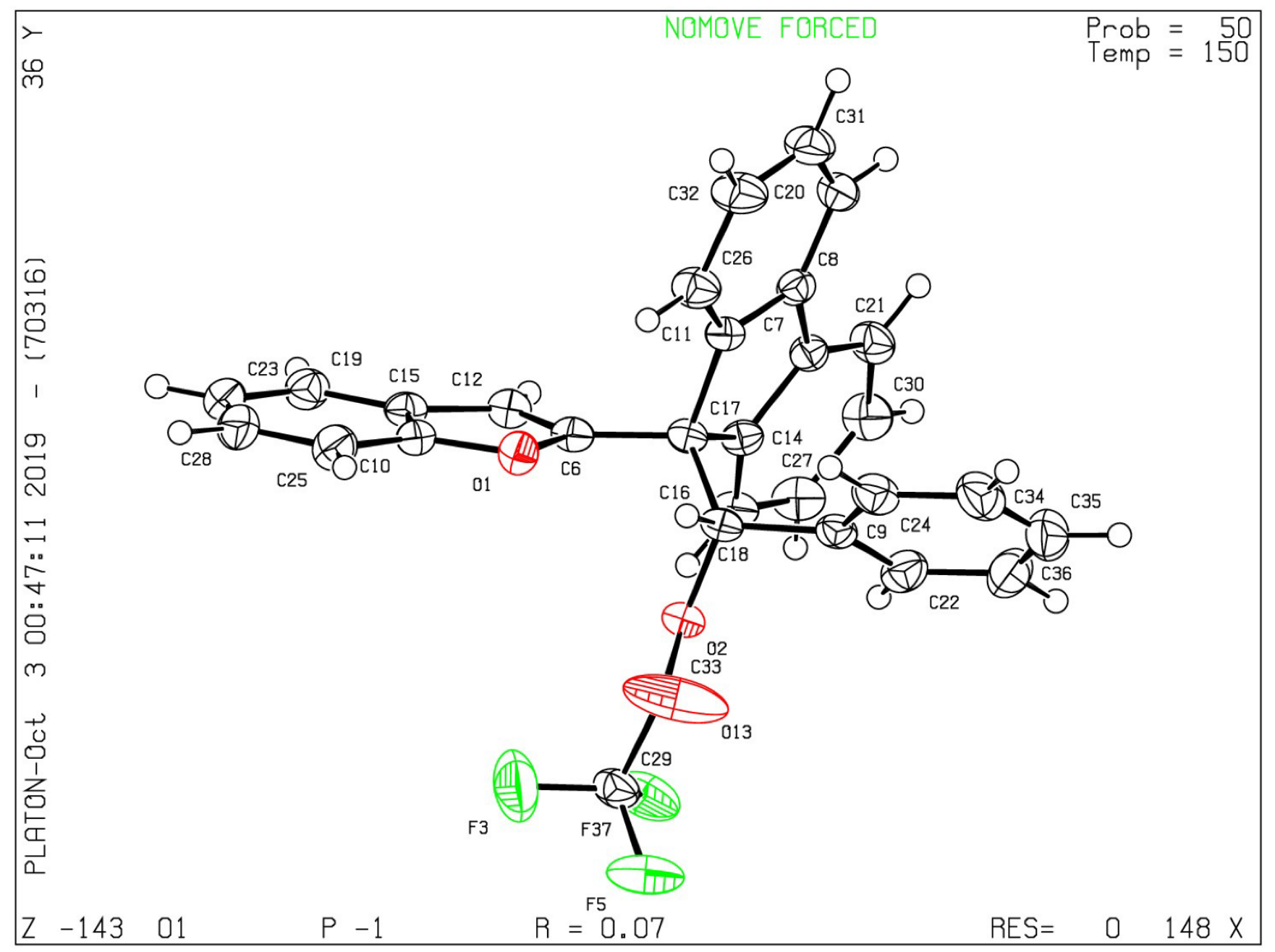

Figure S1. ORTEP drawing of 3an'. Thermal ellipsoids are shown at 50\% probability.

\section{Data Collection}

A colorless prism crystal of $\mathrm{C}_{30} \mathrm{H}_{19} \mathrm{~F}_{3} \mathrm{O}_{3}$ having approximate dimensions of $0.300 \times 0.200 \times 0.200 \mathrm{~mm}$ was mounted on a glass fiber. All measurements were made on a Rigaku XtaLAB mini diffractometer using graphite monochromated Mo-K $\alpha$ radiation.

The crystal-to-detector distance was $50.00 \mathrm{~mm}$.

Cell constants and an orientation matrix for data collection corresponded to a primitive triclinic cell with dimensions:

$$
\begin{array}{ll}
\mathrm{a}=8.887(2) \AA & \alpha=96.273(7)^{\mathrm{O}} \\
\mathrm{b}=11.324(3) \AA & \beta=105.606(7)^{\mathrm{O}} \\
\mathrm{c}=12.938(3) \AA & \gamma=106.641(8)^{\mathrm{O}} \\
\mathrm{V}=1176.8(5) \AA^{3} &
\end{array}
$$

For $\mathrm{Z}=2$ and F.W. $=484.47$, the calculated density is $1.367 \mathrm{~g} / \mathrm{cm}^{3}$. Based on a statistical analysis of intensity distribution, and the successful solution and refinement of the structure, the space group was determined to be:

$$
\text { P-1 (\#2) }
$$

The data were collected at a temperature of $-123 \pm 1^{\circ} \mathrm{C}$ to a maximum $2 \theta$ value of $55.0^{\circ}$. A total of 540 oscillation images were collected. A sweep of data was done using $\omega$ scans from -60.0 to $120.0^{\circ}$ in $1.00^{\circ}$ step, at $\chi=54.0^{\circ}$ and $\phi=0.0^{\circ}$. The exposure rate was 24.0 [sec. $/^{\circ}$ ]. The detector swing angle was $30.00^{\circ}$. A second sweep was performed using $\omega$ scans from -60.0 to $120.0^{\circ}$ in $1.00^{\circ}$ step, at $\chi=54.0^{\circ}$ and $\phi=120.0^{\circ}$. The exposure rate was $24.0\left[\mathrm{sec} . /^{\circ}\right]$. The detector swing angle was $30.00^{\mathrm{o}}$. Another sweep was performed using $\omega$ scans from -60.0 to $120.0^{\circ}$ in $1.00^{\mathrm{O}}$ step, at $\chi=54.0^{\circ}$ and $\phi=240.0^{\circ}$. The exposure rate was $24.0\left[\mathrm{sec} . /^{\circ}\right.$ ]. The detector swing angle was $30.00^{\circ}$. Another sweep was performed using $\omega$ scans from -60.0 to $120.0^{\circ}$ in $1.00^{\circ}$ step, at $\chi=54.0^{\circ}$ and $\phi=0.0^{\circ}$. The exposure rate was $24.0\left[\mathrm{sec} . /^{\circ}\right.$ ]. The detector swing angle was $30.00^{\circ}$. Another sweep was performed using $\omega$ scans from -60.0 to $120.0^{\circ}$ in $1.00^{\circ}$ step, at $\chi=54.0^{\circ}$ and $\phi=120.0^{\circ}$. The exposure rate was 24.0 [sec. $/^{\circ}$ ]. The detector 
swing angle was $30.00^{\circ}$. Another sweep was performed using $\omega$ scans from -60.0 to $120.0^{\circ}$ in $1.00^{\circ}$ step, at $\chi=54.0^{\circ}$ and $\phi=240.0^{\circ}$. The exposure rate was $24.0\left[\mathrm{sec} . /^{\mathrm{O}}\right.$ ]. The detector swing angle was $30.00^{\circ}$. The crystal-to-detector distance was $50.00 \mathrm{~mm}$. Readout was performed in the 0.073 mm pixel mode.

\section{Data Reduction}

Of the 12459 reflections were collected, where 5384 were unique $\left(\mathrm{R}_{\mathrm{int}}=0.1034\right)$. Data were collected and processed using CrystalClear (Rigaku). 1

The linear absorption coefficient, $\mu$, for Mo-K $\alpha$ radiation is $1.033 \mathrm{~cm}^{-1}$. An empirical absorption correction was applied which resulted in transmission factors ranging from 0.637 to 0.980 . The data were corrected for Lorentz and polarization effects.

$\underline{\text { Structure Solution and Refinement }}$

The structure was solved by direct methods ${ }^{2}$ and expanded using Fourier techniques. The non-hydrogen atoms were refined anisotropically. Hydrogen atoms were refined using the riding model. The final cycle of full-matrix least-squares refinement ${ }^{3}$ on $\mathrm{F}^{2}$ was based on 5384 observed reflections and 325 variable parameters and converged (largest parameter shift was 0.00 times its esd) with unweighted and weighted agreement factors of:

$$
\begin{gathered}
\mathrm{R} 1=\mathrm{S}\|\mathrm{Fo}|-| \mathrm{Fc}\| / \mathrm{S}|\mathrm{Fo}|=0.0744 \\
\mathrm{wR} 2=\left[\mathrm{S}\left(\mathrm{w}\left(\mathrm{Fo}^{2}-\mathrm{Fc}^{2}\right)^{2}\right) / \mathrm{S} w\left(\mathrm{Fo}^{2}\right)^{2}\right]^{1 / 2}=0.2106
\end{gathered}
$$

The goodness of $\mathrm{fit}^{4}$ was 1.03 . Unit weights were used. The maximum and minimum peaks on the final difference Fourier map corresponded to 0.50 and $-0.38 \mathrm{e}^{-} / \AA^{3}$, respectively.

Neutral atom scattering factors were taken from International Tables for Crystallography (IT), Vol. C, Table 6.1.1.4 5. Anomalous dispersion effects were included in Fcalc ${ }^{6}$; the values for Df' and Df" were those of Creagh and McAuley ${ }^{7}$. The values for the mass attenuation coefficients are those of Creagh and Hubbell ${ }^{8}$. All calculations were performed using the CrystalStructure ${ }^{9}$ crystallographic software package except for refinement, which was performed using SHELXL Version $2013 / 4^{10}$.

\section{References}

(1) CrystalClear: Data Collection and Processing Software, Rigaku Corporation (1998-2015). Tokyo 196-8666, Japan.

(2) SHELXT Version 2014/4: Sheldrick, G. M. (2014). Acta Cryst. A70, C1437.

(3) Least Squares function minimized: (SHELXL Version 2013/4)

$$
\mathrm{S} w\left(\mathrm{~F}_{\mathrm{o}}{ }^{2}-\mathrm{F}_{\mathrm{C}}{ }^{2}\right)^{2} \quad \text { where } \mathrm{w}=\text { Least Squares weights. }
$$

(4) Goodness of fit is defined as:

$$
\begin{aligned}
& {\left[\mathrm{Sw}\left(\mathrm{F}_{\mathrm{O}}{ }^{2}-\mathrm{F}_{\mathrm{C}}{ }^{2}\right)^{2} /\left(\mathrm{N}_{\mathrm{O}}-\mathrm{N}_{\mathrm{V}}\right)\right]^{1 / 2}} \\
& \text { where: } \quad \mathrm{N}_{\mathrm{O}}=\text { number of observations } \\
& \mathrm{N}_{\mathrm{V}}=\text { number of variables }
\end{aligned}
$$

(5) International Tables for Crystallography, Vol.C (1992). Ed. A.J.C. Wilson, Kluwer Academic Publishers, Dordrecht, Netherlands, Table 6.1.1.4, pp. 572.

(6) Ibers, J. A. \& Hamilton, W. C.; Acta Crystallogr., 17, 781 (1964).

(7) Creagh, D. C. \& McAuley, W.J .; "International Tables for Crystallography", Vol C, (A.J.C. Wilson, ed.), Kluwer Academic Publishers, Boston, Table 4.2.6.8, pages 219-222 (1992).

(8) Creagh, D. C. \& Hubbell, J.H..; "International Tables for Crystallography", Vol C, (A.J.C. Wilson, ed.), Kluwer Academic Publishers, Boston, Table 4.2.4.3, pages 200-206 (1992).

(9) CrystalStructure 4.3: Crystal Structure Analysis Package, Rigaku Corporation (2000-2019). Tokyo 196-8666, Japan.

(10) SHELXL Version 2013/4: Sheldrick, G. M. (2008). Acta Cryst. A64, 112-122. 
EXPERIMENTAL DETAILS

A. Crystal Data

Empirical Formula

Formula Weight

Crystal Color, Habit

Crystal Dimensions

Crystal System

Lattice Type

Lattice Parameters

Space Group

$Z$ value

$\mathrm{D}_{\text {calc }}$

$F_{000}$

m(MoKa)

Diffractometer

Radiation

Voltage, Current

Temperature

Detector Aperture

Data Images

w oscillation Range $(c=54.0, f=0.0)$

Exposure Rate

Detector Swing Angle

w oscillation Range $(\mathrm{c}=54.0, \mathrm{f}=120.0)$

Exposure Rate

Detector Swing Angle

w oscillation Range $(c=54.0, f=240.0)$

Exposure Rate

Detector Swing Angle
$\mathrm{C}_{30} \mathrm{H}_{19} \mathrm{~F}_{3} \mathrm{O}_{3}$

484.47

colorless, prism

$0.300 \times 0.200 \times 0.200 \mathrm{~mm}$

triclinic

Primitive

$a=8.887(2) \AA$

$\mathrm{b}=11.324(3) \AA$

$\mathrm{c}=12.938(3) \AA$

$\mathrm{a}=96.273(7)^{\circ}$

$\mathrm{b}=105.606(7)^{\circ}$

$g=106.641(8)^{0}$

$V=1176.8(5) \AA^{3}$

P-1 (\#2)

2

$1.367 \mathrm{~g} / \mathrm{cm}^{3}$

500.00

$1.033 \mathrm{~cm}^{-1}$

B. Intensity Measurements

XtaLAB mini

$\operatorname{MoKa}(I=0.71075 \AA)$

graphite monochromated

$50 \mathrm{kV}, 12 \mathrm{~mA}$

$-123.0^{\circ} \mathrm{C}$

$75.0 \mathrm{~mm}$ (diameter)

540 exposures

$-60.0-120.0^{\circ}$

$24.0 \mathrm{sec} . / 0$

$30.00^{\circ}$

$-60.0-120.0^{0}$

$24.0 \mathrm{sec} . /^{\circ}$

$30.00^{\circ}$

$-60.0-120.0^{\circ}$

$24.0 \mathrm{sec} . / 0$

$30.00^{\circ}$ 
w oscillation Range $(c=54.0, f=0.0)$

Exposure Rate

Detector Swing Angle

w oscillation Range $(c=54.0, f=120.0)$

Exposure Rate

Detector Swing Angle

w oscillation Range $(\mathrm{c}=54.0, \mathrm{f}=240.0)$

Exposure Rate

Detector Swing Angle

Detector Position

Pixel Size

$2 q_{\max }$

No. of Reflections Measured

Corrections

Structure Solution

Refinement

Function Minimized

Least Squares Weights

$2 q_{\max }$ cutoff

Anomalous Dispersion

No. Observations (All reflections)

No. Variables

Reflection/Parameter Ratio

Residuals: R1 (I>2.00s(I))

Residuals: $\mathrm{R}$ (All reflections)

Residuals: wR2 (All reflections)

Goodness of Fit Indicator

Max Shift/Error in Final Cycle

Maximum peak in Final Diff. Map

Minimum peak in Final Diff. Map $-60.0-120.0^{\circ}$

$24.0 \mathrm{sec} . / 0$

$30.00^{\circ}$

$-60.0-120.0^{\circ}$

$24.0 \mathrm{sec} . / 0$

$30.00^{\circ}$

$-60.0-120.0^{\circ}$

$24.0 \mathrm{sec} . / 0$

$30.00^{\circ}$

$50.00 \mathrm{~mm}$

$0.073 \mathrm{~mm}$

$55.0^{\circ}$

Total: 12459

Unique: $5384\left(R_{\text {int }}=0.1034\right)$

Lorentz-polarization

Absorption

(trans. factors: $0.637-0.980$ )

C. Structure Solution and Refinement

Direct Methods (SHELXT Version 2014/4)

Full-matrix least-squares on $\mathrm{F}^{2}$

$S w\left(F o^{2}-F c^{2}\right)^{2}$

$w=1 /\left[s^{2}\left(F^{2}\right)+(0.0916 \cdot P)^{2}\right.$

$+0.3007 \cdot \mathrm{P}]$

where $\mathrm{P}=\left(\operatorname{Max}\left(\mathrm{Fo}^{2}, 0\right)+2 \mathrm{Fc}^{2}\right) / 3$

$55.0^{\circ}$

All non-hydrogen atoms

5384

325

16.57

0.0744

0.0903

0.2106

1.032

0.000

$0.50 \mathrm{e}^{-} / \AA^{3}$

$-0.38 \mathrm{e}^{-} / \AA^{3}$ 


\section{Analytical data of starting substrates and products}

\section{9-benzylidene-9H-fluorene $(1 \mathrm{a})^{[6]}$}<smiles>C(=C1c2ccccc2-c2ccccc21)c1ccccc1</smiles>

Purified by flash column chromatography (silica gel), [Hexane /DCM = 10:1 (v/v)] to give 1a as a white solid $(2.70 \mathrm{mmol}, 686.7 \mathrm{mg}$, 90\% yield); ${ }^{1} \mathrm{H}$ NMR $\left(400 \mathrm{MHz}, \mathrm{CDCl}_{3}\right) \delta 7.81(\mathrm{~d}, \mathrm{~J}=6.9 \mathrm{~Hz}, 1 \mathrm{H}), 7.72-7.75(\mathrm{~m}, 3 \mathrm{H}), 7.58-7.63(\mathrm{~m}, 3 \mathrm{H}), 7.47-7.51(\mathrm{~m}, 2 \mathrm{H}), 7.32-7.44$ $(\mathrm{m}, 4 \mathrm{H}), 7.07-7.11(\mathrm{~m}, 1 \mathrm{H}) ;{ }^{13} \mathrm{C} \mathrm{NMR}\left(100 \mathrm{MHz}, \mathrm{CDCl}_{3}\right) \delta 141.2,139.4,139.1,136.9,136.5,136.4,129.2,128.5,128.2,128.0,127.3$, $127.0,126.6,124.4,120.2,119.7,119.6$, one $\mathrm{sp}^{2}$ carbon peak was not shown due to superimposition.

\section{9-(2-bromo-5-methylbenzylidene)-9H-fluorene (1b) ${ }^{[7]}$}<smiles>Cc1ccc(Br)c(C=C2c3ccccc3-c3ccccc32)c1</smiles>

Purified by flash column chromatography (silica gel), [Hexane /DCM = 10:1 (v/v)] to give $\mathbf{1 b}$ as a light yellow solid $(1.70 \mathrm{mmol}, 590.2$ mg, 85\% yield); ${ }^{1} \mathrm{H}$ NMR $\left(400 \mathrm{MHz}, \mathrm{CDCl}_{3}\right) \delta 7.85(\mathrm{~d}, \mathrm{~J}=6.9 \mathrm{~Hz}, 1 \mathrm{H}), 7.72-7.74(\mathrm{~m}, 2 \mathrm{H}), 7.56-7.61(\mathrm{~m}, 2 \mathrm{H}), 7.49(\mathrm{~s}, 1 \mathrm{H}), 7.32-7.43(\mathrm{~m}$, $4 \mathrm{H}), 7.05-7.11(\mathrm{~m}, 2 \mathrm{H}), 2.35$ (s, 3H); ${ }^{13} \mathrm{C} \mathrm{NMR}\left(100 \mathrm{MHz}, \mathrm{CDCl}_{3}\right)$ ठ 141.3, 139.3, 139.1, 137.1, 137.0, 136.9, 136.4, 132.6, 131.9, 130.5, $128.66,128.4,127.1,126.7,126.1,124.4,120.6,119.8,119.6,20.8$, one $\mathrm{sp}^{2}$ carbon peak was not shown due to superimposition.

\section{9-(4-fluorobenzylidene)-9H-fluorene (1c)}<smiles>Fc1ccc(C=C2c3ccccc3-c3ccccc32)cc1</smiles>

Purified by flash column chromatography (silica gel), [Hexane /DCM =10:1 (v/v)] to give 1c as a light yellow solid (1.66 mmol, 452.1 mg, 83\% yield); ${ }^{1} \mathrm{H}$ NMR (600 MHz, $\left.\mathrm{CDCl}_{3}\right) \delta 7.79$ (q, J = 3.4 Hz, 1H), $7.75(\mathrm{t}, J=6.9 \mathrm{~Hz}, 2 \mathrm{H}), 7.63(\mathrm{~s}, 1 \mathrm{H}), 7.54-7.58(\mathrm{~m}, 3 \mathrm{H}), 7.42(\mathrm{q}$ $J=7.3 \mathrm{~Hz}, 1 \mathrm{H}), 7.36(\mathrm{~m}, 2 \mathrm{H}), 7.16-7.20(\mathrm{~m}, 2 \mathrm{H}), 7.12(\mathrm{q}, J=8.0 \mathrm{~Hz}, 1 \mathrm{H}) ;{ }^{13} \mathrm{C} \mathrm{NMR}\left(150 \mathrm{MHz}, \mathrm{CDCl}_{3}\right) \delta 162.4\left(\mathrm{~d}, J_{\mathrm{C}-\mathrm{F}}=247.1 \mathrm{~Hz}\right)$, 141.3, 139.3, 139.1, 136.6, 136.3, 132.7, 131.0 (d, JC-F = 8.7 Hz), 128.6, 128.2, 127.0, 126.7, 126.0, 124.2, 120.2, 119.8, 119.6, 115.5 (d, $J_{C-F}=20.2 \mathrm{~Hz}$ ); HRMS (FD+): [m/z]: calcd. for $\mathrm{C}_{20} \mathrm{H}_{13} \mathrm{~F}, 272.1001$; found 272.1000 .

\section{9-(4-(trifluoromethyl)benzylidene)-9H-fluorene (1d) ${ }^{[8]}$}<smiles>FC(F)(F)c1ccc(C=C2c3ccccc3-c3ccccc32)cc1</smiles>

Purified by flash column chromatography (silica gel), [Hexane /DCM = 10:1 (v/v)] to give $1 \mathrm{~d}$ as a light yellow solid (1.64 mmol, 528.6 $\mathrm{mg}, 82 \%$ yield); ${ }^{1} \mathrm{H}$ NMR $\left(600 \mathrm{MHz}, \mathrm{CDCl}_{3}\right) \delta 7.74(\mathrm{~m}, 7 \mathrm{H}), 7.61(\mathrm{~s}, 1 \mathrm{H}), 7.36-7.50(\mathrm{~m}, 4 \mathrm{H}), 7.10-7.12(\mathrm{~m}, 1 \mathrm{H}) ;{ }^{13} \mathrm{C} \mathrm{NMR}(150 \mathrm{MHz}$, $\left.\mathrm{CDCl}_{3}\right) \delta 141.5,140.6,139.3,139.0,137.8,136.1,129.7$ (q, $\left.J_{\mathrm{C}-\mathrm{F}}=33.2 \mathrm{~Hz}\right), 129.5,129.0,128.7,127.1,126.8,125.4\left(\mathrm{q}, J_{\mathrm{C}-\mathrm{F}}=2.9 \mathrm{~Hz}\right.$ ), 125.0, 124.3, 124.2 (q, JC-F $=271.7 \mathrm{~Hz}), 120.4,119.9,119.7$.

\section{9-(3-nitrobenzylidene)-9H-fluorene (1e)}


<smiles>O=[N+]([O-])c1cccc(C=C2c3ccccc3-c3ccccc32)c1</smiles>

Purified by flash column chromatography (silica gel), [Hexane /DCM = 10:1 to $2: 1(\mathrm{v} / \mathrm{v})$ ] to give $1 \mathrm{e}$ as a light yellow solid (1.20 mmol, $359.2 \mathrm{mg}, 60 \%$ yield); ${ }^{1} \mathrm{H}$ NMR $\left(600 \mathrm{MHz}, \mathrm{CDCl}_{3}\right) \delta 8.42(\mathrm{~s}, 1 \mathrm{H}), 8.24(\mathrm{~d}, J=8.2 \mathrm{~Hz}, 1 \mathrm{H}), 7.89(\mathrm{~d}, \mathrm{~J}=7.6 \mathrm{~Hz}, 1 \mathrm{H}), 7.76(\mathrm{~d}, \mathrm{~J}=7.6 \mathrm{~Hz}$, $1 \mathrm{H}), 7.70(\mathrm{~d}, \mathrm{~J}=7.6 \mathrm{~Hz}, 2 \mathrm{H}), 7.60(\mathrm{t}, J=7.9 \mathrm{~Hz}, 1 \mathrm{H}), 7.53-7.56(\mathrm{~m}, 1 \mathrm{H}), 7.40(\mathrm{t}, J=7.6 \mathrm{~Hz}, 1 \mathrm{H}), 7.33-7.37(\mathrm{~m}, 3 \mathrm{H}), 7.06(\mathrm{t}, J=7.2 \mathrm{~Hz}$, $1 \mathrm{H}) ;{ }^{13} \mathrm{C} \mathrm{NMR}\left(150 \mathrm{MHz}, \mathrm{CDCl}_{3}\right) \delta 148.3,141.6,139.4,138.8,138.6,138.5,135.7,135.3,129.4,129.3,128.9,127.2,126.9,124.1$, 124.0, 123.5, 122.6, 120.4, 120.0, 119.7; HRMS (FD+): [m/z]: calcd. for $\mathrm{C}_{20} \mathrm{H}_{13} \mathrm{NO}_{2}$, 299.0946; found 299.0945.

9-(4-methoxybenzylidene)-9H-fluorene (1f) ${ }^{[9]}$

$\mathrm{MeO}$<smiles>Cc1ccc(C=C2c3ccccc3-c3ccccc32)cc1</smiles>

Purified by flash column chromatography (silica gel), [Hexane /DCM =10:1 to $5: 1(\mathrm{v} / \mathrm{v})$ ] to give $1 \mathrm{e}$ as a yellow solid (1.74 mmol, 494.8 mg, 87\% yield); ${ }^{1} \mathrm{H}$ NMR $\left(400 \mathrm{MHz}, \mathrm{CDCl}_{3}\right) \delta 7.79(\mathrm{~d}, J=7.3 \mathrm{~Hz}, 1 \mathrm{H}), 7.72-7.75(\mathrm{~m}, 3 \mathrm{H}), 7.67(\mathrm{~s}, 1 \mathrm{H}), 7.57(\mathrm{~d}, J=8.7 \mathrm{~Hz}, 2 \mathrm{H}), 7.31-$ $7.40(\mathrm{~m}, 3 \mathrm{H}), 7.11(\mathrm{t}, J=7.6 \mathrm{~Hz}, 1 \mathrm{H}), 7.00(\mathrm{~d}, J=8.7 \mathrm{~Hz}, 2 \mathrm{H}), 3.90(\mathrm{~s}, 3 \mathrm{H}) ;{ }^{13} \mathrm{C} \mathrm{NMR}\left(100 \mathrm{MHz}, \mathrm{CDCl}_{3}\right) \delta 159.5,141.1,139.6,138.9$, $136.58,135.4,130.8,129.0,128.3,127.9,127.3,126.9,126.6,124.1,120.1,119.7,119.5,113.9,55.3$.

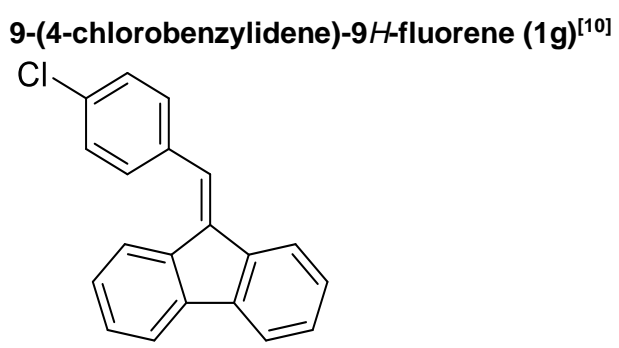

Purified by flash column chromatography (silica gel), [Hexane /DCM = 10:1 (v/v)] to give $\mathbf{1 g}$ as a yellow solid $(1.54 \mathrm{mmol}, 444.7 \mathrm{mg}$, $77 \%$ yield); ${ }^{1} \mathrm{H}$ NMR $\left(400 \mathrm{MHz}, \mathrm{CDCl}_{3}\right) \delta 7.77$ (d, $\left.J=7.3 \mathrm{~Hz}, 1 \mathrm{H}\right), 7.72(\mathrm{~d}, J=7.3 \mathrm{~Hz}, 2 \mathrm{H}), 7.59(\mathrm{~s}, 1 \mathrm{H}), 7.53(\mathrm{t}, J=7.3 \mathrm{~Hz}, 3 \mathrm{H}), 7.32-$ $7.45(\mathrm{~m}, 5 \mathrm{H}), 7.09(\mathrm{t}, J=7.6 \mathrm{~Hz}, 1 \mathrm{H}) ;{ }^{13} \mathrm{C} \mathrm{NMR}\left(100 \mathrm{MHz}, \mathrm{CDCl}_{3}\right) \delta 141.3,139.2,139.2,137.0,136.2,135.3,133.8,130.6,128.8$, $128.4,127.0,126.7,125.6,124.3,120.2,119.8,119.6$, one $\mathrm{sp}^{2}$ carbon peak was not shown due to superimposition.

\section{9-benzylidene-2,7-di-tert-butyl-9H-fluorene (1h)}<smiles>CC(C)(C)c1ccc2c(c1)C(=Cc1ccccc1)c1cc(C(C)(C)C)ccc1-2</smiles>

Purified by flash column chromatography (silica gel), [Hexane /DCM = 10:1 (v/v)] to give $\mathbf{1 h}$ as a yellow solid $(1.56 \mathrm{mmol}, 571.8 \mathrm{mg}$, 78\% yield); ${ }^{1} \mathrm{H}$ NMR $\left(400 \mathrm{MHz}, \mathrm{CDCl}_{3}\right)$ ס 7.78-7.81 (m, 1H), 7.67-7.70 (m, 1H), 7.55-7.62 (m, 4H), 7.52 (s, $\left.1 \mathrm{H}\right), 7.46(\mathrm{t}, J=6.9 \mathrm{~Hz}, 2 \mathrm{H})$, $7.40(\mathrm{~d}, J=7.3 \mathrm{~Hz}, 2 \mathrm{H}), 7.30(\mathrm{~d}, J=8.2 \mathrm{~Hz}, 1 \mathrm{H}), 1.41(\mathrm{~s}, 9 \mathrm{H}), 1.15(\mathrm{~s}, 9 \mathrm{H}) ;{ }^{13} \mathrm{C} \mathrm{NMR}\left(100 \mathrm{MHz}, \mathrm{CDCl}_{3}\right) \delta$ 149.7, 149.2, 139.4, 138.7, 137.3, 136.8, 136.8, 129.3, 128.4, 127.8, 126.0, 125.5, 121.7, 118.9, 118.8 (7), 116.8 (9), 35.0, 34.7, 31.6, 31.3, two sp ${ }^{2}$ carbon peaks were not shown due to superimposition, HRMS (FD+): [m/z]: calcd. for $\mathrm{C}_{28} \mathrm{H}_{30}, 366.2348$; found 366.2348.

\section{9-benzylidene-2,7-dibromo-9H-fluorene (1i)}


<smiles>Brc1ccc2c(c1)C(=Cc1ccccc1)c1cc(Br)ccc1-2</smiles>

Purified by flash column chromatography (silica gel), [Hexane $/ \mathrm{DCM}=10: 1(\mathrm{v} / \mathrm{v})$ ] to give $1 \mathrm{i}$ as a yellow solid $(1.52 \mathrm{mmol}, 626.4 \mathrm{mg}$, $76 \%$ yield);; ${ }^{1} \mathrm{H}$ NMR $\left(400 \mathrm{MHz}, \mathrm{CDCl}_{3}\right) \delta 7.80$ (d, $\left.J=0.9 \mathrm{~Hz}, 1 \mathrm{H}\right), 7.68$ (d, $\left.J=1.4 \mathrm{~Hz}, 1 \mathrm{H}\right), 7.59(\mathrm{~s}, 1 \mathrm{H}), 7.37-7.53(\mathrm{~m}, 9 \mathrm{H}) ;{ }^{13} \mathrm{C} \mathrm{NMR}$ $\left(100 \mathrm{MHz}, \mathrm{CDCl}_{3}\right) \delta 140.9,138.9,137.8,136.7,135.5,134.2,131.3,131.0,129.8,129.1,128.7,128.6,127.2,123.4,121.1,120.8$, 120.7, 120.6; two $\mathrm{sp}^{2}$ carbon peaks are not shown due to superimposition. HRMS (FD+): [m/z]: calcd. for $\mathrm{C}_{20} \mathrm{H}_{12} \mathrm{Br}_{2}, 409.9306$; found 409.9306 .

\section{9-benzylidene-2,7-dichloro-9H-fluorene (1j)}<smiles>Clc1ccc2c(c1)C(=Cc1ccccc1)c1cc(Cl)ccc1-2</smiles>

Purified by flash column chromatography (silica gel), [Hexane $/ \mathrm{DCM}=10: 1(\mathrm{v} / \mathrm{v})$ ] to give $1 \mathrm{j}$ as a yellow solid $(1.48 \mathrm{mmol}, 478.4 \mathrm{mg}$, $74 \%$ yield); ${ }^{1} \mathrm{H}$ NMR $\left(400 \mathrm{MHz}, \mathrm{CDCl}_{3}\right) \delta 7.71(\mathrm{~d}, J=1.8 \mathrm{~Hz}, 1 \mathrm{H}), 7.68(\mathrm{~s}, 1 \mathrm{H}), 7.42-7.57(\mathrm{~m}, 8 \mathrm{H}), 7.33(\mathrm{dd}, J=7.8,1.8 \mathrm{~Hz}, 1 \mathrm{H}), 7.25-$ $7.28(\mathrm{~m}, 1 \mathrm{H}) ;{ }^{13} \mathrm{C}$ NMR $\left(100 \mathrm{MHz}, \mathrm{CDCl}_{3}\right) \delta 140.9,138.6,137.7,136.5,135.6,134.5,133.0,132.5,129.8,129.1,128.7,128.69,128.6$,

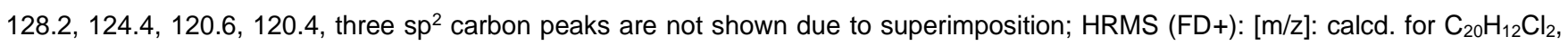
322.0316 ; found 322.0316 .

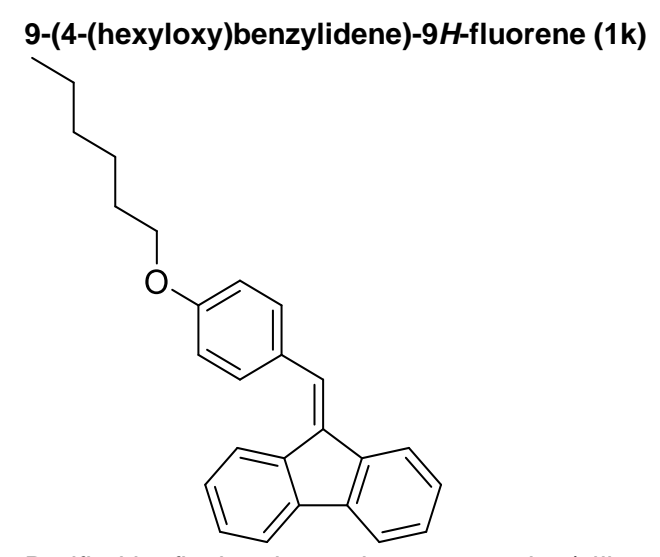

Purified by flash column chromatography (silica gel), [Hexane /DCM =10:1 (v/v)] to give $1 \mathbf{k}$ as an orange solid $(1.42 \mathrm{mmol}, 503.4 \mathrm{mg}$, $71 \%$ yield); ${ }^{1} \mathrm{H}$ NMR $\left(600 \mathrm{MHz}, \mathrm{CDCl}_{3}\right) \delta 7.74-7.80(\mathrm{~m}, 4 \mathrm{H}), 7.67(\mathrm{~s}, 1 \mathrm{H}), 7.56(\mathrm{~d}, J=8.9 \mathrm{~Hz}, 2 \mathrm{H}), 7.32-7.40(\mathrm{~m}, 3 \mathrm{H}), 7.11(\mathrm{~m}, 1 \mathrm{H})$, 6.99-7.00 (m, 2H), $4.04(\mathrm{t}, J=6.5 \mathrm{~Hz}, 2 \mathrm{H}), 1.85(\mathrm{~m}, 2 \mathrm{H}), 1.50-1.55(\mathrm{~m}, 2 \mathrm{H}), 1.39-1.42(\mathrm{~m}, 4 \mathrm{H}), 0.95-0.97(\mathrm{~m}, 3 \mathrm{H}) ;{ }^{13} \mathrm{C} \mathrm{NMR}(150 \mathrm{MHz}$, $\left.\mathrm{CDCl}_{3}\right) \delta 159.1,141.1,139.7,138.9,136.6,135.3,130.8,128.8,128.2,127.8,127.5,126.9,126.6,124.2,120.0,119.7,119.5,114.4$, 68.1, 31.6, 29.3, 25.8, 22.6, 14.1; HRMS (FD+): [m/z]: calcd. for $\mathrm{C}_{26} \mathrm{H}_{26} \mathrm{O}$, 354.1984; found 354.1984.

\section{1,4-bis((2,7-di-tert-butyl-9H-fluoren-9-ylidene)methyl)benzene (1I)}<smiles>CC(C)(C)c1ccc2c(c1)C(=Cc1ccc(C=C3c4cc(C(C)(C)C)ccc4-c4ccc(C(C)(C)C)cc43)cc1)c1cc(C(C)(C)C)ccc1-2</smiles>

Purified by flash column chromatography (silica gel), [Hexane /DCM = 10:1 (v/v)] to give 11 as a yellow solid $(1.22 \mathrm{mmol}, 799.1 \mathrm{mg}$, $61 \%$ yield); ${ }^{1} \mathrm{H}$ NMR (600 MHz, $\left.\mathrm{CDCl}_{3}\right)$ $\delta$ 7.85-7.86 (m, 4H), $7.77(\mathrm{~s}, 4 \mathrm{H}), 7.73(\mathrm{~s}, 2 \mathrm{H}), 7.61$ (dd, $\left.J=7.9,6.5 \mathrm{~Hz}, 4 \mathrm{H}\right), 7.43(\mathrm{dd}, J=7.9$, 
$1.7 \mathrm{~Hz}, 2 \mathrm{H}), 7.35$ (dd, $J=7.9,1.7 \mathrm{~Hz}, 2 \mathrm{H}), 1.45$ (s, 18H), 1.20 (s, 18H); ${ }^{13} \mathrm{C} \mathrm{NMR}\left(150 \mathrm{MHz}, \mathrm{CDCl}_{3}\right) \delta$ 149.8, 149.4, 139.5, 138.8, 137.4, $136.9,136.8,136.7,129.5,125.8,125.7,125.6,121.7,119.0,116.9,35.0,34.8,31.6,31.4$; one sp ${ }^{2}$ carbon peak was not shown due to superimposition. HRMS (FD+): [m/z]: calcd. for $\mathrm{C}_{50} \mathrm{H}_{54}, 654.4226$; found 654.4226 .

\section{9-(3,4-dimethylphenyl)-10-phenylphenanthrene (3aa)}

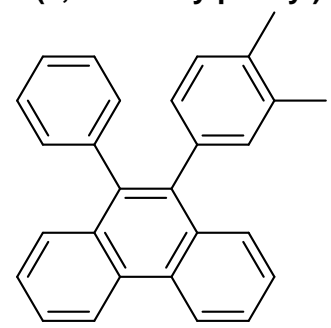

Purified by flash column chromatography (silica gel), [Hexane /DCM = 10:1 (v/v)] to give 3aa as a white solid $(0.095 \mathrm{mmol}, 34.1 \mathrm{mg}$, 95\% yield); ${ }^{1} \mathrm{H}$ NMR (400 MHz, $\mathrm{CDCl}_{3}$ ) $\delta 8.81$ (d, $\left.J=8.2 \mathrm{~Hz}, 2 \mathrm{H}\right), 7.60-7.68(\mathrm{~m}, 3 \mathrm{H}), 7.46-7.55(\mathrm{~m}, 3 \mathrm{H}), 7.16-7.28(\mathrm{~m}, 5 \mathrm{H}), 6.87-7.00$ $(\mathrm{m}, 3 \mathrm{H}), 2.22(\mathrm{~s}, 3 \mathrm{H}), 2.16(\mathrm{~s}, 3 \mathrm{H}) ;{ }^{13} \mathrm{C} N M R\left(100 \mathrm{MHz}, \mathrm{CDCl}_{3}\right) \delta 139.8,137.3,137.0,136.8,135.5,134.4,132.2,132.1,132.0,131.0$ (1), 131.0, 129.9, 129.6, 128.8, 128.4, 128.0, 127.8, 127.5, 126.5, 126.3, 126.2 (5), 126.2, 122.4, 122.4, 19.7, 19.5. HRMS (MALDI): [m/z]: calcd. for $\mathrm{C}_{28} \mathrm{H}_{22}, 358.1716$; found 370.1716 .

\section{9-phenyl-10-(p-tolyl)phenanthrene (3ab) $)^{[11]}$}

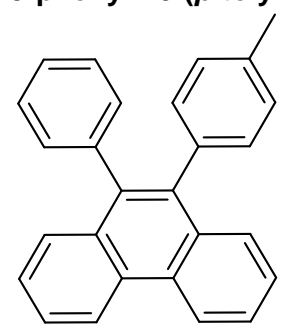

Purified by flash column chromatography (silica gel), [Hexane $/ \mathrm{DCM}=10: 1(\mathrm{v} / \mathrm{v})$ ] to give $3 \mathrm{ab}$ as a white solid $(0.06 \mathrm{mmol}, 20.7 \mathrm{mg}$, $60 \%$ yield); ${ }^{1} \mathrm{H}$ NMR $\left(400 \mathrm{MHz}, \mathrm{CDCl}_{3}\right) \delta 8.81(\mathrm{~d}, J=8.2 \mathrm{~Hz}, 2 \mathrm{H}), 7.66(\mathrm{t}, J=7.6 \mathrm{~Hz}, 2 \mathrm{H}), 7.54-7.60(\mathrm{~m}, 2 \mathrm{H}), 7.48(\mathrm{t}, J=7.1 \mathrm{~Hz}, 2 \mathrm{H})$, 7.16-7.28 (m, 5H), 7.02-7.08 (m , 4H), $2.32(\mathrm{~s}, 3 \mathrm{H}) ;{ }^{13} \mathrm{C} \mathrm{NMR}\left(100 \mathrm{MHz}, \mathrm{CDCl}_{3}\right) \delta$ 139.7, 137.2, 137.1, 136.4, 135.9, 132.1, 131.9, $131.0,130.8,130.0,129.9,128.3,127.9,127.8,127.6,126.5,126.4,126.3,126.3,122.4,21.2$; two sp ${ }^{2}$ carbon peaks are not shown due to superimposition.

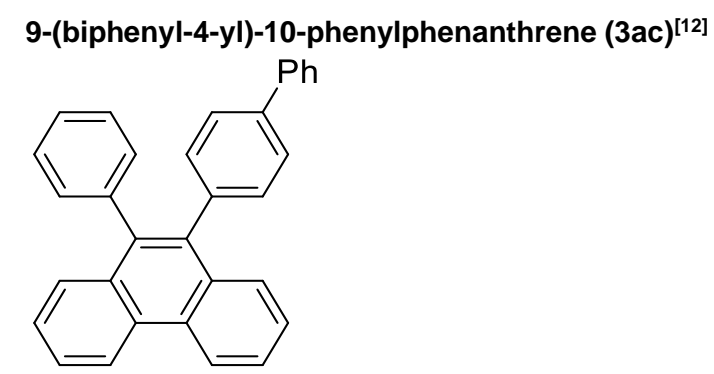

Purified by flash column chromatography (silica gel), [Hexane /DCM = 10:1 (v/v)] to give 3ac as a white solid $(0.071 \mathrm{mmol}, 28.9 \mathrm{mg}$, $71 \%$ yield); ${ }^{1} \mathrm{H}$ NMR $\left(400 \mathrm{MHz}, \mathrm{CDCl}_{3}\right) \delta 8.82(\mathrm{~d}, J=8.2 \mathrm{~Hz}, 2 \mathrm{H}), 7.64-7.70(\mathrm{~m}, 3 \mathrm{H}), 7.57-7.61(\mathrm{~m}, 3 \mathrm{H}), 7.48-7.53(\mathrm{~m}, 4 \mathrm{H}), 7.41-7.46$ $(\mathrm{m}, 2 \mathrm{H}), 7.31-7.35(\mathrm{~m}, 1 \mathrm{H}), 7.17-7.27(\mathrm{~m}, 7 \mathrm{H}) ;{ }^{13} \mathrm{C} \mathrm{NMR}\left(100 \mathrm{MHz}, \mathrm{CDCl}_{3}\right) \delta 140.7,139.5,138.9,138.6,137.3,136.8,131.9,131.8$, 131.5, 131.0, 130.0, 128.7, 127.8 (4), 127.8 (2), 127.6, 127.2, 126.9, 126.6 (4), 126.6 (2), 126.5, 126.4, 126.2, 122.5, 122.5, two sp 2 carbon peaks are not shown due to superimposition.

\section{9-(4-methoxyphenyl)-10-phenylphenanthrene (3ad) ${ }^{[13]}$}<smiles>COc1ccc(-c2c(-c3ccccc3)c3ccccc3c3ccccc23)cc1</smiles> 
Purified by flash column chromatography (silica gel), [Hexane /DCM = 10:1 (v/v)] to give 3ad as a white solid $(0.099 \mathrm{mmol}, 35.7 \mathrm{mg}$, $99 \%$ yield); ${ }^{1} \mathrm{H}$ NMR (400 MHz, $\left.\mathrm{CDCl}_{3}\right) \delta 8.82(\mathrm{~d}, \mathrm{~J}=8.2 \mathrm{~Hz}, 2 \mathrm{H}), 7.48-7.69(\mathrm{~m}, 6 \mathrm{H}), 7.07-7.30(\mathrm{~m}, 7 \mathrm{H}), 6.80(\mathrm{~m}, 2 \mathrm{H}), 3.79(\mathrm{~s}, 3 \mathrm{H}) ;{ }^{13} \mathrm{C}$ NMR $\left(100 \mathrm{MHz}, \mathrm{CDCl}_{3}\right) \delta 158.0,139.7,137.4,136.8,132.2,132.0,131.9,131.7,131.0,130.0,129.9,127.8,127.8,127.6,126.5$, $126.4,126.3(0), 126.2(7), 122.4,113.0,55.1$, two $\mathrm{sp}^{2}$ carbon peaks are not shown due to superimposition.

\section{5-(10-phenylphenanthren-9-yl)benzo[d][1,3]dioxole (3ae)}

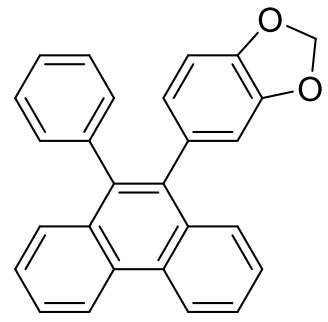

Purified by flash column chromatography (silica gel), [Hexane /DCM =10:1 (v/v)] to give 3ae as a white solid $(0.082 \mathrm{mmol}, 30.7 \mathrm{mg}$, $82 \%$ yield); ${ }^{1} \mathrm{H}-N M R 8.80$ (d, J = 8.2 Hz, 2H), 7.62-7.69 (m, 3H), 7.46-7.55 (m, 3H), 7.24-7.31 (m, 3H), 7.15-7.19 (m, 2H), 6.70 (d, J = $7.8 \mathrm{~Hz}, 1 \mathrm{H}), 6.66(\mathrm{~d}, \mathrm{~J}=1.4 \mathrm{~Hz}, 1 \mathrm{H}), 6.61(\mathrm{dd}, \mathrm{J}=7.8,1.8 \mathrm{~Hz}, 1 \mathrm{H}), 5.95(\mathrm{~d}, \mathrm{~J}=1.4 \mathrm{~Hz}, 1 \mathrm{H}), 5.92(\mathrm{~d}, \mathrm{~J}=1.4 \mathrm{~Hz}, 1 \mathrm{H}) ;{ }^{13} \mathrm{C} \mathrm{NMR}(100$ $\left.\mathrm{MHz}, \mathrm{CDCl}_{3}\right) \delta 146.9,146.0,139.6,137.4,136.6,133.2,132.0,131.9,131.0,130.8,130.0,129.9,127.8,127.7,127.6,126.6(1), 126.5$ (9), 126.5, 126.4, 124.5, 122.5, 111.5, 107.7, 100.8, one sp² carbon peak was not shown due to superimposition. HRMS (FD+): [m/z]: calcd. for $\mathrm{C}_{27} \mathrm{H}_{18} \mathrm{O}_{2}, 374.1306$; found 374.1308 .

\section{9-(naphthalen-1-yl)-10-phenylphenanthrene (3af)}

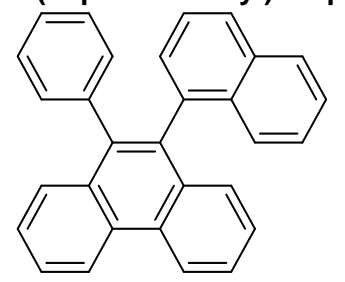

Purified by flash column chromatography (silica gel), [Hexane $/ \mathrm{DCM}=10: 1(\mathrm{v} / \mathrm{v})$ ] to give 3af as a white solid $(0.071 \mathrm{mmol}, 27.0 \mathrm{mg}$, $71 \%$ yield); ${ }^{1} \mathrm{H}$ NMR $\left(400 \mathrm{MHz}, \mathrm{CDCl}_{3}\right) \delta 8.93(\mathrm{~d}, \mathrm{~J}=8.2 \mathrm{~Hz}, 2 \mathrm{H}), 7.88-7.91(\mathrm{~m}, 1 \mathrm{H}), 7.64-7.83(\mathrm{~m}, 7 \mathrm{H}), 7.53-7.63(\mathrm{~m}, 4 \mathrm{H}), 7.42(\mathrm{~d}, \mathrm{~J}$ $=8.2 \mathrm{~Hz}, 1 \mathrm{H}), 7.24-7.35(\mathrm{~m}, 4 \mathrm{H}), 7.18-7.23(\mathrm{~m}, 1 \mathrm{H}) ;{ }^{13} \mathrm{C} \mathrm{NMR}\left(100 \mathrm{MHz}, \mathrm{CDCl}_{3}\right) \delta$ 139.4, 137.5, 137.1, $136.9,132.9,131.9(9), 131.9$ (5), 131.9, 131.1, 130.8, 130.0, 129.9 (6), 129.3, 127.9, 127.6, 127.1, 126.6, 126.5, 126.4 (4), 126.4 (1), 125.8, 125.7, 122.5, five sp² carbon peaks are not shown due to superimposition. HRMS (FD+): [m/z]: calcd. for $\mathrm{C}_{30} \mathrm{H}_{20}, 380.1565$; found 380.1564 .

\section{1-(10-phenylphenanthren-9-yl)dibenzo[ghi,mno]fluoranthene (3ag)}<smiles></smiles>

Purified by flash column chromatography (silica gel), [Hexane /DCM = 5:1 (v/v)] to give 3ag as a white solid $(0.015 \mathrm{mmol}, 7.5 \mathrm{mg}, 15 \%$ yield); ${ }^{1} \mathrm{H}$ NMR (400 MHz, $\left.\mathrm{CDCl}_{3}\right) \delta 8.88(\mathrm{~d}, J=8.2 \mathrm{~Hz}, 1 \mathrm{H}), 8.83(\mathrm{~d}, J=8.2 \mathrm{~Hz}, 1 \mathrm{H}), 7.81(\mathrm{~d}, J=8.2 \mathrm{~Hz}, 3 \mathrm{H}), 7.68-7.76(\mathrm{~m}, 4 \mathrm{H}), 7.57$ $(\mathrm{t}, J=8.5 \mathrm{~Hz}, 5 \mathrm{H}), 7.46(\mathrm{~d}, J=7.3 \mathrm{~Hz}, 1 \mathrm{H}), 7.29(\mathrm{~s}, 2 \mathrm{H}), 7.11(\mathrm{~m}, 4 \mathrm{H}), 6.82(\mathrm{~d}, J=7.8 \mathrm{~Hz}, 1 \mathrm{H}) ;{ }^{13} \mathrm{C} \mathrm{NMR}\left(100 \mathrm{MHz}, \mathrm{CDCl}_{3}\right) \delta 139.5$, 139.2, 138.7, 136.2, 135.7, 135.5, 135.1, 135.0, 134.4, 132.2, 132.0, 131.4, 131.3, 130.8, 130.7, 130.5, 130.3, 130.2 (8), 129.8, 128.7, 128.0, 127.9, 127.8, 127.0, 126.9 (7), 126.9, 126.8, 126.8, 126.7, 126.6, 126.4, 126.3 (8), 122.6, 122.4, HRMS (FD+): [m/z]: calcd. for $\mathrm{C}_{40} \mathrm{H}_{22}, 502.1721$; found 502.1721 .

\section{9-(2-bromo-5-methylphenyl)-10-(4-methoxyphenyl)phenanthrene (3bd)}




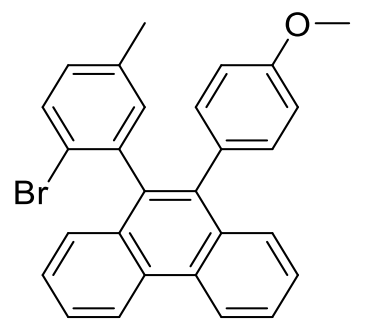

Purified by flash column chromatography (silica gel), [Hexane $/ \mathrm{DCM}=4: 1(\mathrm{v} / \mathrm{v})$ ] to give $3 \mathrm{bd}$ as a white solid $(0.085 \mathrm{mmol}, 38.5 \mathrm{mg}$, $85 \%$ yield); ${ }^{H} \mathrm{H}$ NMR (600 MHz, $\mathrm{CDCl}_{3}$ ) $\delta 8.81$ (d, J = 8.2 Hz, 2H), 7.66-7.69 (m, 2H), 7.60 (d, J = 8.2 Hz, 1H), 7.49-7.53 (m, 2H), 7.41$7.42(\mathrm{~m}, 1 \mathrm{H}), 7.38(\mathrm{~d}, \mathrm{~J}=8.2 \mathrm{~Hz}, 1 \mathrm{H}), 7.31(\mathrm{dd}, \mathrm{J}=8.2,2.1 \mathrm{~Hz}, 1 \mathrm{H}), 7.12(\mathrm{dd}, \mathrm{J}=8.6,2.4 \mathrm{~Hz}, 1 \mathrm{H}), 6.95(\mathrm{~d}, \mathrm{~J}=2.1 \mathrm{~Hz}, 1 \mathrm{H}), 6.91(\mathrm{~d}, \mathrm{~J}$ $=8.2 \mathrm{~Hz}, 1 \mathrm{H}), 6.83-6.85(\mathrm{~m}, 1 \mathrm{H}), 6.80(\mathrm{dd}, \mathrm{J}=8.9,2.7 \mathrm{~Hz}, 1 \mathrm{H}), 3.80(\mathrm{~s}, 3 \mathrm{H}), 2.21(\mathrm{~s}, 3 \mathrm{H}) ;{ }^{13} \mathrm{C} \mathrm{NMR}\left(100 \mathrm{MHz}, \mathrm{CDCl}_{3}\right) \delta 158.3,140.3$, 136.8, 136.6, 136.5, 133.4, 132.3, 131.8, 131.7, 131.5, 130.9, 130.7, 130.3, 129.8, 129.4, 127.9, 127.1, 126.8, 126.5, 126.4, 122.6, 122.5, 121.7, 113.0, 55.1, 20.8. HRMS (FD+): [m/z]: calcd. for $\mathrm{C}_{28} \mathrm{H}_{21} \mathrm{BrO}, 452.0775$; found 452.0773 .

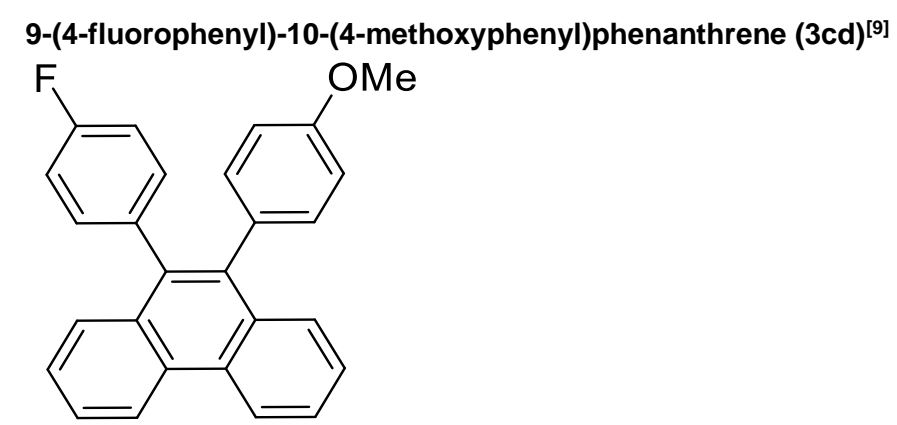

Purified by flash column chromatography (silica gel), [Hexane $/ \mathrm{DCM}=10: 1(\mathrm{v} / \mathrm{v})$ ] to give $3 \mathrm{~cd}$ as a white solid $(0.09 \mathrm{mmol}, 34.1 \mathrm{mg}$, $90 \%$ yield); ${ }^{1} \mathrm{H}-\mathrm{NMR}\left(600 \mathrm{MHz}, \mathrm{CDCl}_{3}\right) \delta 8.82(\mathrm{~d}, \mathrm{~J}=7.6 \mathrm{~Hz}, 2 \mathrm{H}), 7.69(\mathrm{td}, J=7.6,1.4 \mathrm{~Hz}, 2 \mathrm{H}), 7.63(\mathrm{~d}, J=8.2 \mathrm{~Hz}, 1 \mathrm{H}), 7.51-7.57(\mathrm{~m}$, $3 \mathrm{H}), 7.12(\mathrm{td}, J=6.0,2.7 \mathrm{~Hz}, 2 \mathrm{H}), 7.06(\mathrm{~m}, 2 \mathrm{H}), 6.96-6.99(\mathrm{~m}, 2 \mathrm{H}), 6.82(\mathrm{~d}, J=8.9 \mathrm{~Hz}, 2 \mathrm{H}), 3.82(\mathrm{~s}, 3 \mathrm{H}) ;{ }^{13} \mathrm{C}-\mathrm{NMR}(151 \mathrm{MHz}, \mathrm{CDCl} 3)$ $\delta 161.4\left(\mathrm{~d}, J_{\mathrm{C}-\mathrm{F}}=244.2 \mathrm{~Hz}\right), 158.1,137.3,136.4,135.6\left(\mathrm{~d}, J_{\mathrm{C}-\mathrm{F}}=2.9 \mathrm{~Hz}\right), 132.5\left(\mathrm{~d}, \mathrm{~J}_{\mathrm{C}-\mathrm{F}}=7.2 \mathrm{~Hz}\right), 132.1,131.9(4), 131.8(5), 131.6$, $130.0,129.9,127.9,127.5,126.6,126.5,126.4,122.5(2), 122.4(5), 114.6$ (d, J J-F $=21.7 \mathrm{~Hz}), 113.1,55.1$.

\section{9-(4-methoxyphenyl)-10-(4-(trifluoromethyl)phenyl)phenanthrene (3dd) $)^{[14]}$ \\ $\mathrm{F}_{3} \mathrm{C}$

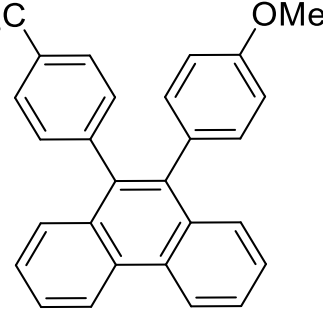

Purified by flash column chromatography (silica gel), [Hexane /DCM = 10:1 (v/v)] to give 3dd as a white solid $(0.071 \mathrm{mmol}, 30.4 \mathrm{mg}$, $71 \%$ yield); ${ }^{1} \mathrm{H}-\mathrm{NMR}\left(600 \mathrm{MHz}, \mathrm{CDCl}_{3}\right) \delta 8.83(\mathrm{dd}, J=8.2,2.8 \mathrm{~Hz}, 2 \mathrm{H}), 7.68-7.71(\mathrm{~m}, 2 \mathrm{H}), 7.63(\mathrm{dd}, J=8.2,1,5 \mathrm{~Hz}, 1 \mathrm{H}), 7.50-7.54(\mathrm{~m}$, $4 \mathrm{H}), 7.45$ (dd, $J=8.3,1.4 \mathrm{~Hz}, 1 \mathrm{H}), 7.29(\mathrm{~d}, J=8.2 \mathrm{~Hz}, 2 \mathrm{H}), 7.05(\mathrm{~d}, J=8.2 \mathrm{~Hz}, 2 \mathrm{H}), 6.81(\mathrm{~d}, J=8.2 \mathrm{~Hz}, 2 \mathrm{H}), 3.81(\mathrm{~s}, 3 \mathrm{H}) ;{ }^{13} \mathrm{C}-\mathrm{NMR}$ $\left(150 \mathrm{MHz}, \mathrm{CDCl}_{3}\right) \delta 158.3,143.8,137.1,135.9,132.0,131.9,131.4,131.3,131.1,130.2,130.0,128.7$ (q, JC-F = 30.8 Hz), 127.9, 127.3, $126.8,126.8,126.7,126.6,124.8\left(q, J_{C-F}=2.9 \mathrm{~Hz}\right), 124.1\left(q, J_{C-F}=270.1 \mathrm{~Hz}\right), 122.6,122.5,113.3,55.1$.

\section{9-(4-methoxyphenyl)-10-(3-nitrophenyl)phenanthrene (3ed)}

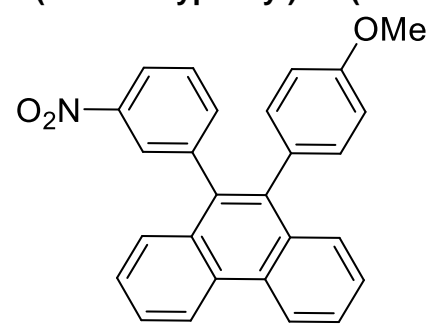

Purified by flash column chromatography (silica gel), [Hexane /DCM = 10:1 (v/v)] to give 3ed as a white solid $(0.041 \mathrm{mmol}, 16.6 \mathrm{mg}$, $41 \%$ yield); ${ }^{1} \mathrm{H}-\mathrm{NMR}\left(600 \mathrm{MHz}, \mathrm{CDCl}_{3}\right) \delta 9.17(\mathrm{dd}, J=8.2,1.4 \mathrm{~Hz}, 2 \mathrm{H}), 8.40-8.44(\mathrm{~m}, 2 \mathrm{H}), 8.03-8.06(\mathrm{~m}, 2 \mathrm{H}), 7.94(\mathrm{~d}, J=7.6 \mathrm{~Hz}, 1 \mathrm{H})$, 7.84-7.88 (m, 3H), 7.74-7.80 (m, 2H), 7.40-7.42 (m, 1H), 7.37 (dd, J = 8.6, 2.4 Hz, 1H), 7.12-7.15 (m, 2H), 4.12 (s, 3H); ${ }^{13} \mathrm{C}-\mathrm{NMR}(150$ 
$\left.\mathrm{MHz}, \mathrm{CDCl}_{3}\right) \delta 158.3,147.7,141.7,137.7,137.2,134.9,132.0,131.9,131.8,131.0,130.8,130.3,130.0,128.7,128.0,127.0,126.9$, 126.7, 126.0, 122.8, 122.5, 121.7, 113.6, 113.2, 55.1. HRMS (FD+): [m/z]: calcd. for $\mathrm{C}_{27} \mathrm{H}_{19} \mathrm{NO}, 405.1365$; found 405.1364 .

9,10-bis(4-methoxyphenyl)phenanthrene (3fd $)^{[10]}$

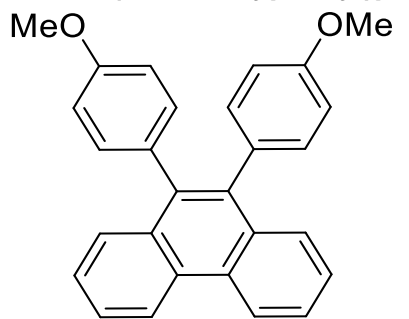

Purified by flash column chromatography (silica gel), [Hexane /DCM =10:1 (v/v)] to give $3 \mathrm{fd}$ as a white solid $(0.08 \mathrm{mmol}, 31.2 \mathrm{mg}, 80 \%$ yield); ${ }^{1} \mathrm{H}-\mathrm{NMR}\left(400 \mathrm{MHz}, \mathrm{CDCl}_{3}\right) \delta 8.80(\mathrm{~d}, \mathrm{~J}=8.2 \mathrm{~Hz}, 2 \mathrm{H}), 7.63-7.67(\mathrm{~m}, 2 \mathrm{H}), 7.59(\mathrm{~m}, 2 \mathrm{H}), 7.47-7.50(\mathrm{~m}, 2 \mathrm{H}), 7.06(\mathrm{dt}, J=9.2,2.4$ $\mathrm{Hz}, 4 \mathrm{H}), 6.79-6.82(\mathrm{~m}, 4 \mathrm{H}), 3.81(\mathrm{~s}, 6 \mathrm{H}) ;{ }^{13} \mathrm{C}-\mathrm{NMR}\left(100 \mathrm{MHz}, \mathrm{CDCl}_{3}\right) \delta$ 157.9, 137.1, 132.3, 132.0, 131.9 (5), 129.9, $127.8,126.5$, $126.2,122.4,113.1,55.1$.

\section{9-(4-chlorophenyl)-10-(3,4-dimethylphenyl)phenanthrene (3ga)}

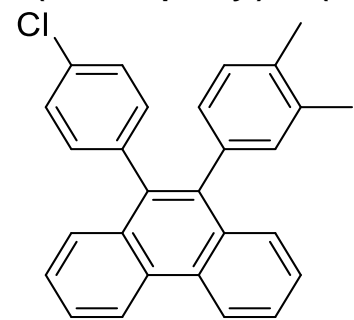

Purified by flash column chromatography (silica gel), [Hexane /DCM = 10:1 (v/v)] to give $3 \mathrm{ga}$ as a white solid $(0.091 \mathrm{mmol}, 35.8 \mathrm{mg}$, $91 \%$ yield); ${ }^{1} \mathrm{H}$ NMR (400 MHz, $\mathrm{CDCl}_{3}$ ) $\delta 8.80$ (dd, $\left.J=8.0,3.0 \mathrm{~Hz}, 2 \mathrm{H}\right), 7.60-7.68(\mathrm{~m}, 3 \mathrm{H}), 7.49-7.51$ (m, 3H), 7.23-7.25 (m, 2H), 7.13$7.09(\mathrm{~m}, 2 \mathrm{H}), 7.02(\mathrm{~d}, J=7.8 \mathrm{~Hz}, 1 \mathrm{H}), 6.92(\mathrm{~s}, 1 \mathrm{H}), 6.87$ (dd, $J=7.3,1.4 \mathrm{~Hz}, 1 \mathrm{H}), 2.26(\mathrm{~s}, 3 \mathrm{H}), 2.20(\mathrm{~s}, 3 \mathrm{H}) ;{ }^{13} \mathrm{C} \mathrm{NMR}(100 \mathrm{MHz}, \mathrm{CDCl})_{3}$ ठ 138.3, 137.6, 136.5, 135.7, 135.6 (8), 134.7, 132.4, 132.3, 132.0, 131.9 (9), 131.7, 130.0, 129.9, 129.0, 128.3, 128.0, 127.8 (3), 127.8 (0), 127.4, 126.6, 126.6, 126.5, 126.3, 122.5, 122.4, 19.7, 19.5, one $\mathrm{sp}^{2}$ carbon peak is not shown due to superimposition; HRMS (FD+): [m/z]: calcd. for $\mathrm{C}_{28} \mathrm{H}_{21} \mathrm{Cl}, 392.1332$; found 392.1330 .

\section{2,7-di-tert-butyl-9-(4-methoxyphenyl)-10-phenylphenanthrene (3hd)}

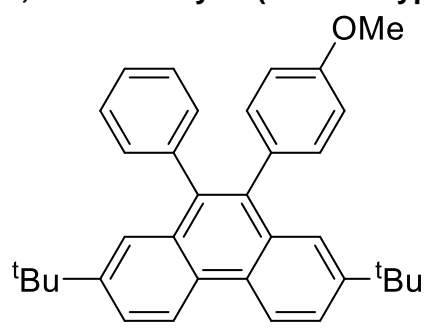

Purified by flash column chromatography (silica gel), [Hexane /DCM = 10:1 (v/v)] to give 3hd as a white solid $(0.083 \mathrm{mmol}, 39.2 \mathrm{mg}$, $83 \%$ yield); ${ }^{1} \mathrm{H}-\mathrm{NMR}\left(600 \mathrm{MHz}, \mathrm{CDCl}_{3}\right) \delta 8.70$ (d, $\left.J=8.2 \mathrm{~Hz}, 2 \mathrm{H}\right), 7.72(\mathrm{dq}, J=8.7,1.8 \mathrm{~Hz}, 2 \mathrm{H}), 7.59(\mathrm{~d}, J=2.3 \mathrm{~Hz}, 1 \mathrm{H}), 7.52(\mathrm{~d}, J=$ $1.8 \mathrm{~Hz}, 1 \mathrm{H}$ ), 7.15-7.28 (m, 5H), 7.08 (dt, $J=9.2,2.4 \mathrm{~Hz}, 2 \mathrm{H}), 6.79$ (dt, $J=9.2,2.4 \mathrm{~Hz}, 2 \mathrm{H}), 3.79(\mathrm{~s}, 3 \mathrm{H}), 1.30(\mathrm{~s}, 9 \mathrm{H}), 1.28(\mathrm{~s}, 9 \mathrm{H}) ;{ }^{13} \mathrm{C}-$ NMR $\left(150 \mathrm{MHz}, \mathrm{CDCl}_{3}\right) \delta 157.8,148.8,148.7,140.0,137.6,136.9,132.1$ (2), 132.0 (8), 131.7, 131.5, 131.1, 127.8, 127.7, 127.5, $126.2,124.3$ (8), 124.3 (6), 123.6, 123.5, 122.1, 112.9, 55.1, 34.8, 34.7 (7), 31.3, 31.2, one sp ${ }^{2}$ carbon peak is not shown due to superimposition; HRMS (FD+): [m/z]: calcd. for $\mathrm{C}_{35} \mathrm{H}_{36} \mathrm{O}, 472.2766$; found 472.2765 .

2,7-dibromo-9-(4-methoxyphenyl)-10-phenylphenanthrene (3id)<smiles>COc1ccc(-c2c(-c3ccccc3)c3cc(Br)ccc3c3ccc(Br)cc23)cc1</smiles> 
Purified by flash column chromatography (silica gel), [Hexane /DCM = 10:1 (v/v)] to give 3id as a white solid $(0.087 \mathrm{mmol}, 45.1 \mathrm{mg}$, $87 \%$ yield); ${ }^{1} \mathrm{H}-\mathrm{NMR}\left(600 \mathrm{MHz}, \mathrm{CDCl}_{3}\right) \delta 8.81(\mathrm{~d}, J=8.2 \mathrm{~Hz}, 2 \mathrm{H}), 7.66-7.71(\mathrm{~m}, 2 \mathrm{H}), 7.48-7.61(\mathrm{~m}, 3 \mathrm{H}), 7.39(\mathrm{~d}, J=2.3 \mathrm{~Hz}, 1 \mathrm{H}), 7.24-$ $7.34(\mathrm{~m}, 3 \mathrm{H}), 7.17(\mathrm{td}, J=4.6,2.4 \mathrm{~Hz}, 2 \mathrm{H}), 7.06(\mathrm{dd}, J=8.2,1.8 \mathrm{~Hz}, 1 \mathrm{H}), 6.77(\mathrm{~d}, J=8.2 \mathrm{~Hz}, 1 \mathrm{H}), 3.87(\mathrm{~s}, 3 \mathrm{H}) ;{ }^{13} \mathrm{C}-\mathrm{NMR}(100 \mathrm{MHz}$, $\left.\mathrm{CDCl}_{3}\right) \delta 154.3,139.3,137.8,135.6,135.3,133.2,131.7$ (8), 131.7 (5), 131.1, 131.0, 130.8, 130.0, 129.9 (8), 127.9, 127.6, 127.5, 126.7, 126.6 (5), 126.5, 126.4 (7), 122.5 (4), 122.4 (5), 111.0, 110.7, 56.1; HRMS (FD+): [m/z]: calcd. for $\mathrm{C}_{27} \mathrm{H}_{19} \mathrm{BrO}_{4} 438.0619$; found 438.0619 .

\section{2,7-dichloro-9-(4-methoxyphenyl)-10-phenylphenanthrene (3jd)}

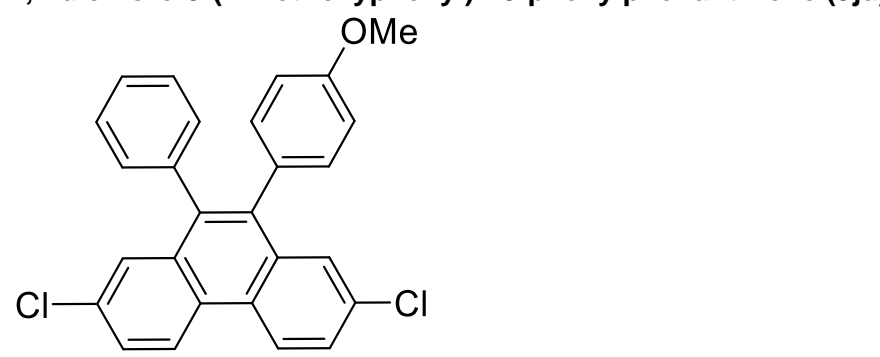

Purified by flash column chromatography (silica gel), [Hexane $/ \mathrm{DCM}=10: 1 \mathrm{(v/v)}$ ] to give 3jd as a white solid $(0.064 \mathrm{mmol}, 27.5 \mathrm{mg}$, $64 \%$ yield); ${ }^{1} \mathrm{H}-\mathrm{NMR}\left(600 \mathrm{MHz}, \mathrm{CDCl}_{3}\right) \delta 8.64(\mathrm{~d}, J=8.9 \mathrm{~Hz}, 2 \mathrm{H}), 7.61$ (qd, $\left.J=4.1,2.1 \mathrm{~Hz}, 2 \mathrm{H}\right), 7.55(\mathrm{~d}, J=2.1 \mathrm{~Hz}, 1 \mathrm{H}), 7.50(\mathrm{~d}, J=$ $2.7 \mathrm{~Hz}, 1 \mathrm{H}), 7.23-7.29(\mathrm{~m}, 6 \mathrm{H}), 7.11(\mathrm{dd}, \mathrm{J}=8.2,1.4 \mathrm{~Hz}, 2 \mathrm{H}), 7.01(\mathrm{dd}, \mathrm{J}=6.9,2.1 \mathrm{~Hz}, 2 \mathrm{H}), 6.78-6.80(\mathrm{~m}, 2 \mathrm{H}) ;{ }^{13} \mathrm{C}-\mathrm{NMR}(150 \mathrm{MHz}$, $\left.\mathrm{CDCl}_{3}\right) \delta 158.3,138.6,137.9,137.4,133.4,133.1,132.9$ (3), 132.9 (1), 131.8, 130.7, 130.6, 128.0, 127.9, 127.1 (9), 127.1 (6), 127.1 , 127.0, 126.9, 124.1, 113.3, 55.1; HRMS (FD+): [m/z]: calcd. for $\mathrm{C}_{27} \mathrm{H}_{18} \mathrm{Cl}_{2} \mathrm{O}$, 428.0735; found 428.0734.

\section{2-(10-phenylphenanthren-9-yl)thiophene (3ah) ${ }^{[4]}$}

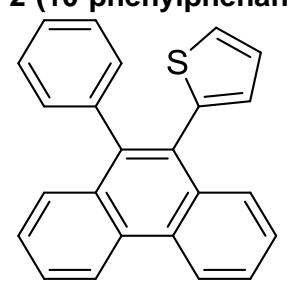

Purified by flash column chromatography (silica gel), [Hexane /DCM =10:1 (v/v)] to give 3af as a white solid $(0.093 \mathrm{mmol}, 31.3 \mathrm{mg}$, 93\% yield); ${ }^{1} \mathrm{H}$ NMR $\left(400 \mathrm{MHz}, \mathrm{CDCl}_{3}\right) \delta 8.80(\mathrm{~d}, J=8.2 \mathrm{~Hz}, 2 \mathrm{H}), 7.80(\mathrm{~d}, J=8.5,1 \mathrm{H}), 7.66-7.71(\mathrm{~m}, 2 \mathrm{H}), 7.53-7.57(\mathrm{~m}, 2 \mathrm{H}), 7.47-$ $7.51(\mathrm{~m}, 1 \mathrm{H}), 7.22-7.33(\mathrm{~m}, 6 \mathrm{H}), 6.96-6.93(\mathrm{~m}, 1 \mathrm{H}), 6.86(\mathrm{~m}, 1 \mathrm{H}) ;{ }^{13} \mathrm{C} \mathrm{NMR}\left(100 \mathrm{MHz}, \mathrm{CDCl}_{3}\right) \delta 140.0,139.8,139.4,132.4,131.6$, $130.7,130.3,129.9,129.6,129.1,128.1,127.6,127.5,126.8$ (8), 126.8 (6), 126.8, 126.7, 126.6, 126.3, 125.9, $122.5,122.4$.

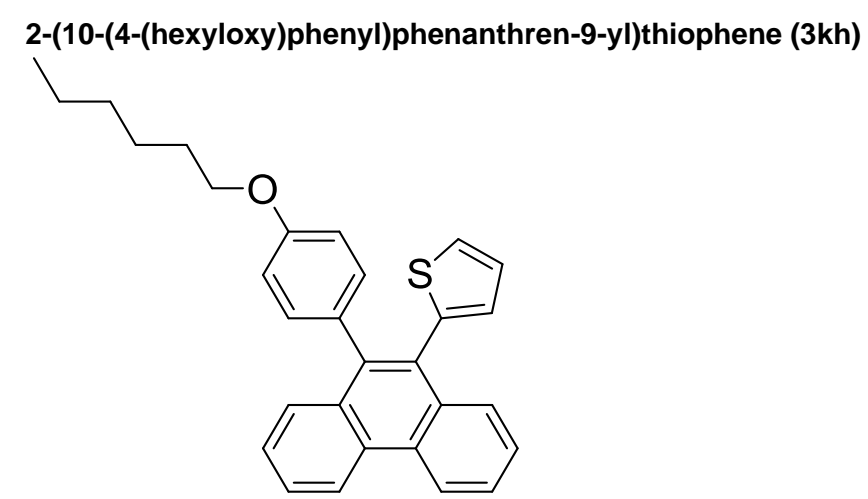

Purified by flash column chromatography (silica gel), [Hexane /DCM = 5:1 (v/v)] to give 3kh as a white solid $(0.05 \mathrm{mmol}, 21.8 \mathrm{mg}, 50 \%$ yield); ${ }^{1} \mathrm{H} \mathrm{NMR}\left(600 \mathrm{MHz}, \mathrm{CDCl}_{3}\right) \delta 8.79(\mathrm{~d}, J=8.2 \mathrm{~Hz}, 2 \mathrm{H}), 7.79(\mathrm{~d}, J=8.2 \mathrm{~Hz}, 1 \mathrm{H}), 7.67(\mathrm{dd}, J=8.2,6.9 \mathrm{~Hz}, 2 \mathrm{H}), 7.61(\mathrm{t}, J=7.2 \mathrm{~Hz}$, $1 \mathrm{H}), 7.52-7.55(\mathrm{~m}, 1 \mathrm{H}), 7.49(\mathrm{t}, J=7.6 \mathrm{~Hz}, 1 \mathrm{H}), 7.26-7.27(\mathrm{~m}, 1 \mathrm{H}), 7.12(\mathrm{~d}, J=8.2 \mathrm{~Hz}, 2 \mathrm{H}), 6.97-6.96(\mathrm{~m}, 1 \mathrm{H}), 6.83-6.87(\mathrm{~m}, 3 \mathrm{H}), 3.96$ $(\mathrm{t}, J=6.5 \mathrm{~Hz}, 2 \mathrm{H}), 1.77-1.82(\mathrm{~m}, 2 \mathrm{H}), 1.45-1.50(\mathrm{~m}, 2 \mathrm{H}), 1.35-1.37(\mathrm{~m}, 4 \mathrm{H}), 0.93(\mathrm{t}, J=6.9 \mathrm{~Hz}, 3 \mathrm{H}) ;{ }^{13} \mathrm{C} \mathrm{NMR}(150 \mathrm{MHz}, \mathrm{CDCl} 3) \delta$ 157.9, 140.3, 139.6, 132.5, 132.0, 131.7, 131.4, 130.4, 129.8 (0), 129.7 (9), 129.0, 128.2, 127.5, 126.8, 126.6, 126.5, 126.3, 125.9, 122.4 (4), 122.3 (5), 113.6, 67.9, 31.6, 29.3, 25.8, 22.6, 14.1, one $\mathrm{sp}^{2}$ carbon peak is not shown due to superimposition; HRMS (FD+): [m/z]: calcd. for $\mathrm{C}_{30} \mathrm{H}_{28} \mathrm{OS}, 436.1860$; found 436.1859 . 


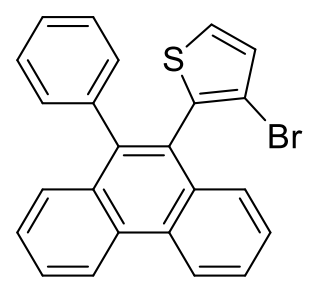

Purified by flash column chromatography (silica gel), [Hexane $/ \mathrm{DCM}=10: 1(\mathrm{v} / \mathrm{v})$ ] to give 3ai as a white solid $(0.082 \mathrm{mmol}, 34.1 \mathrm{mg}$, $82 \%$ yield); ${ }^{1} \mathrm{H}$ NMR $\left(400 \mathrm{MHz}, \mathrm{CDCl}_{3}\right) \delta 8.82(\mathrm{~d}, J=8.2 \mathrm{~Hz}, 2 \mathrm{H}), 7.69-7.74(\mathrm{~m}, 2 \mathrm{H}), 7.49-7.61(\mathrm{~m}, 4 \mathrm{H}), 7.30-7.39(\mathrm{~m}, 5 \mathrm{H}), 7.24(\mathrm{~d}, J$ $=5.5 \mathrm{~Hz}, 1 \mathrm{H}), 6.95(\mathrm{~d}, J=5.0 \mathrm{~Hz}, 1 \mathrm{H}) ;{ }^{13} \mathrm{C} \mathrm{NMR}\left(100 \mathrm{MHz}, \mathrm{CDCl}_{3}\right) \delta 140.9,138.8,136.2,131.6,131.1,130.8,130.6,129.9,129.8$, $129.4,128.1,128.0,127.5,127.4,127.2$ (4), 127.1 (9), 127.1, 127.0, 126.8, 126.7, $122.6(3), 122.6(0), 112.3$, one sp ${ }^{2}$ carbon peak is not shown due to superimposition. HRMS (FD+): [m/z]: calcd. for $\mathrm{C}_{24} \mathrm{H}_{15} \mathrm{BrS}, 414.0078$; found 414.0077 .

\section{2-bromo-5-(10-phenylphenanthren-9-yl)thiophene (3aj)}

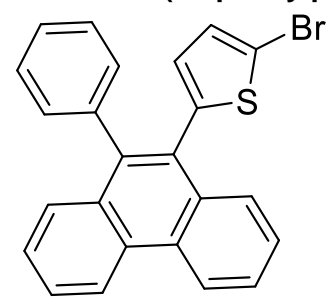

Purified by flash column chromatography (silica gel), [Hexane /DCM = 10:1 (v/v)] to give 3aj as a white solid $(0.085 \mathrm{mmol}, 35.3 \mathrm{mg}$, $85 \%$ yield); ${ }^{1} \mathrm{H}$ NMR (400 MHz, $\left.\mathrm{CDCl}_{3}\right) \delta 8.79$ (d, $\left.J=8.2 \mathrm{~Hz}, 2 \mathrm{H}\right), 7.83(\mathrm{~d}, J=8.2 \mathrm{~Hz}, 1 \mathrm{H}), 7.67-7.71(\mathrm{~m}, 2 \mathrm{H}), 7.49-7.59(\mathrm{~m}, 3 \mathrm{H}), 7.36-$ $7.33(\mathrm{~m}, 3 \mathrm{H}), 7.22(\mathrm{dd}, J=7.8,1.4 \mathrm{~Hz}, 2 \mathrm{H}), 6.88(\mathrm{~d}, J=3.7 \mathrm{~Hz}, 1 \mathrm{H}), 6.60(\mathrm{~d}, J=3.7 \mathrm{~Hz}, 1 \mathrm{H}) ;{ }^{13} \mathrm{C} \mathrm{NMR}\left(100 \mathrm{MHz}, \mathrm{CDCl}_{3}\right) \delta 141.9$, 140.2, 139.0, 132.0, 131.5, 130.5, 130.4, 129.9, 129.5, 129.3, 128.5, 128.2, 127.8, 127.3, 127.2, 127.0 (3), 127.0 (0), 126.8, 126.6, $122.5,112.1$, one $\mathrm{sp}^{2}$ carbon peak is not shown due to superimposition. HRMS (FD+): [m/z]: calcd. for $\mathrm{C}_{24} \mathrm{H}_{15} \mathrm{BrS}, 414.0078$; found 414.0078 .

\section{4-(10-(5-bromothiophen-2-yl)phenanthren-9-yl)phenyl hexanoate (3kj)} $\mathrm{HexO}$<smiles>Cc1ccc(-c2c(-c3ccc(Br)s3)c3ccccc3c3ccccc23)cc1</smiles>

Purified by flash column chromatography (silica gel), [Hexane /DCM = 5:1 (v/v)] to give $\mathbf{3 k j}$ as a white solid $(0.06 \mathrm{mmol}, 31.8 \mathrm{mg}, 60 \%$ yield); ${ }^{1} \mathrm{H}$ NMR $\left(600 \mathrm{MHz}, \mathrm{CDCl}_{3}\right) \delta 8.78(\mathrm{~d}, J=8.2 \mathrm{~Hz}, 2 \mathrm{H}), 7.82(\mathrm{~d}, J=8.2 \mathrm{~Hz}, 1 \mathrm{H}), 7.67-7.69(\mathrm{~m}, 2 \mathrm{H}), 7.55-7.59(\mathrm{~m}, 1 \mathrm{H}), 7.49(\mathrm{t}, J$ $=7.6 \mathrm{~Hz}, 1 \mathrm{H}), 7.11-7.13(\mathrm{~m}, 2 \mathrm{H}), 6.91(\mathrm{~d}, J=3.4 \mathrm{~Hz}, 1 \mathrm{H}), 6.88(\mathrm{~d}, J=8.2 \mathrm{~Hz}, 1 \mathrm{H}), 6.61(\mathrm{~d}, J=3.4 \mathrm{~Hz}, 1 \mathrm{H}), 4.00(\mathrm{t}, J=6.5 \mathrm{~Hz}, 2 \mathrm{H})$, 1.79-1.84 (m, 2H), 1.48-1.52 (m, 2H), 1.36-1.40 (m, 4H), $0.93(\mathrm{t}, J=6.9 \mathrm{~Hz}, 3 \mathrm{H}) ;{ }^{13} \mathrm{C}$ NMR $\left(150 \mathrm{MHz}, \mathrm{CDCl}_{3}\right) \delta 158.0,142.2,140.0$, 132.1, 131.9, 131.5, 130.9, 130.5, 129.8, 129.4, 129.3, 128.7, 128.3, 127.2, 127.1, 126.9, 126.7, 126.6, 122.4, 113.8, 112.0, 67.9, 31.6,

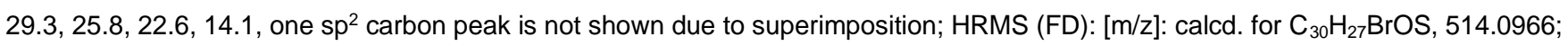
found 514.0964 .

\section{3,4-dibromo-2-(10-phenylphenanthren-9-yl)thiophene (3ak)}<smiles>Brc1csc(-c2c(-c3ccccc3)c3ccccc3c3ccccc23)c1Br</smiles>

Purified by flash column chromatography (silica gel), [Hexane $/ \mathrm{DCM}=10: 1(\mathrm{v} / \mathrm{v})$ ] to give 3af as a white solid $(0.071 \mathrm{mmol}, 35.1 \mathrm{mg}$, $71 \%$ yield); ${ }^{1} \mathrm{H}$ NMR $\left(400 \mathrm{MHz}, \mathrm{CDCl}_{3}\right) \delta 8.82(\mathrm{~d}, J=8.2 \mathrm{~Hz}, 2 \mathrm{H}), 7.72(\mathrm{td}, J=7.6,1.4 \mathrm{~Hz}, 2 \mathrm{H}), 7.51-7.61(\mathrm{~m}, 4 \mathrm{H}), 7.29-7.39(\mathrm{~m}, 6 \mathrm{H})$; ${ }^{13} \mathrm{C} \mathrm{NMR}\left(150 \mathrm{MHz}, \mathrm{CDCl}_{3}\right) \delta 141.0,138.4,137.4,131.4,130.9,130.6,130.5,130.0,129.6,128.2,127.5(0), 127.4(8), 127.5,127.4$, 
127.3, 126.9, 126.8, 126.7, 123.9, 122.7, 122.6, 115.8, 112.7, one $\mathrm{sp}^{2}$ carbon peak is not shown due to superimposition. HRMS (FD+): [m/z]: calcd. for $\mathrm{C}_{24} \mathrm{H}_{14} \mathrm{Br}_{2} \mathrm{~S}, 491.9183$; found 491.9182 .

2,3-dibromo-5-(10-phenylphenanthren-9-yl)thiophene (3al)

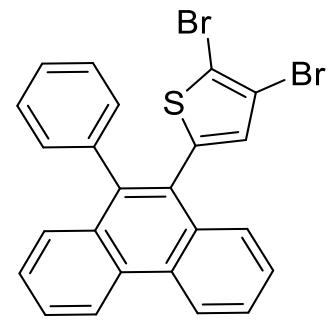

Purified by flash column chromatography (silica gel), [Hexane /DCM =10:1 (v/v)] to give 3al as a white solid $(0.075 \mathrm{mmol}, 37.1 \mathrm{mg}$, $75 \%$ yield); ${ }^{1} \mathrm{H}$ NMR $\left(400 \mathrm{MHz}, \mathrm{CDCl}_{3}\right) \delta 8.79(\mathrm{~d}, \mathrm{~J}=8.2 \mathrm{~Hz}, 2 \mathrm{H}), 7.83(\mathrm{~d}, \mathrm{~J}=8.2 \mathrm{~Hz}, 1 \mathrm{H}), 7.68-7.75(\mathrm{~m}, 2 \mathrm{H}), 7.48-7.62(\mathrm{~m}, 3 \mathrm{H}), 7.36-$ $7.41(\mathrm{~m}, 3 \mathrm{H}), 7.21-7.26(\mathrm{~m}, 2 \mathrm{H}), 6.70(\mathrm{~s}, 1 \mathrm{H}) ;{ }^{13} \mathrm{C} \mathrm{NMR}\left(100 \mathrm{MHz}, \mathrm{CDCl}_{3}\right) \delta$ 142.0, 140.5, 138.5, 131.6, 131.5, 131.3, 130.6, 130.5, 129.9, 128.3, 128.0, 127.5, 127.4, 127.3, 127.2, 127.0, 126.9, 126.8 (7), 122.6, 122.5, 112.9, 111.1. HRMS (FD+): [m/z]: calcd. for $\mathrm{C}_{24} \mathrm{H}_{14} \mathrm{Br}_{2} \mathrm{~S}, 491.9183$; found 491.9184 .

3-bromo-4-(2-bromophenyl)-2-(2,7-di-tert-butyl-10-phenylphenanthren-9-yl)thiophene (3hm)<smiles>CC(C)(C)c1ccc2c(c1)c(-c1ccccc1)c(-c1scc(-c3ccccc3Br)c1Br)c1cc(C(C)(C)C)ccc12</smiles>

Purified by flash column chromatography (silica gel), [Hexane /DCM = 10:1 (v/v)] to give $3 \mathrm{hm}$ as a white solid $(0.063 \mathrm{mmol}, 43.0 \mathrm{mg}$, $63 \%$ yield); ${ }^{1} \mathrm{H}$ NMR $\left(600 \mathrm{MHz}, \mathrm{CDCl}_{3}\right) \delta 8.71(\mathrm{~d}, J=8.2 \mathrm{~Hz}, 2 \mathrm{H}), 7.96(\mathrm{~d}, J=2.1 \mathrm{~Hz}, 1 \mathrm{H}), 7.76-7.79(\mathrm{~m}, 2 \mathrm{H}), 7.68(\mathrm{~d}, J=8.2 \mathrm{~Hz}, 1 \mathrm{H})$, $7.58(\mathrm{~d}, J=1.7 \mathrm{~Hz}, 1 \mathrm{H}), 7.40(\mathrm{t}, J=7.2 \mathrm{~Hz}, 2 \mathrm{H}), 7.34-7.37(\mathrm{~m}, 3 \mathrm{H}), 7.30-7.31(\mathrm{~m}, 2 \mathrm{H}), 7.25-7.28(\mathrm{~m}, 1 \mathrm{H}), 6.82(\mathrm{~s}, 1 \mathrm{H}), 1.42(\mathrm{~s}, 9 \mathrm{H})$, 1.31 (s, 9H); ${ }^{13} \mathrm{C}$ NMR $\left(150 \mathrm{MHz}, \mathrm{CDCl}_{3}\right) \delta 149.4,149.1,141.4,140.4,139.2,137.5,133.9,132.8,132.6,131.4,131.0,130.7,130.2$,

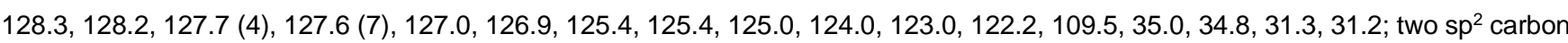
peaks are not shown due to superimposition. HRMS (FD+): [m/z]: calcd. for $\mathrm{C}_{38} \mathrm{H}_{34} \mathrm{Br}_{2} \mathrm{~S}, 680.0748$; found 680.0746.

\section{2-(10-phenylphenanthren-9-yl)benzofuran (3an)}<smiles>c1ccc(-c2c(-c3cc4ccccc4o3)c3ccccc3c3ccccc23)cc1</smiles>

Purified by flash column chromatography (silica gel), [Hexane /DCM = 10:1 (v/v)] to give 3an as a white solid $(0.06 \mathrm{mmol}, 22.2 \mathrm{mg}$, $60 \%$ yield); ${ }^{1} \mathrm{H}$ NMR $\left(400 \mathrm{MHz}, \mathrm{CDCl}_{3}\right) \delta 8.82-8.79(\mathrm{~m}, 1 \mathrm{H}), 7.62-7.74(\mathrm{~m}, 4 \mathrm{H}), 7.45-7.57(\mathrm{~m}, 4 \mathrm{H}), 7.17-7.31(\mathrm{~m}, 7 \mathrm{H}), 6.44(\mathrm{~s}, 1 \mathrm{H}) ;{ }^{13} \mathrm{C}$ NMR $\left(100 \mathrm{MHz}, \mathrm{CDCl}_{3}\right) \delta 154.7,153.9,140.8,139.0,131.4,131.3,130.8,130.2,129.9,128.6,128.4,127.8,127.4,127.1,127.1$, $126.8,126.7,126.5,123.7,122.5(8), 122.5$ (6), 122.5, 120.8, 111.1, 108.4, one sp ${ }^{2}$ carbon peak is not shown due to superimposition. HRMS (FD+): [m/z]: calcd. for $\mathrm{C}_{28} \mathrm{H}_{18} \mathrm{O}, 370.1358$; found 370.1357 .

\section{2-(10-phenylphenanthren-9-yl)benzo[b]thiophene (3ao)}




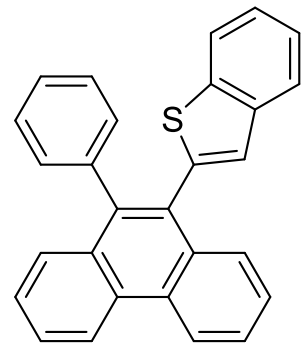

Purified by flash column chromatography (silica gel), [Hexane /DCM =10:1 (v/v)] to give 3ao as a white solid $(0.087 \mathrm{mmol}, 33.6 \mathrm{mg}$, 87\% yield); ${ }^{1} \mathrm{H}$ NMR (400 MHz, $\mathrm{CDCl}_{3}$ ) $\delta 8.85$ (dd, $\left.J=8.2,2.8 \mathrm{~Hz}, 2 \mathrm{H}\right), 7.83(\mathrm{~d}, J=8.2 \mathrm{~Hz}, 1 \mathrm{H}), 7.66-7.73(\mathrm{~m}, 2 \mathrm{H}), 7.61(\mathrm{~d}, J=8.2 \mathrm{~Hz}$, $1 \mathrm{H}), 7.52(\mathrm{t}, J=7.3 \mathrm{~Hz}, 1 \mathrm{H}), 7.44(\mathrm{q}, J=8.7 \mathrm{~Hz}, 2 \mathrm{H}), 7.14-7.33(\mathrm{~m}, 6 \mathrm{H}), 7.09(\mathrm{~s}, 1 \mathrm{H}), 7.01(\mathrm{t}, J=7.3 \mathrm{~Hz}, 1 \mathrm{H}), 6.92(\mathrm{~d}, J=7.3 \mathrm{~Hz}, 1 \mathrm{H})$; ${ }^{13} \mathrm{C}$ NMR $\left(100 \mathrm{MHz}, \mathrm{CDCl}_{3}\right) \delta 140.3,139.4,139.2,135.0,131.9,131.7,130.8,130.6,130.4,130.0,129.3,128.0,127.8,127.6,127.4$, $126.8,126.7,126.7,126.6,126.1,124.0,123.4,122.6,122.5,122.4$, one sp $^{2}$ carbon peak is not shown due to superimposition; HRMS (MALDI): [m/z]: calcd. for $\mathrm{C}_{28} \mathrm{H}_{18} \mathrm{~S}, 386.1129$; found 386.1131 .

\section{3-bromo-2-(10-phenylphenanthren-9-yl)benzo[b]thiophene (3ap)}<smiles>Brc1c(-c2c(-c3ccccc3)c3ccccc3c3ccccc23)sc2ccccc12</smiles>

Purified by flash column chromatography (silica gel), [Hexane /DCM =10:1 (v/v)] to give 3ap as a white solid $(0.08 \mathrm{mmol}, 37.2 \mathrm{mg}$, $80 \%$ yield); ${ }^{1} \mathrm{H}$ NMR $\left(400 \mathrm{MHz}, \mathrm{CDCl}_{3}\right) \delta 8.82(\mathrm{~d}, \mathrm{~J}=8.2 \mathrm{~Hz}, 2 \mathrm{H}), 7.69-7.79(\mathrm{~m}, 4 \mathrm{H}), 7.53-7.59(\mathrm{~m}, 4 \mathrm{H}), 7.42(\mathrm{~m}, 2 \mathrm{H}), 7.35(\mathrm{~m}, 3 \mathrm{H})$, $7.24(\mathrm{~m}, 2 \mathrm{H}) ;{ }^{13} \mathrm{C}$ NMR $\left(100 \mathrm{MHz}, \mathrm{CDCl}_{3}\right) \delta 140.7,139.2,138.4,137.6,136.9,131.6,130.9,130.6,130.3,129.9,129.7,128.1,128.0$, 127.5, 127.4, 127.3 (3), 127.2 (7), 126.9, 126.8, 125.1, 124.8, 123.2, 122.7, 122.6, 122.2, 110.0; HRMS (MALDI): [m/z]: calcd. for $\mathrm{C}_{28} \mathrm{H}_{17} \mathrm{BrS}, 464.0234$; found 464.0233 .

\section{Benzo[b]benzo[11,12]chryseno[6,5-d]thiophene (4ap)}

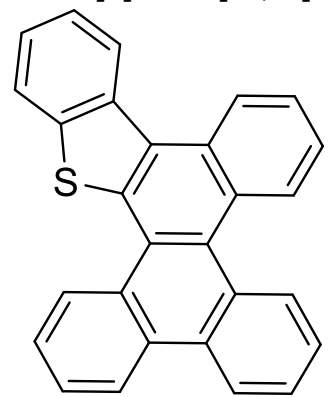

Purified by flash column chromatography (silica gel), [Hexane /DCM =10:1 (v/v)] to give 4ap as a white solid $(0.084 \mathrm{mmol}, 32.3 \mathrm{mg}$, $84 \%$ yield); ${ }^{1} \mathrm{H}$ NMR $\left(600 \mathrm{MHz}, \mathrm{CDCl}_{3}\right) \delta 9.27$ (d, $\left.J=6.9 \mathrm{~Hz}, 1 \mathrm{H}\right), 8.99(\mathrm{~d}, J=8.2 \mathrm{~Hz}, 1 \mathrm{H}), 8.82(\mathrm{t}, J=7.2 \mathrm{~Hz}, 2 \mathrm{H}), 8.68-8.75(\mathrm{~m}, 3 \mathrm{H})$, $8.04(\mathrm{~d}, J=7.6 \mathrm{~Hz}, 1 \mathrm{H}), 7.71-7.78(\mathrm{~m}, 3 \mathrm{H}), 7.59-7.67(\mathrm{~m}, 4 \mathrm{H}), 7.51(\mathrm{t}, J=7.6 \mathrm{~Hz}, 1 \mathrm{H}) ;{ }^{13} \mathrm{C} \mathrm{NMR}\left(150 \mathrm{MHz}, \mathrm{CDCl}_{3}\right) \delta 140.2,135.7$, 134.8, 130.7, 130.3, 129.9 (3), 129.9 (0), 129.8, 129.3, 129.2, 128.5, 127.4, 127.3 (5), 127.0, 126.9, 126.5, 125.8, 125.3, 125.0 (2), 124.9 (5), 124.3, 123.8, 123.5 (4), 123.5 (0), 122.5, three $\mathrm{sp}^{2}$ carbon peaks are not shown due to superimposition; HRMS (FD+): [m/z]: calcd. for $\mathrm{C}_{28} \mathrm{H}_{16} \mathrm{~S}, 384.0972$; found 384.0972 .

\section{2-methoxydibenzo[g,p]chrysene (4ad) ${ }^{[5]}$}<smiles>COc1ccc2c3ccccc3c3ccccc3c2c1</smiles> 
Purified by flash column chromatography (silica gel), [Hexane /DCM =10:1 (v/v)] to give 4ad as a white solid $(0.093 \mathrm{mmol}, 33.3 \mathrm{mg}$, 93\% yield); ${ }^{1} \mathrm{H}$ NMR (400 MHz, $\left.\mathrm{CDCl}_{3}\right) \delta 8.64-8.72(\mathrm{~m}, 7 \mathrm{H}), 8.11(\mathrm{~d}, J=2.8 \mathrm{~Hz}, 1 \mathrm{H}), 7.60-7.70(\mathrm{~m}, 6 \mathrm{H}), 7.27(\mathrm{dd}, J=8.8,2.8 \mathrm{~Hz}, 1 \mathrm{H})$, 4.07 (s, 3H); ${ }^{13} \mathrm{C} \operatorname{NMR}\left(100 \mathrm{MHz}, \mathrm{CDCl}_{3}\right) \delta$ 158.2, 132.4, 130.8, 130.5, 130.3 (9), 130.3 (6), 129.5, 129.3, 129.1, 128.8, 128.6, 127.5, 126.5 (4), $126.4(5), 126.4,126.2,126.1,125.7,123.6,123.5,115.3,105.8,55.4$, four sp ${ }^{2}$ carbon peaks are not shown due to superimposition.

\section{2,15-dimethoxydibenzo[g,p]chrysene $(4 \mathrm{fd})^{[5]}$

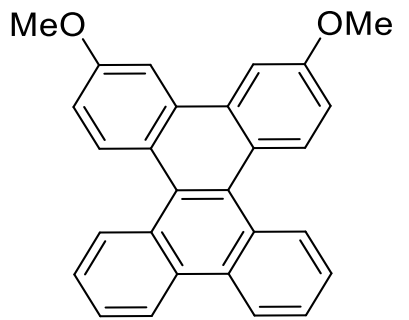

Purified by flash column chromatography (silica gel), [Hexane $/ D C M=10: 1(\mathrm{v} / \mathrm{v})$ ] to give $4 \mathbf{f d}$ as a white solid $(0.075 \mathrm{mmol}, 29.1 \mathrm{mg}$, $75 \%$ yield); ${ }^{1} \mathrm{H}$ NMR (400 MHz, $\left.\mathrm{CDCl}_{3}\right) \delta 8.60-8.69(\mathrm{~m}, 6 \mathrm{H}), 8.00(\mathrm{~d}, J=2.3 \mathrm{~Hz}, 2 \mathrm{H}), 7.58-7.65(\mathrm{~m}, 4 \mathrm{H}), 7.23-7.26(\mathrm{~m}, 2 \mathrm{H}), 4.05(\mathrm{~s}$, $6 \mathrm{H}) ;{ }^{13} \mathrm{C} \mathrm{NMR}\left(100 \mathrm{MHz}, \mathrm{CDCl}_{3}\right) \delta 158.1,131.9,130.6,130.4,129.2,128.6,126.4,126.0,125.9,123.9,123.5,115.2,106.1,55.5$; HRMS (FD+): [m/z]: calcd. for $\mathrm{C}_{34} \mathrm{H}_{22}, 430.1722$; found 502.1721 .

\section{2,5-bis(10-phenylphenanthren-9-yl)thiophene (5ah)}<smiles>c1ccc(-c2c(-c3ccc(-c4ccccc4-c4c(-c5ccccc5)c5ccccc5c5ccccc45)s3)c3ccccc3c3ccccc23)cc1</smiles>

Purified by flash column chromatography (silica gel), [Hexane /DCM = 10:1 (v/v)] to give 5ah as a white solid $(0.033 \mathrm{mmol}, 19.4 \mathrm{mg}$, $33 \%$ yield); ${ }^{1} \mathrm{H}$ NMR (600 MHz, $\left.\mathrm{CDCl}_{3}\right) \delta 8.78(\mathrm{~d}, J=8.2 \mathrm{~Hz}, 2 \mathrm{H}), 8.77(\mathrm{~d}, J=8.2 \mathrm{~Hz}, 2 \mathrm{H}), 7.66-7.70(\mathrm{~m}, 5 \mathrm{H}), 7.62(\mathrm{~d}, J=8.2 \mathrm{~Hz}, 2 \mathrm{H})$, 7.55 (t, $J=7.2 \mathrm{~Hz}, 2 \mathrm{H}), 7.49(\mathrm{t}, J=7.6 \mathrm{~Hz}, 2 \mathrm{H}), 7.39-7.44(\mathrm{~m}, 7 \mathrm{H}), 7.27(\mathrm{~d}, J=8.2 \mathrm{~Hz}, 4 \mathrm{H}), 6.61(\mathrm{~s}, 2 \mathrm{H}) ;{ }^{13} \mathrm{C} \mathrm{NMR}(100 \mathrm{MHz}, \mathrm{CDCl} 3)$ ठ 140.8, 139.7, 139.6, 132.4, 131.5, 130.9, 130.4, 129.8, 129.6, 128.4, 128.1, 127.6, 126.8, 126.7, 126.6, 126.5, 122.5, 122.3, two sp² carbon peaks are not shown due to superimposition; HRMS (FD+): [m/z]: calcd. for $\mathrm{C}_{44} \mathrm{H}_{28} \mathrm{~S}, 588.1912$; found 588.1912.

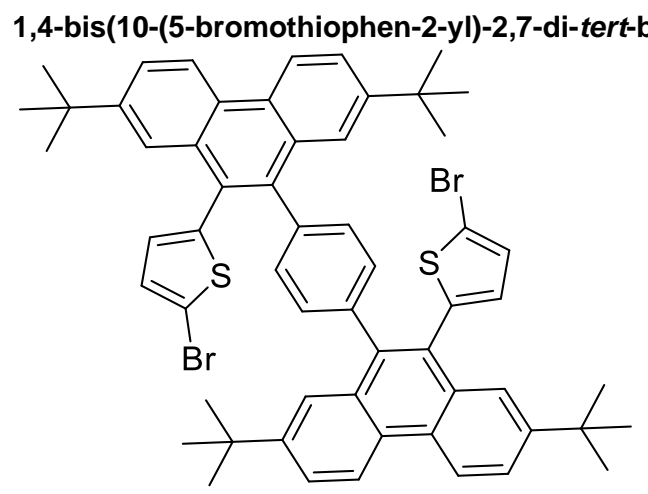

Purified by flash column chromatography (silica gel), [Hexane /DCM = 10:1 (v/v)] to give $5 \mathbf{l j}$ as a white solid $(0.075 \mathrm{mmol}, 41.0 \mathrm{mg}$, $42 \%$ yield); ${ }^{1} \mathrm{H}$ NMR $\left(600 \mathrm{MHz}, \mathrm{CDCl}_{3}\right) \delta 8.67-8.70(\mathrm{~m}, 4 \mathrm{H}), 7.76(\mathrm{~m}, 5 \mathrm{H}), 7.70(\mathrm{~d}, J=2.1 \mathrm{~Hz}, 1 \mathrm{H}), 7.55(\mathrm{~d}, J=2.1 \mathrm{~Hz}, 1 \mathrm{H}), 7.29(\mathrm{~s}$, 2H), $7.25(\mathrm{~d}, J=3.4 \mathrm{~Hz}, 3 \mathrm{H}), 7.06(\mathrm{~d}, J=3.4 \mathrm{~Hz}, 1 \mathrm{H}), 6.94(\mathrm{~d}, J=4.1 \mathrm{~Hz}, 1 \mathrm{H}), 6.66(\mathrm{~d}, J=3.4 \mathrm{~Hz}, 1 \mathrm{H}), 6.48(\mathrm{~d}, J=3.4 \mathrm{~Hz}, 1 \mathrm{H}), 1.32-$ 1.37 (m, 36H); ${ }^{13} \mathrm{C} \mathrm{NMR}\left(150 \mathrm{MHz}, \mathrm{CDCl}_{3}\right) \delta$ 149.3, 149.3, 149.2, 149.1, 142.8, 142.4, 139.9, 139.8, 137.9, 137.8, 131.9, 131.8, 131.0, 130.9, 130.2, 130.2, 129.7, 129.5 (2), 129.4 (9), 129.3, 128.9, 128.6, 128.5, 128.4, 127.7, 125.3, 125.1, 124.8 (8), 124.8 (5), 124.1 ,

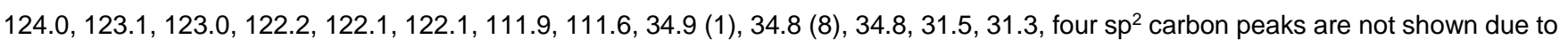
superimposition. HRMS (FD+): [m/z]: calcd. for $\mathrm{C}_{58} \mathrm{H}_{56} \mathrm{Br}_{2} \mathrm{~S}_{2}, 974.2190$; found 974.2189 . 
(9-(benzofuran-2-yl)-9H-fluoren-9-yl)(phenyl)methyl 2,2,2-trifluoroacetate (3an')<smiles>O=C(OC(c1ccccc1)C1(c2cc3ccccc3o2)c2ccccc2-c2ccccc21)C(F)(F)F</smiles>

Purified by flash column chromatography (silica gel), [Hexane /DCM = 10:1 (v/v)] to give 3an' as a white solid $(0.068 \mathrm{mmol}, 32.9 \mathrm{mg}$, $68 \%$ yield); ${ }^{1} \mathrm{H}$ NMR $\left(600 \mathrm{MHz}, \mathrm{CDCl}_{3}\right) \delta$ 8.05-8.09 (m, 1H), 7.77-7.81 (m, 1H), 7.58-7.62 (m, 2H), 7.42-7.54 (m, 4H), 7.29-7.34 (m, $3 \mathrm{H}), 7.18-7.21(\mathrm{~m}, 2 \mathrm{H}), 7.05(\mathrm{t}, J=7.6 \mathrm{~Hz}, 1 \mathrm{H}), 6.92(\mathrm{t}, J=7.6 \mathrm{~Hz}, 2 \mathrm{H}), 6.70(\mathrm{~d}, J=7.3 \mathrm{~Hz}, 2 \mathrm{H}), 6.39(\mathrm{~s}, 1 \mathrm{H}) ;{ }^{13} \mathrm{C} \mathrm{NMR}(150 \mathrm{MHz}$, $\left.\mathrm{CDCl}_{3}\right) \delta 156.0\left(\mathrm{q}, \mathrm{J}_{\mathrm{C}-\mathrm{F}}=42.2 \mathrm{~Hz}\right), 156.0,155.3,143.0,142.1,141.7,140.5,133.1,129.0,128.7,128.4,127.9,127.5,127.3,127.2$, $127.1,125.8,124.2,122.9,120.9,120.2,120.1,114.5$ (q, $\left.J_{C-F}=286.6 \mathrm{~Hz}\right), 111.1,104.9,82.0,59.8$, one sp ${ }^{2}$ carbon peak is not shown due to superimposition; HRMS (FD+): [m/z]: calcd. for $\mathrm{C}_{30} \mathrm{H}_{19} \mathrm{~F}_{3} \mathrm{O}_{3}, 484.1286$; found 484.1285. 
7. ${ }^{1} \mathrm{H}$ and ${ }^{13} \mathrm{C}$ NMR spectra of starting substrates and products

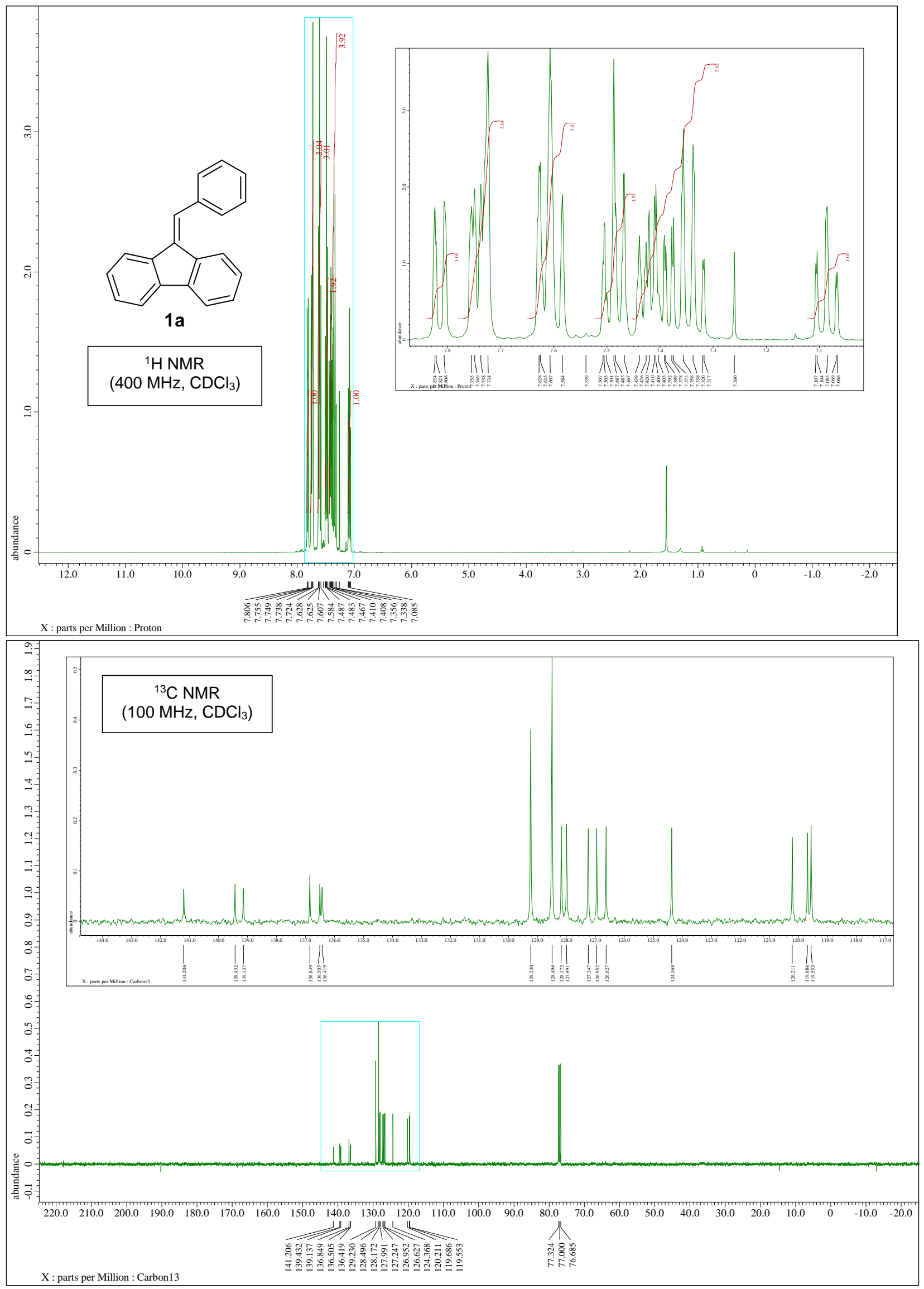



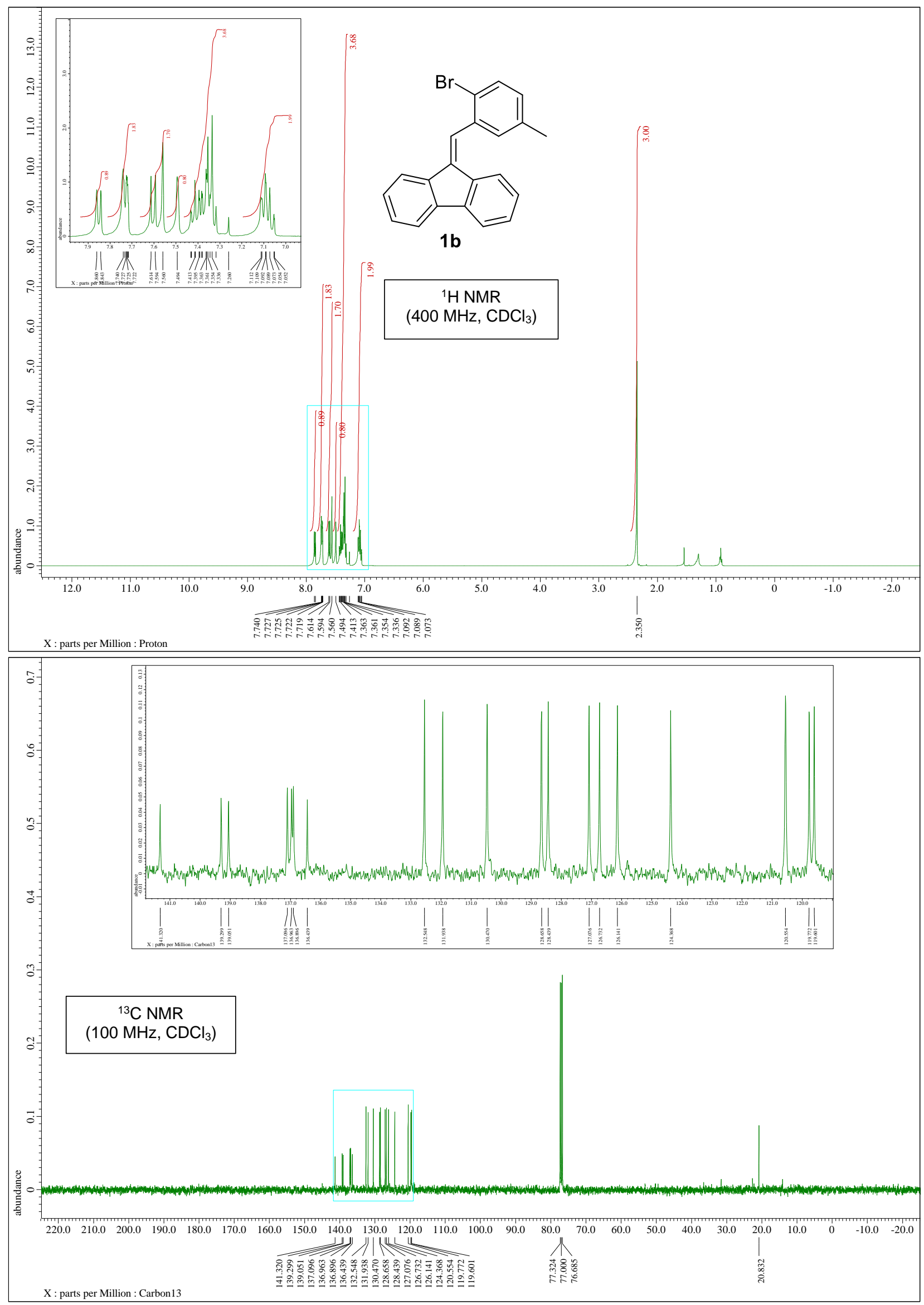


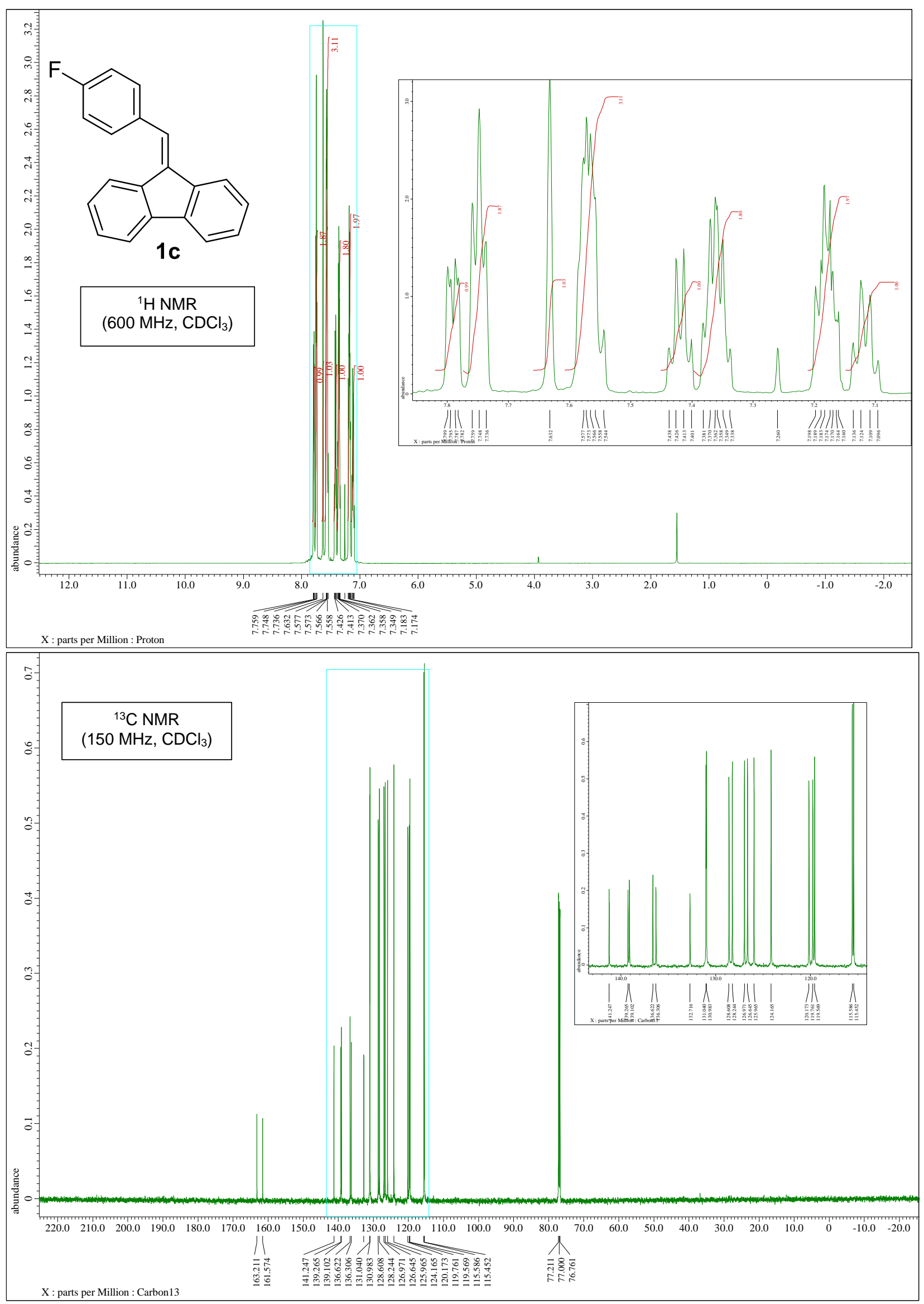



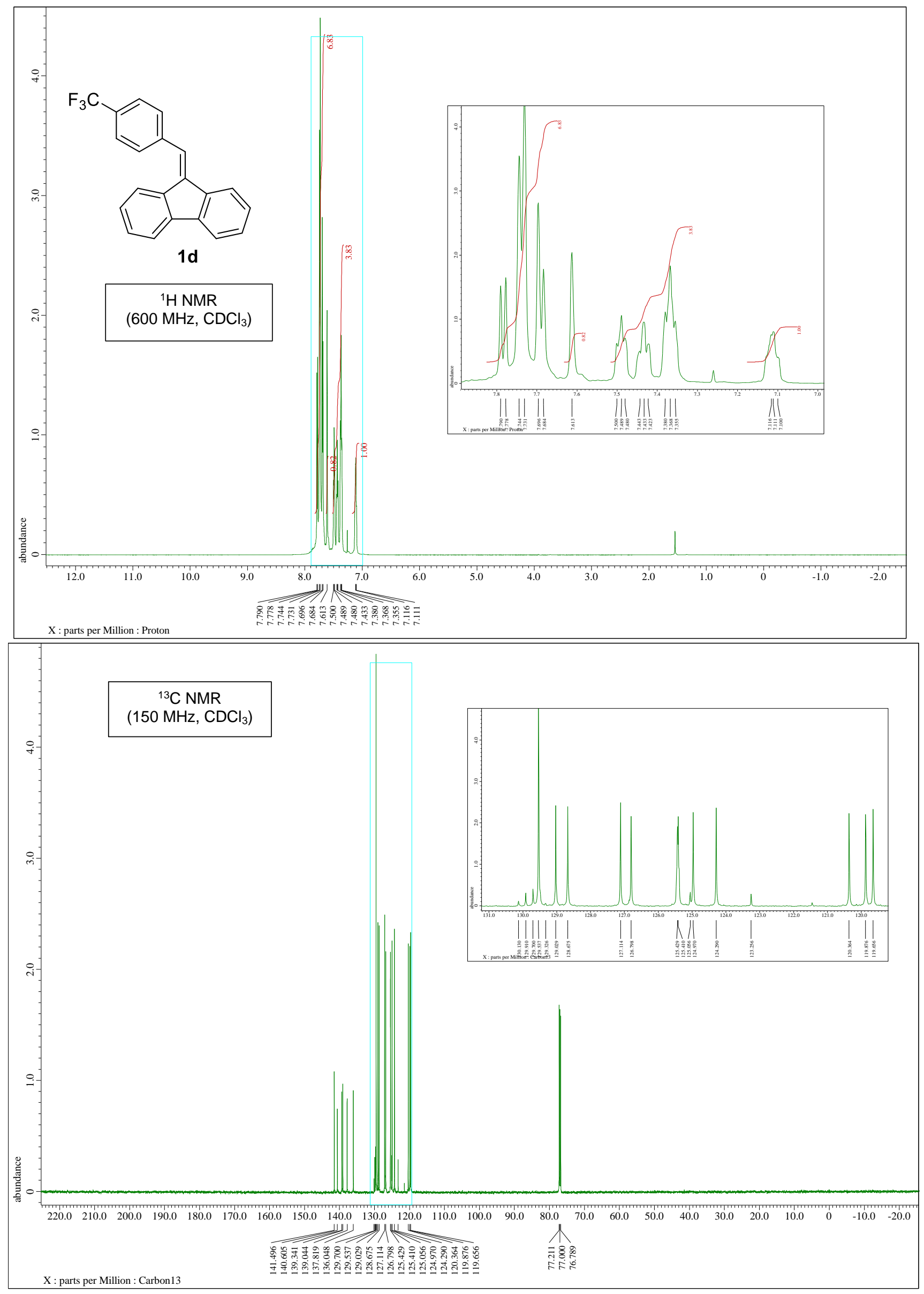

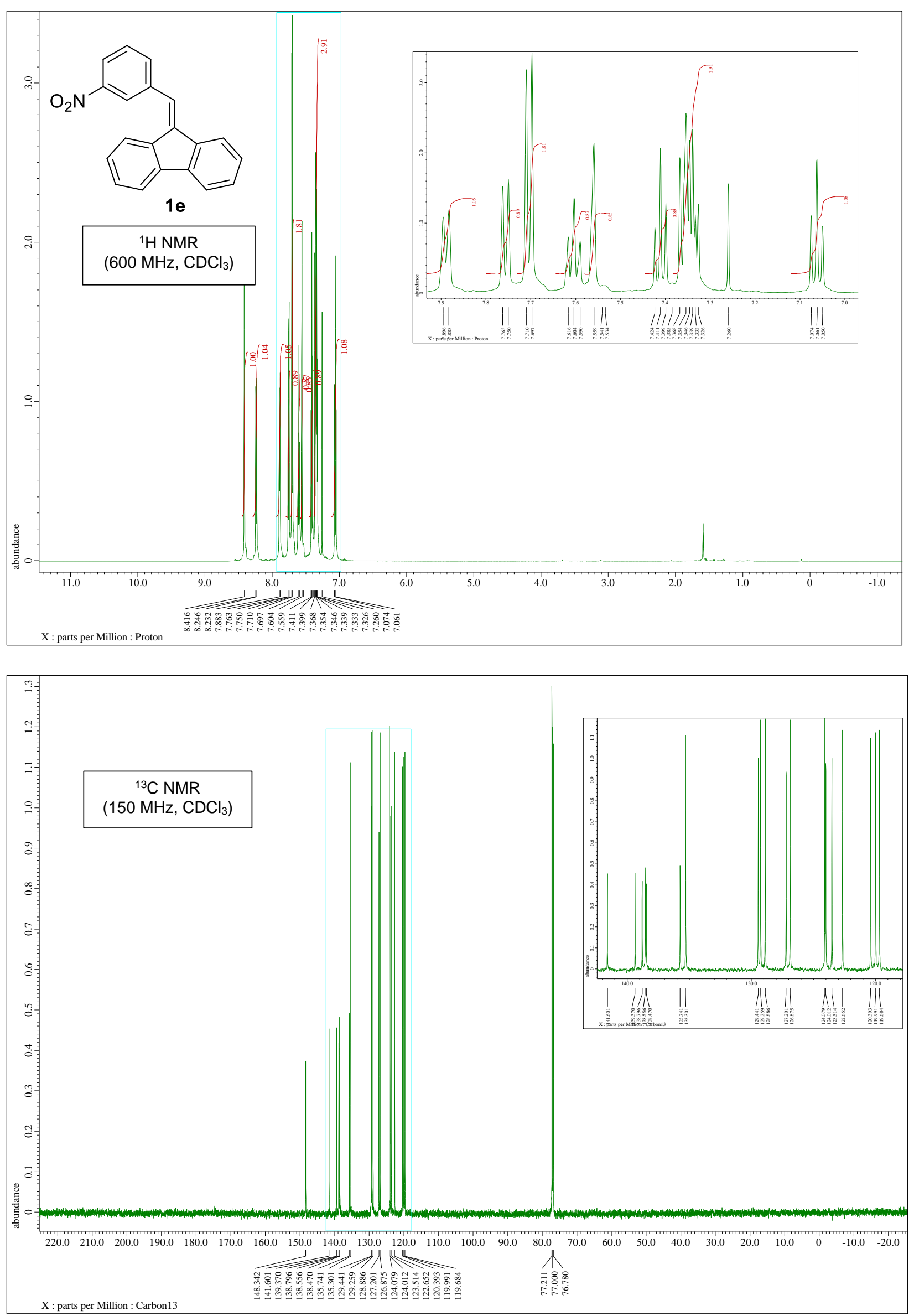

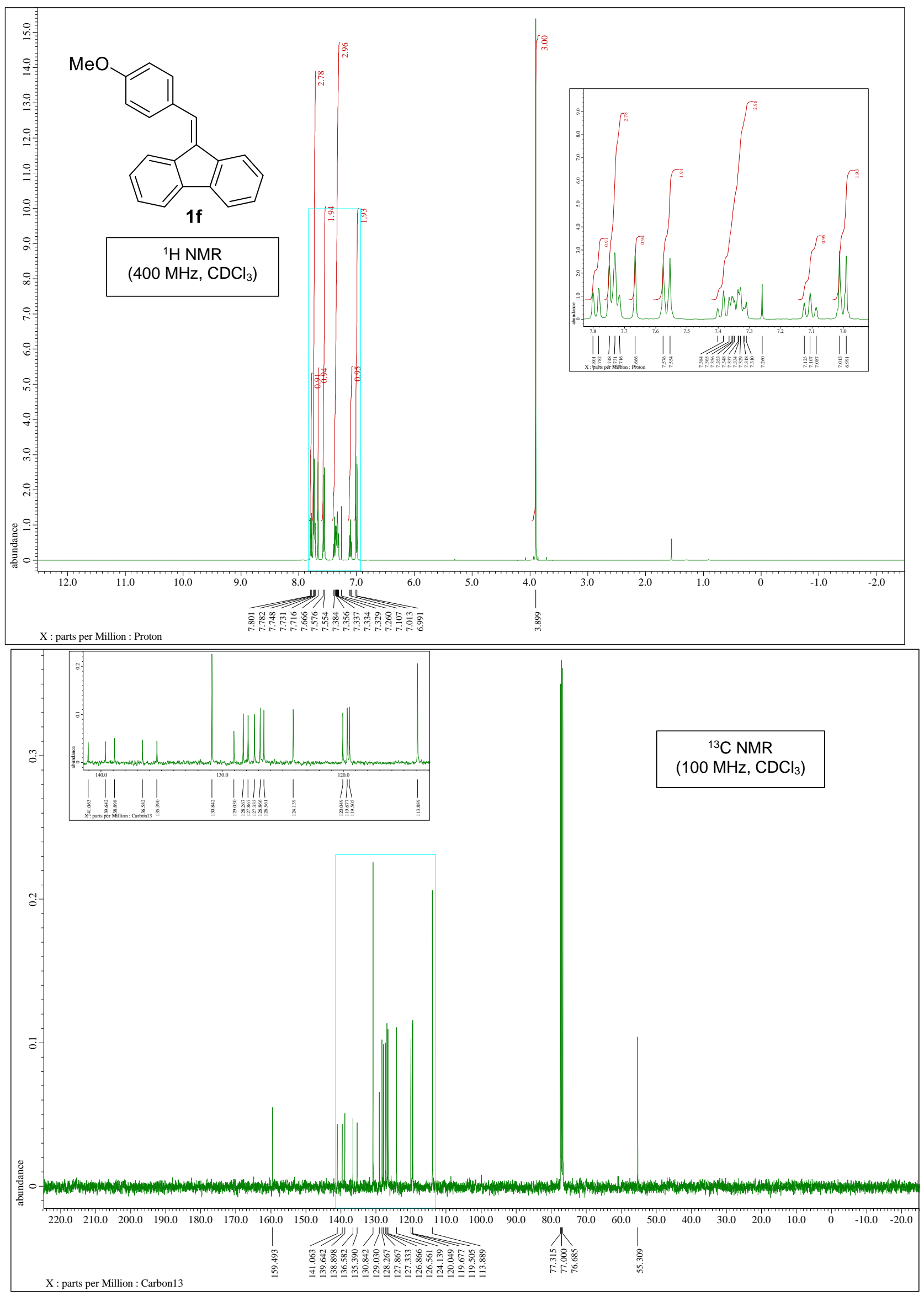

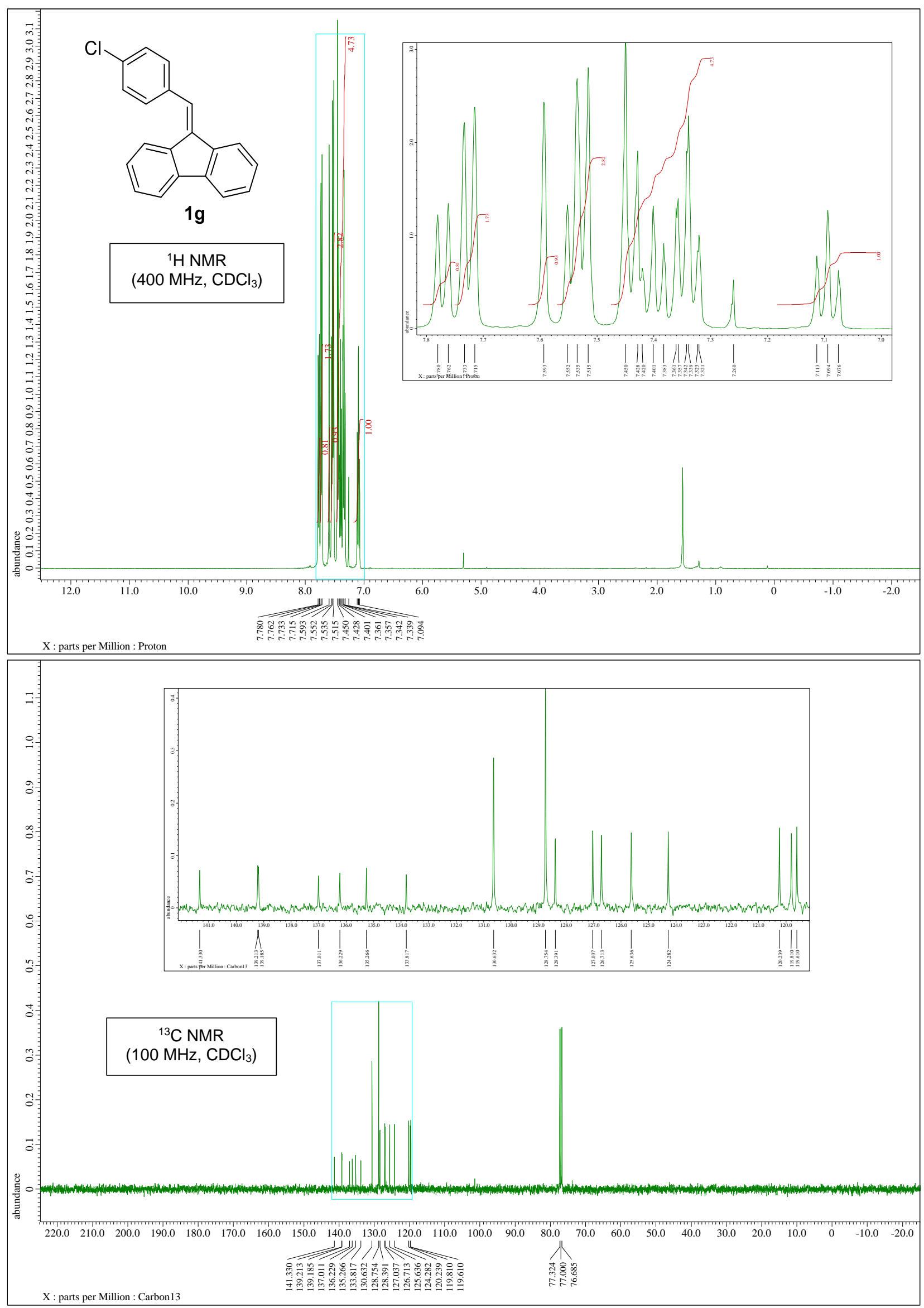

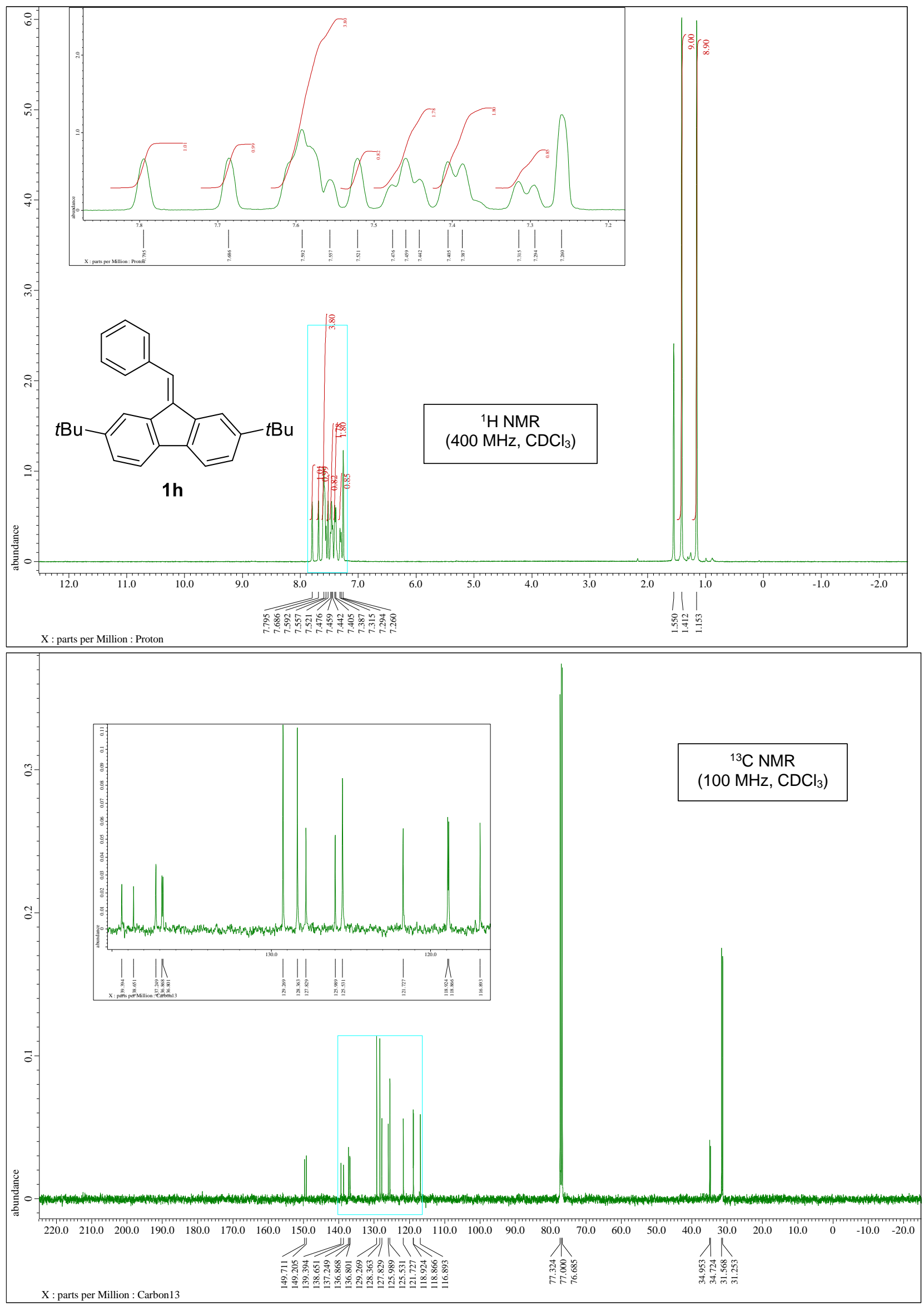

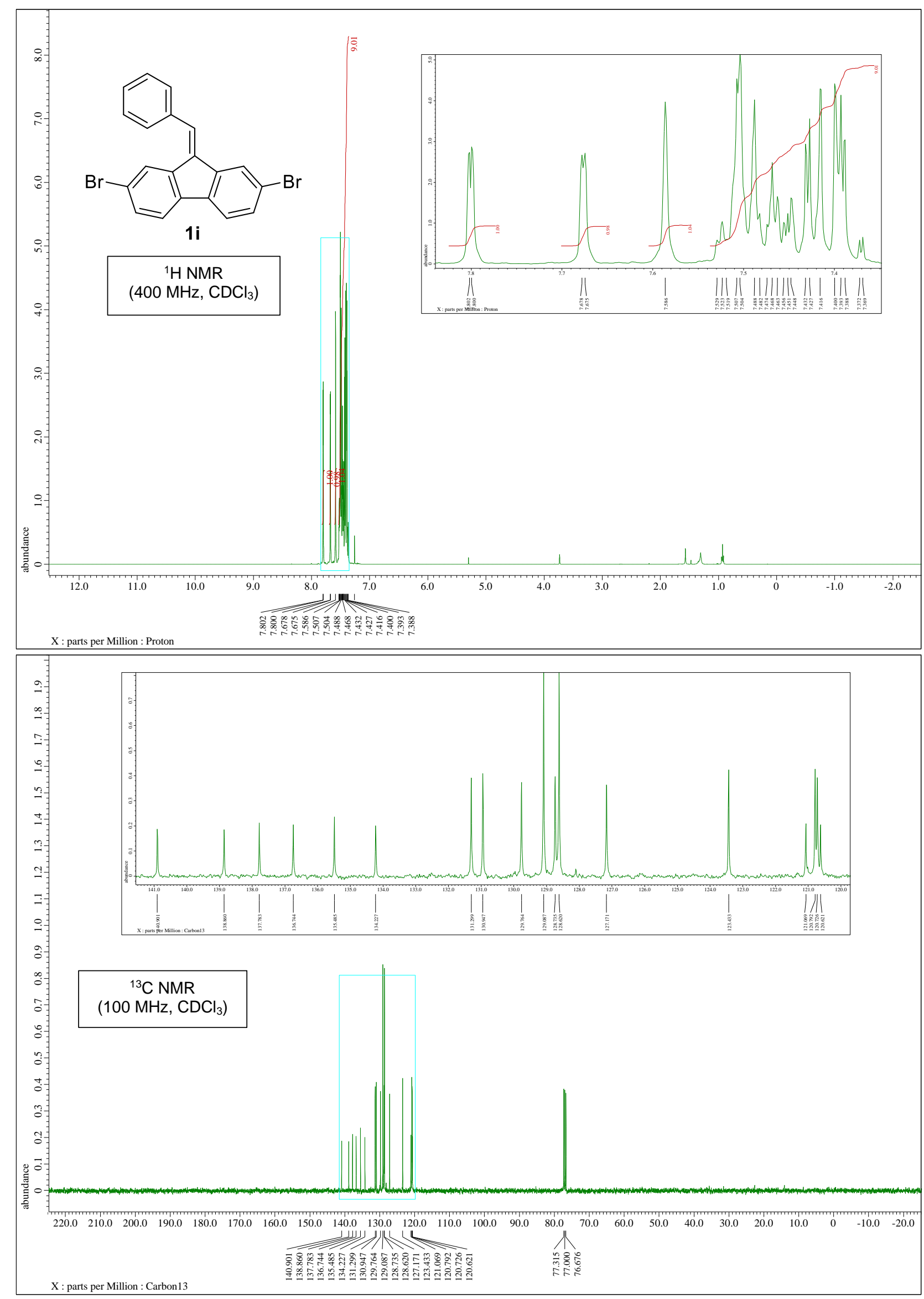

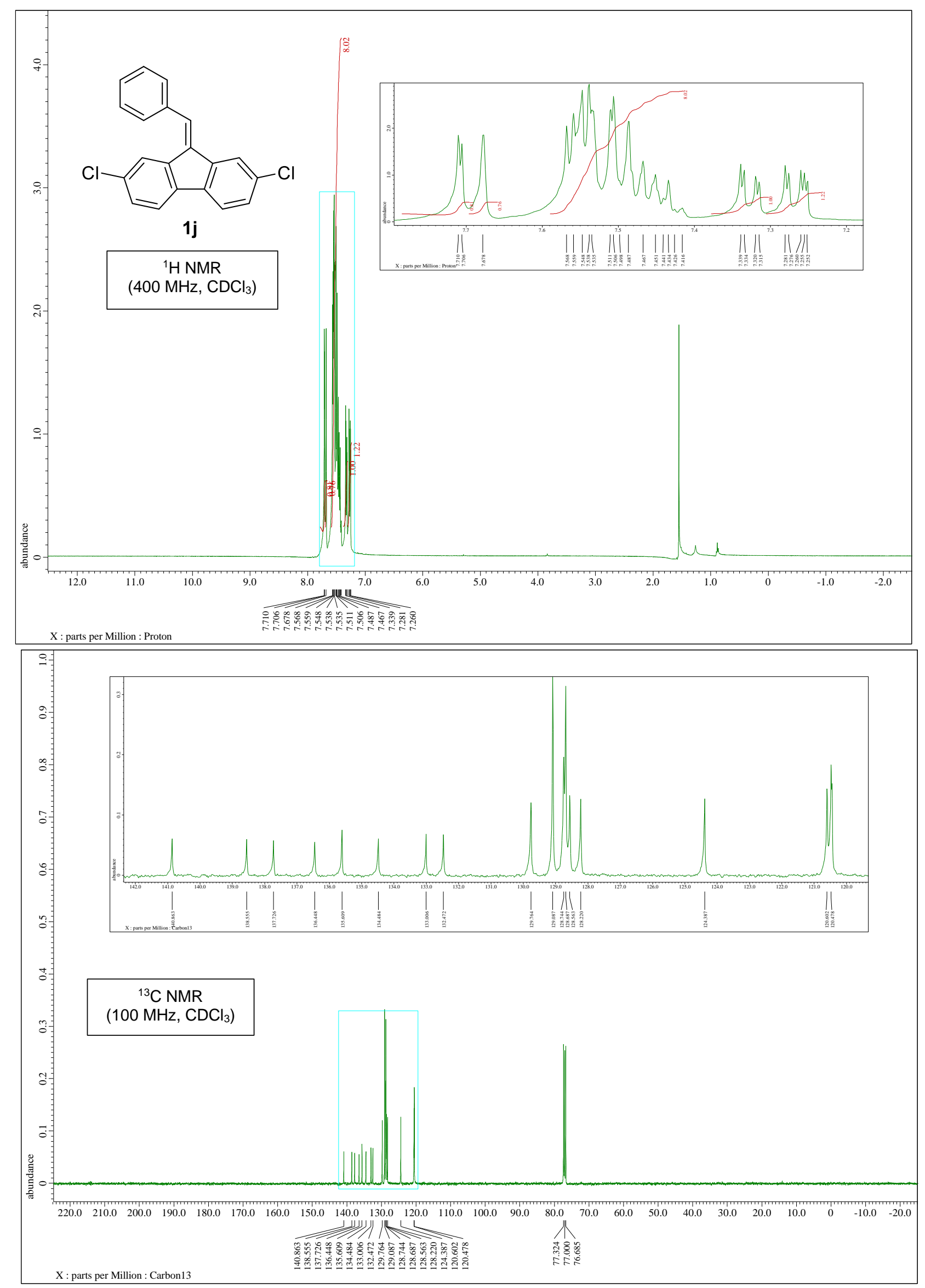

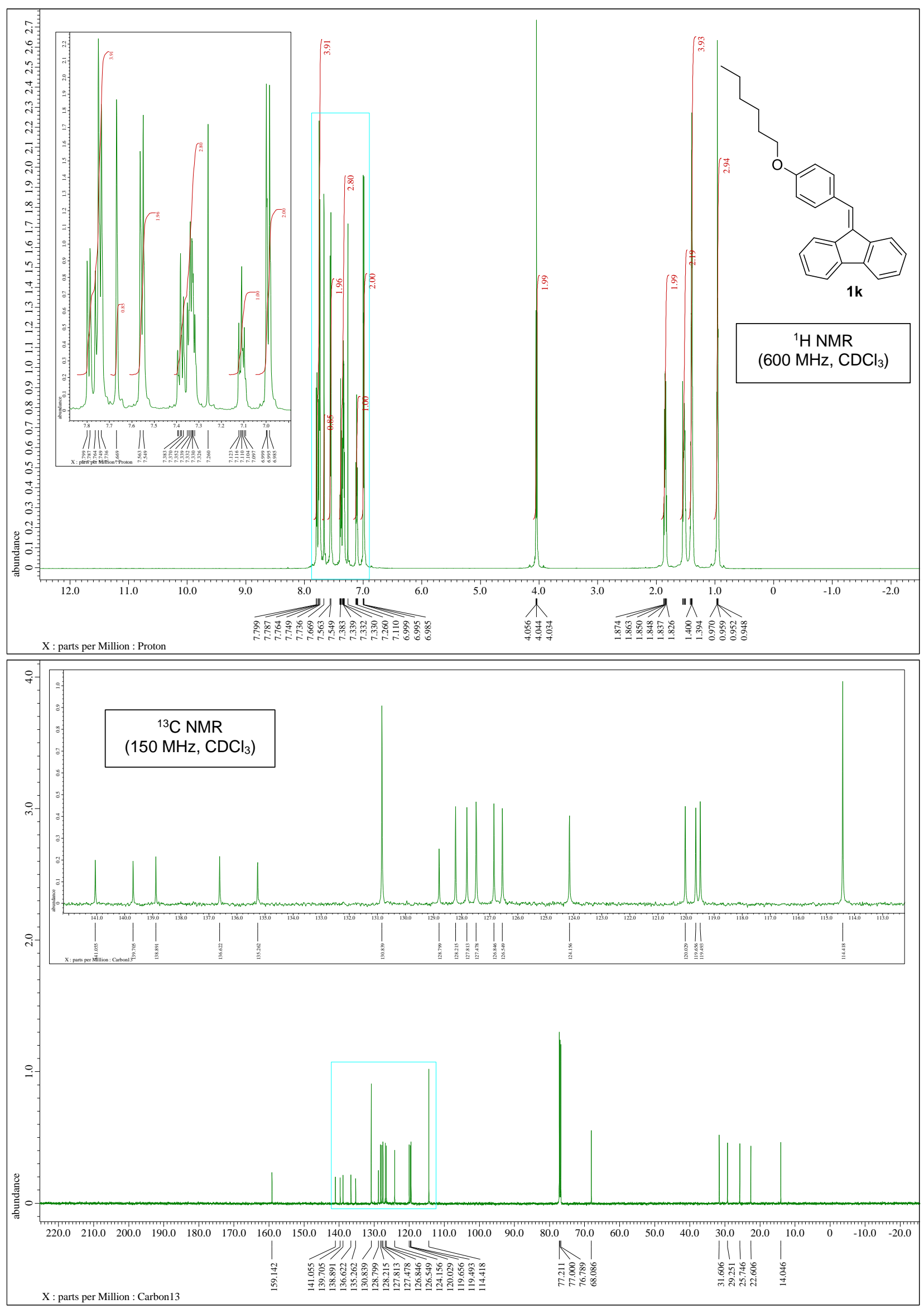

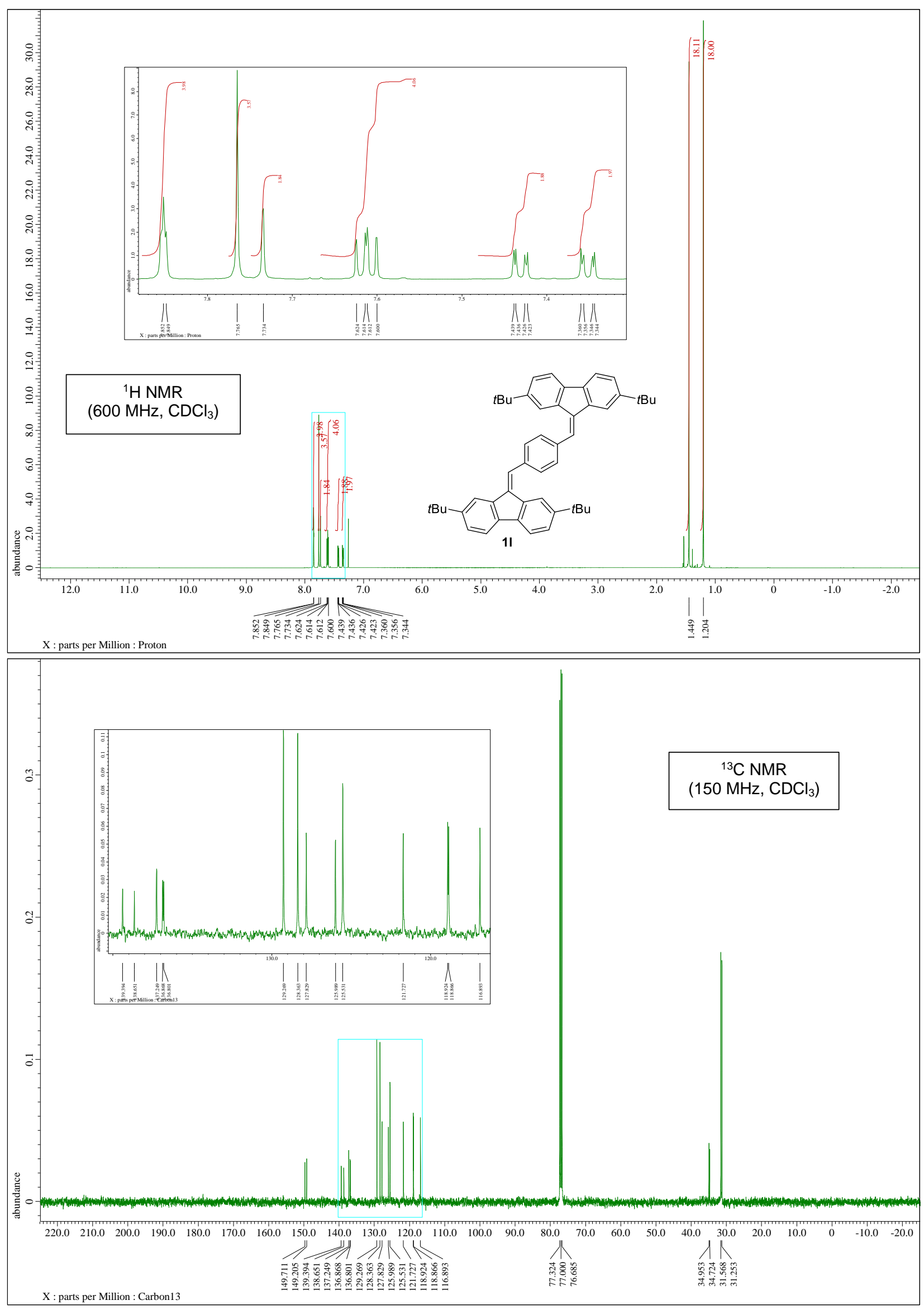

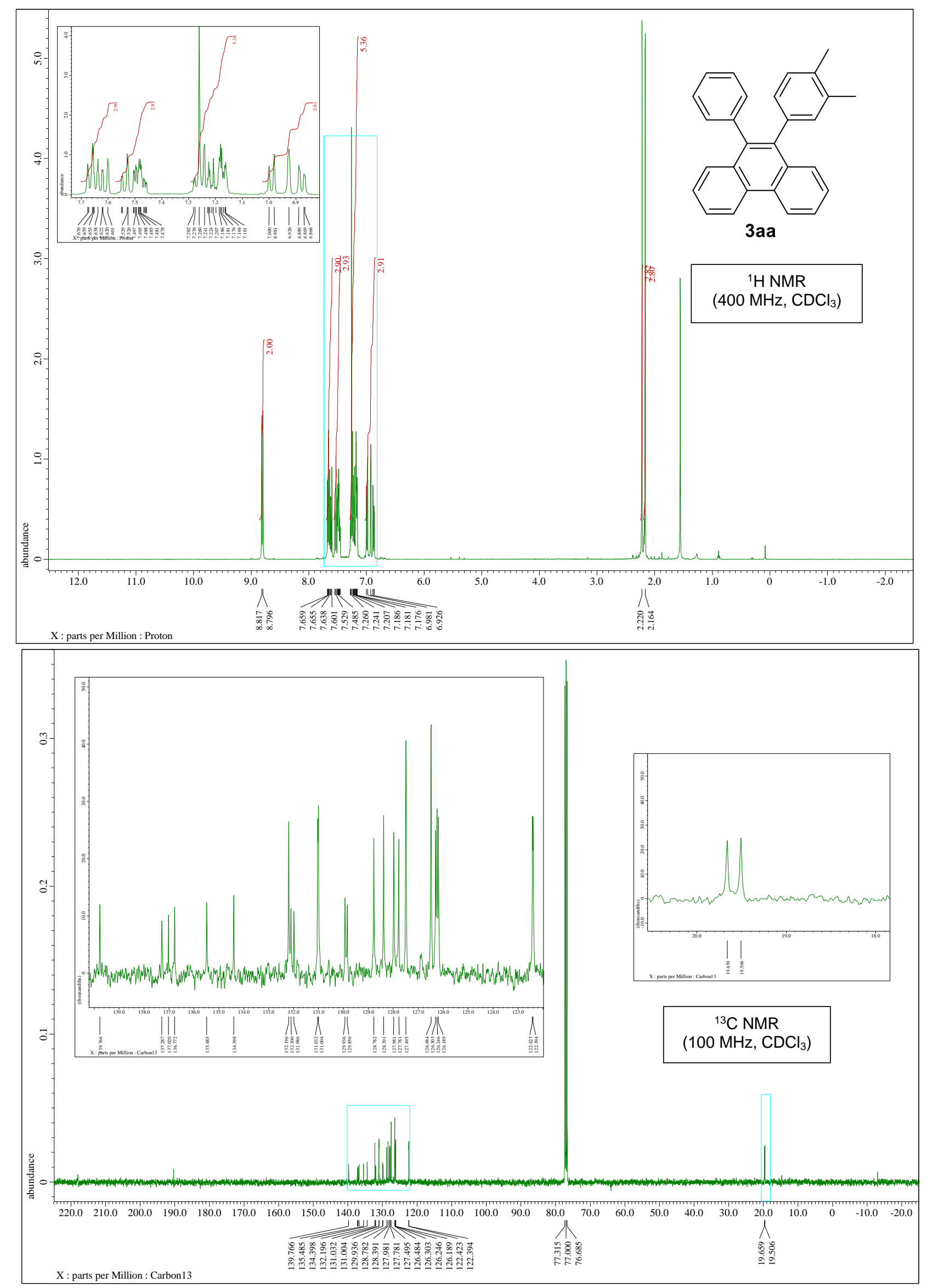

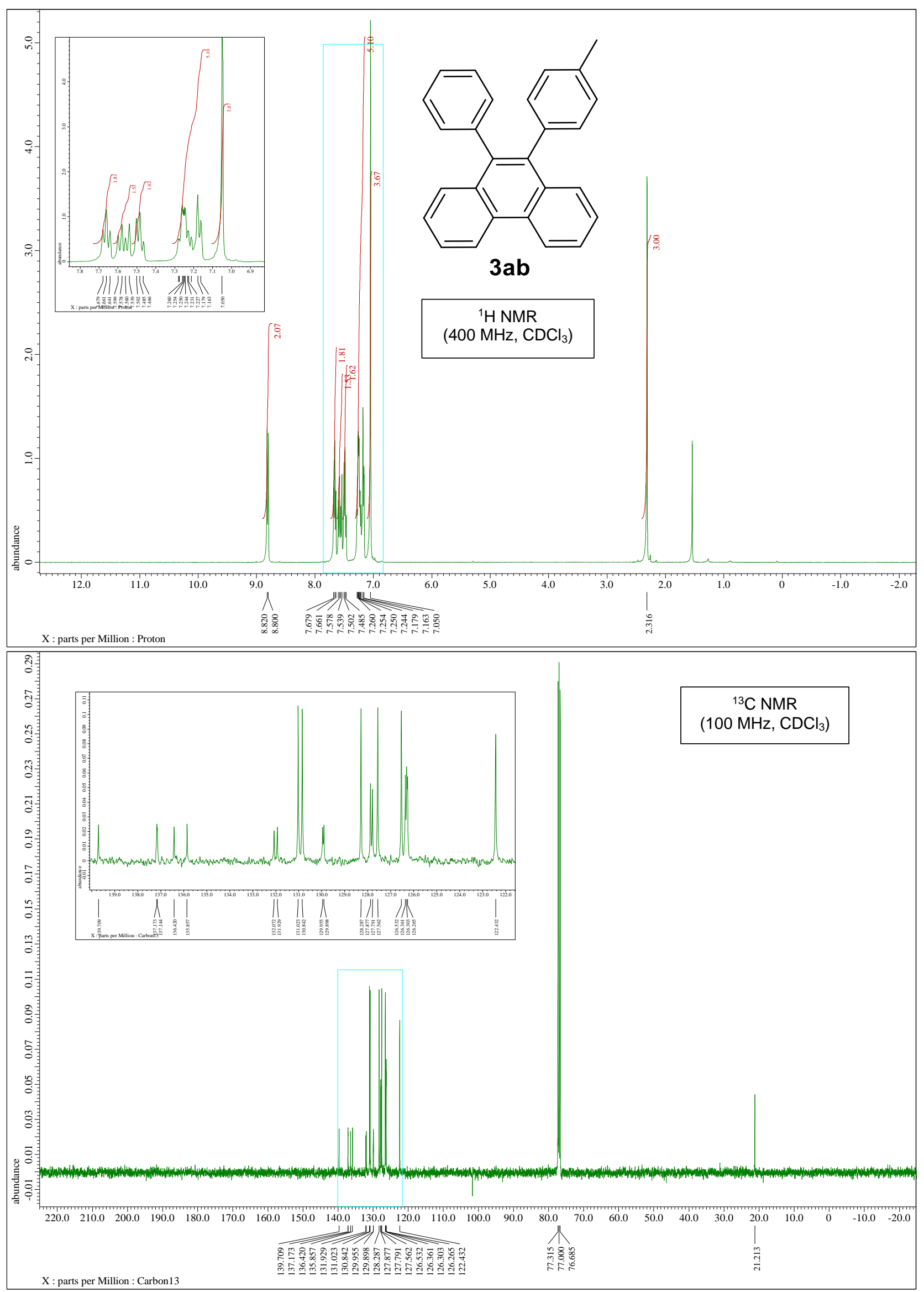

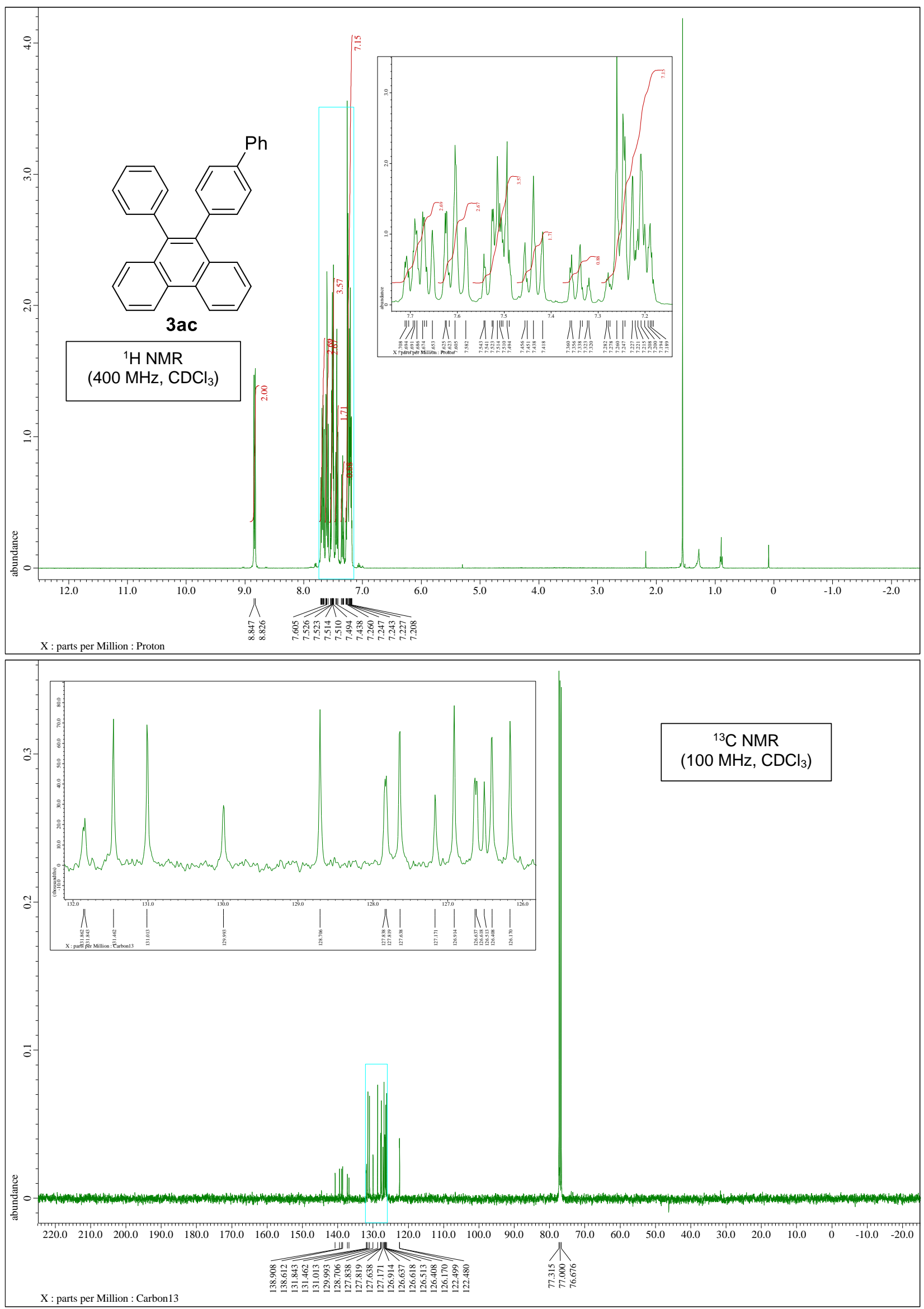

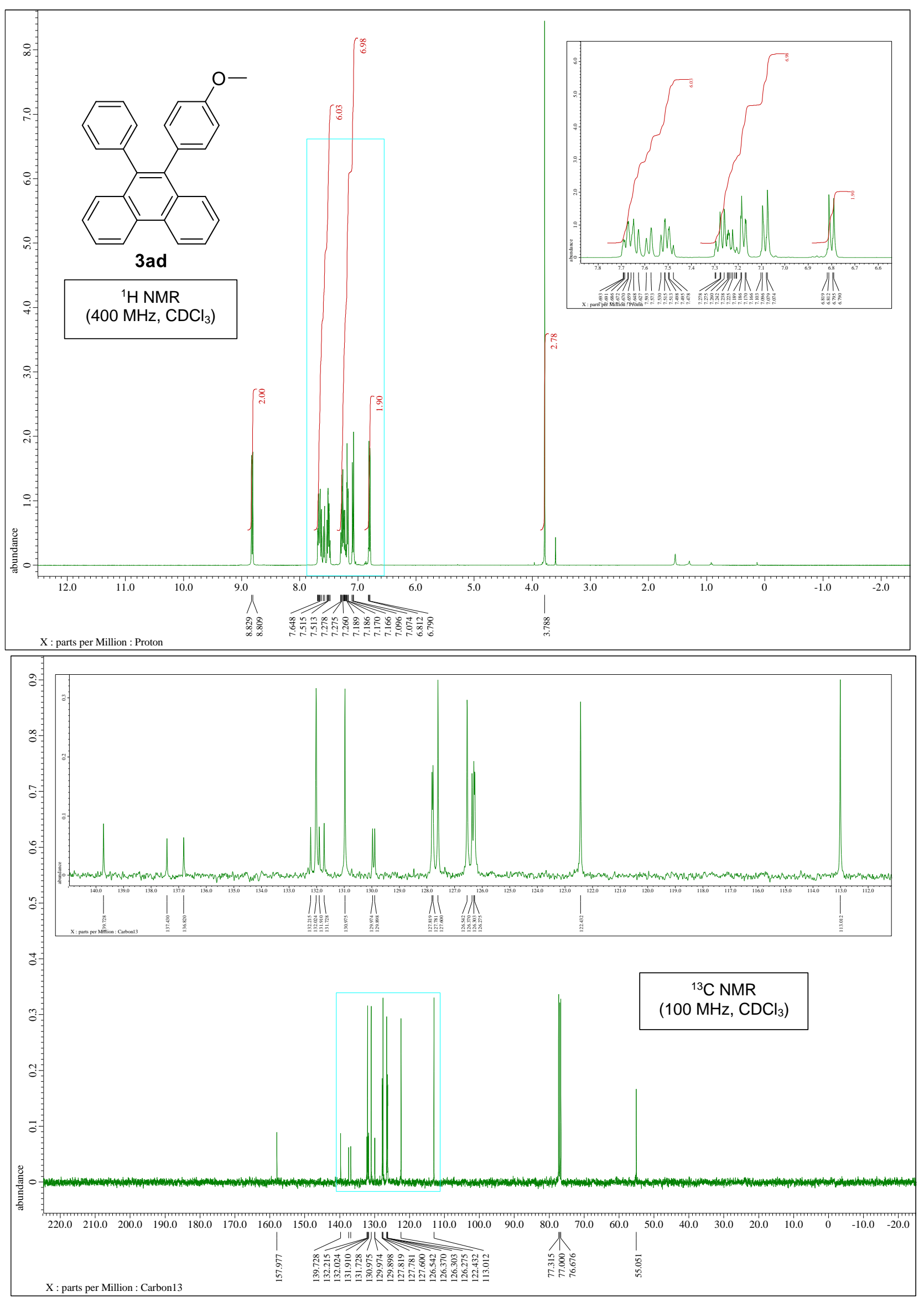

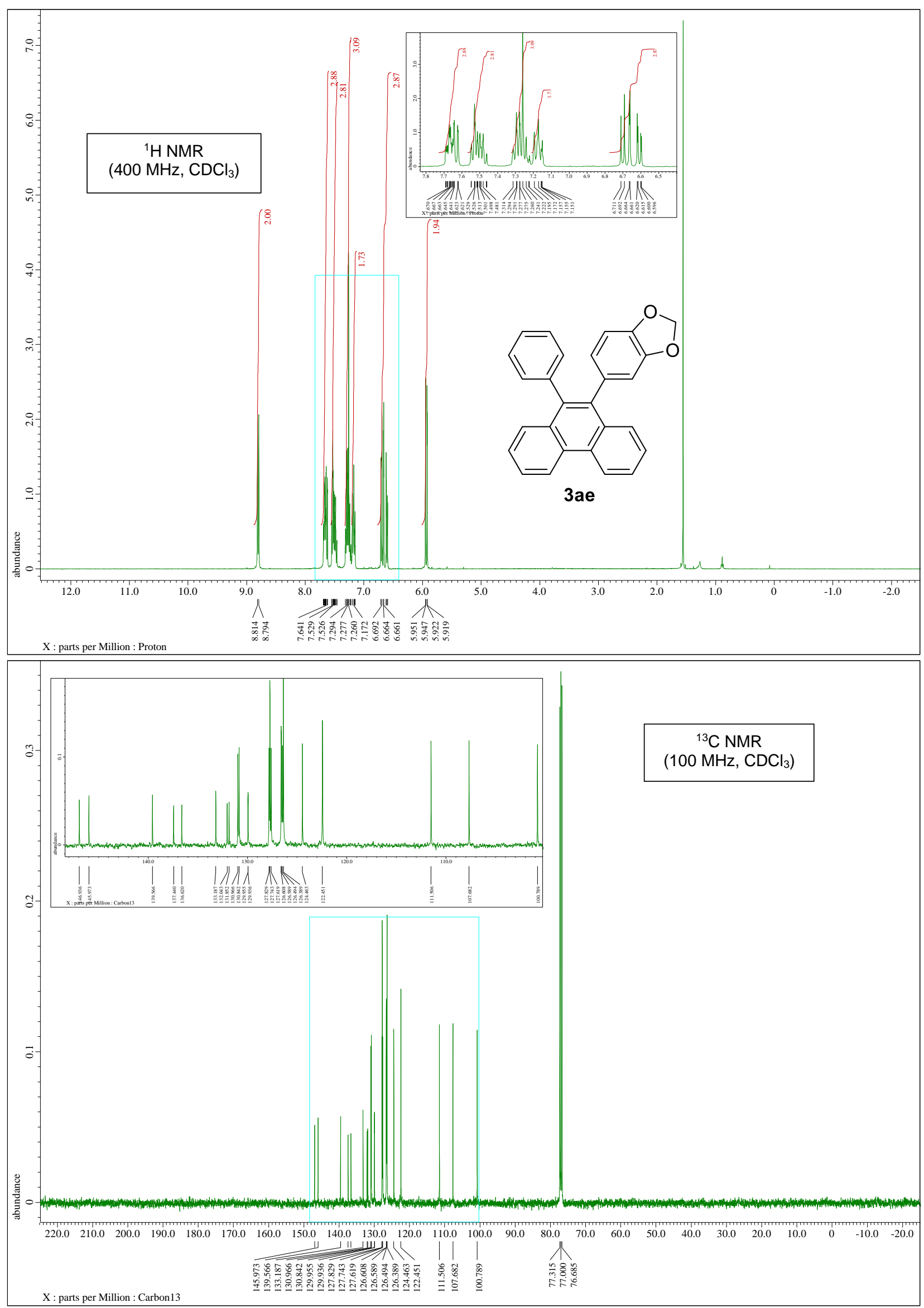

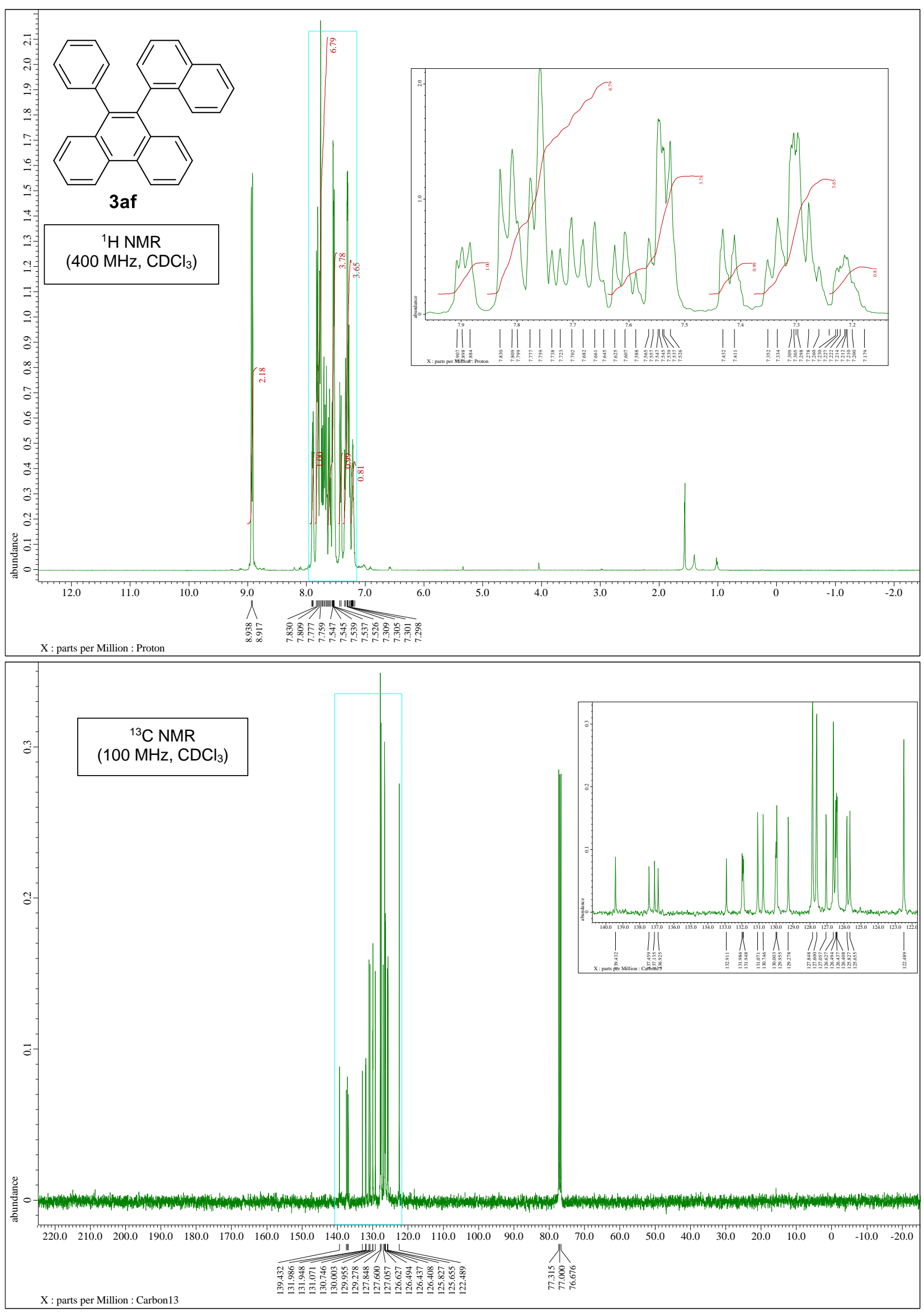

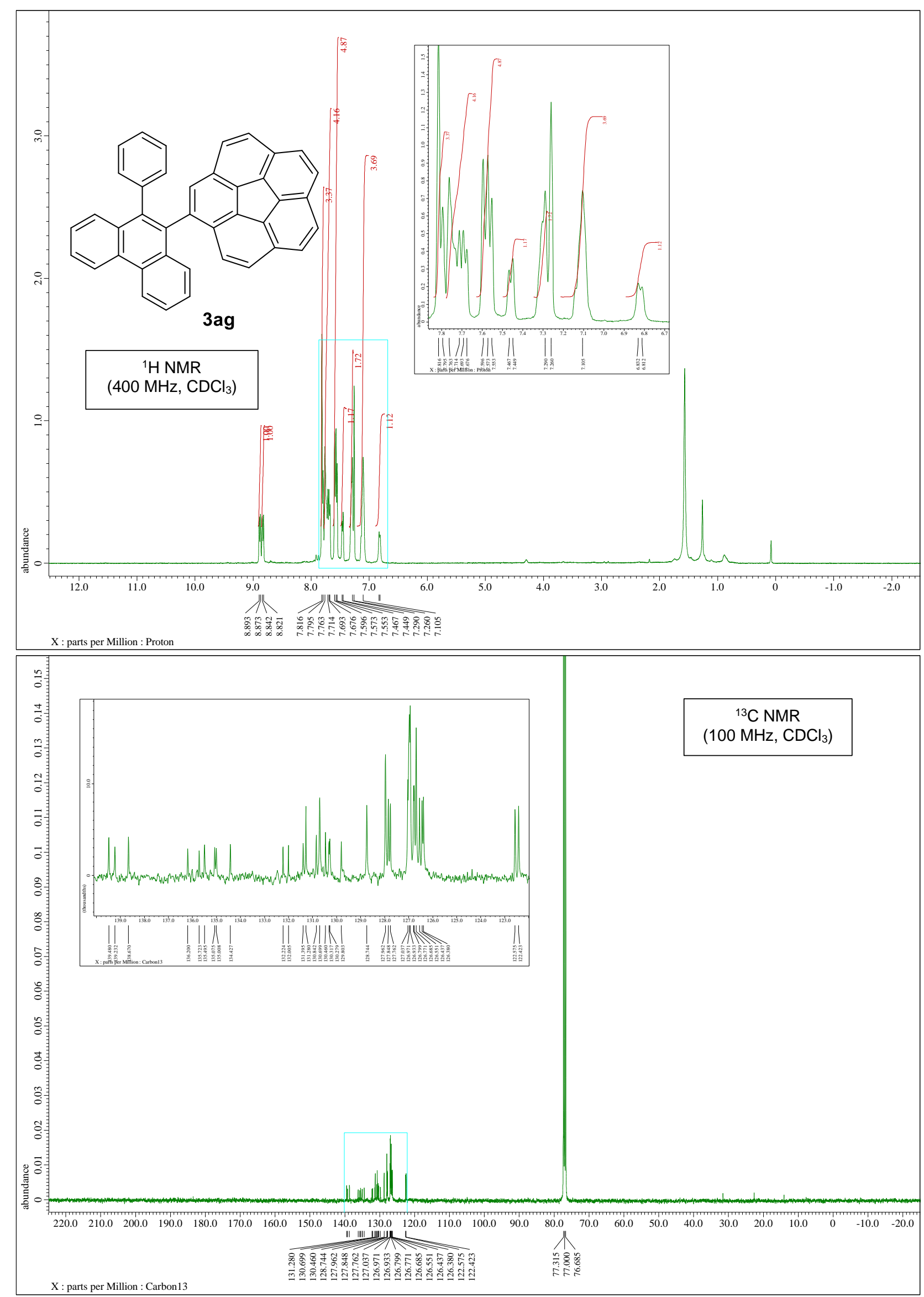


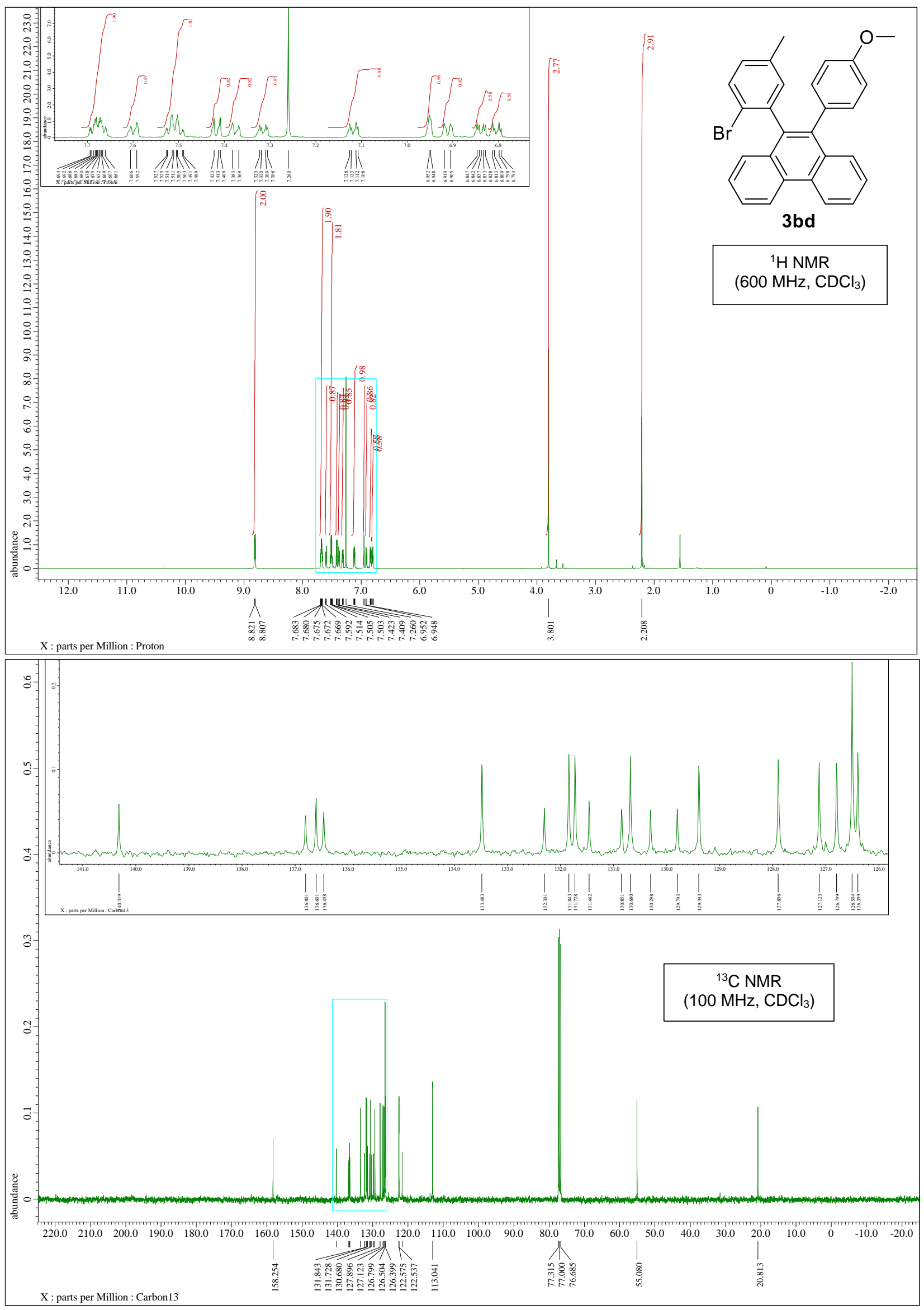



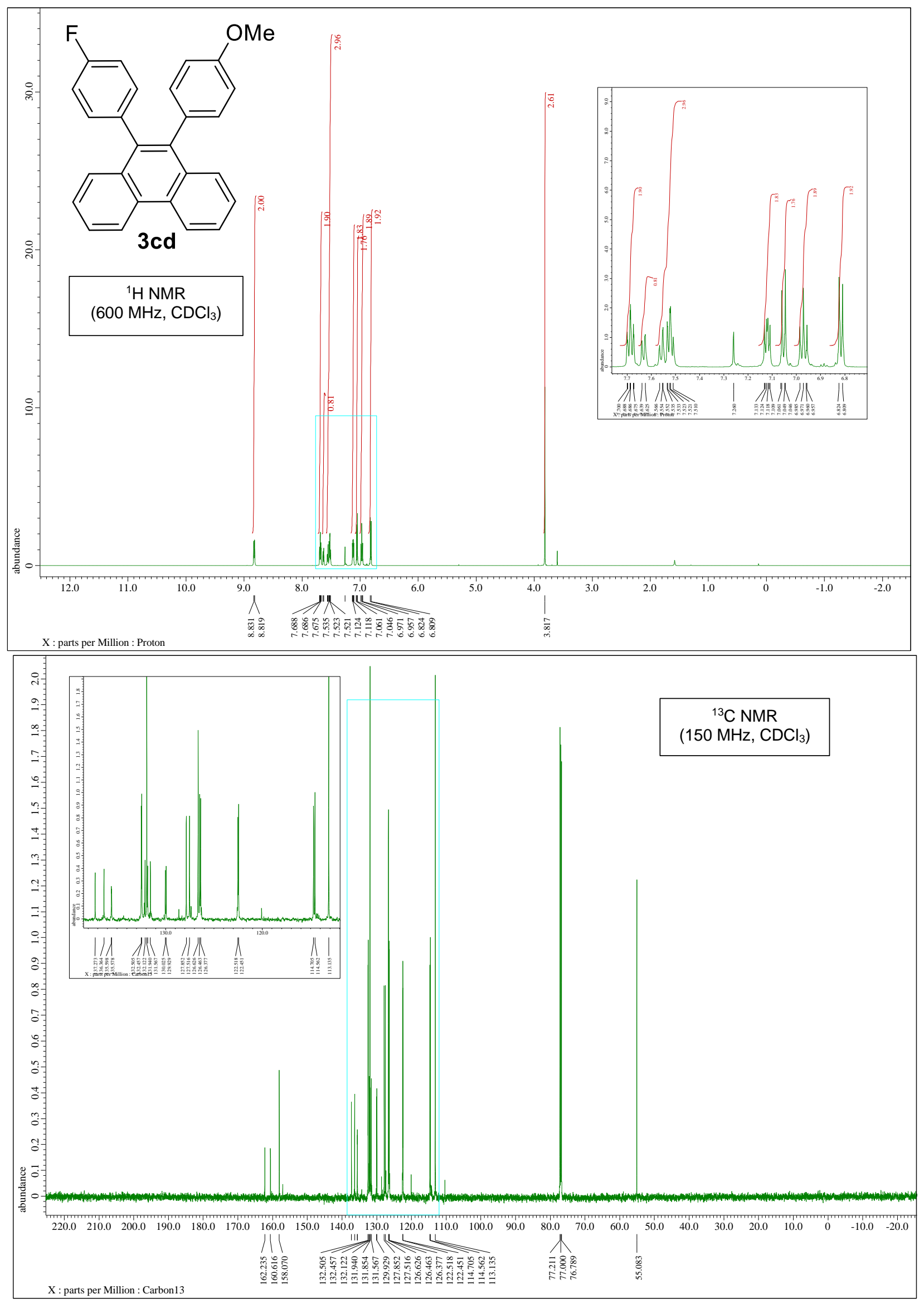


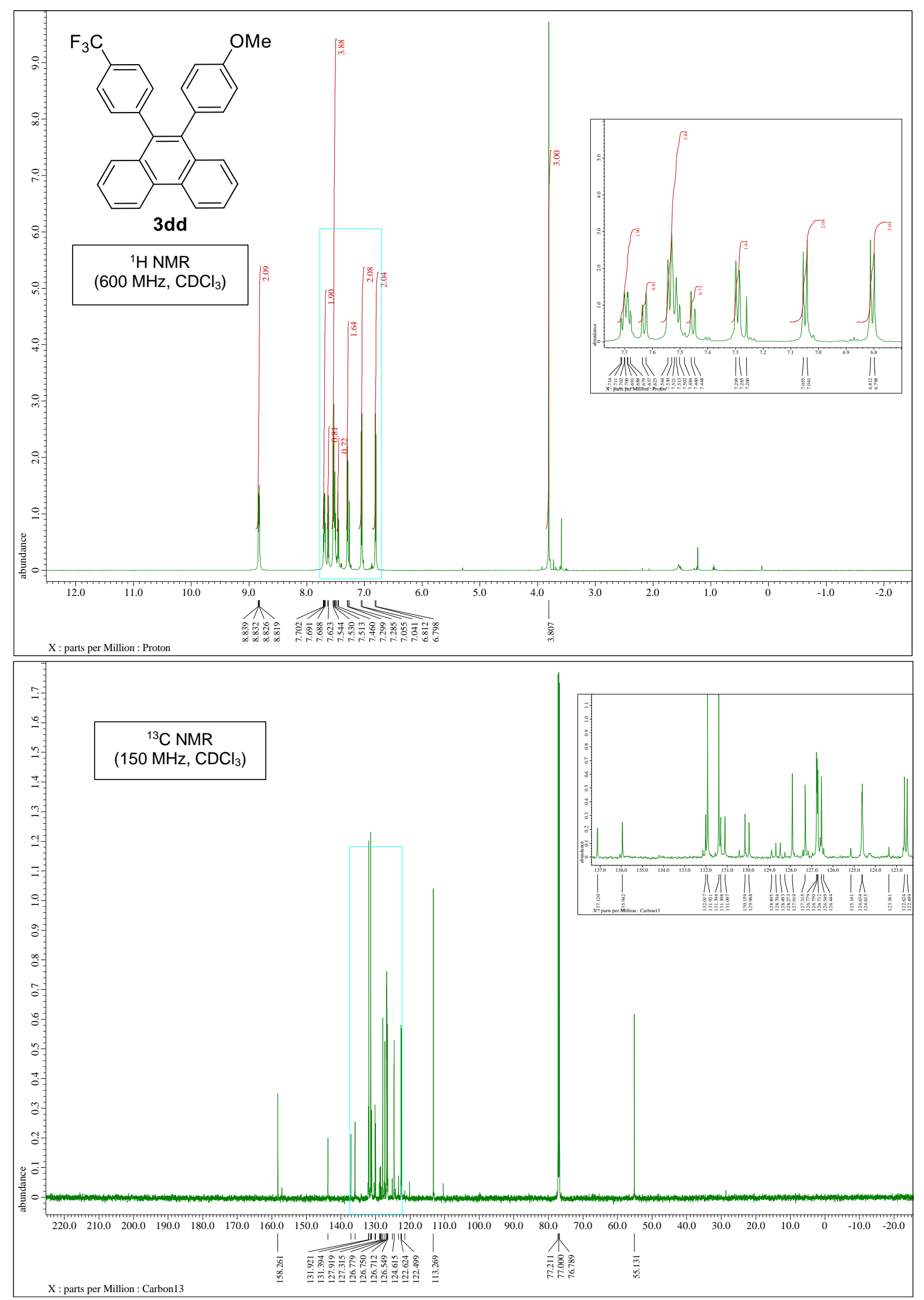




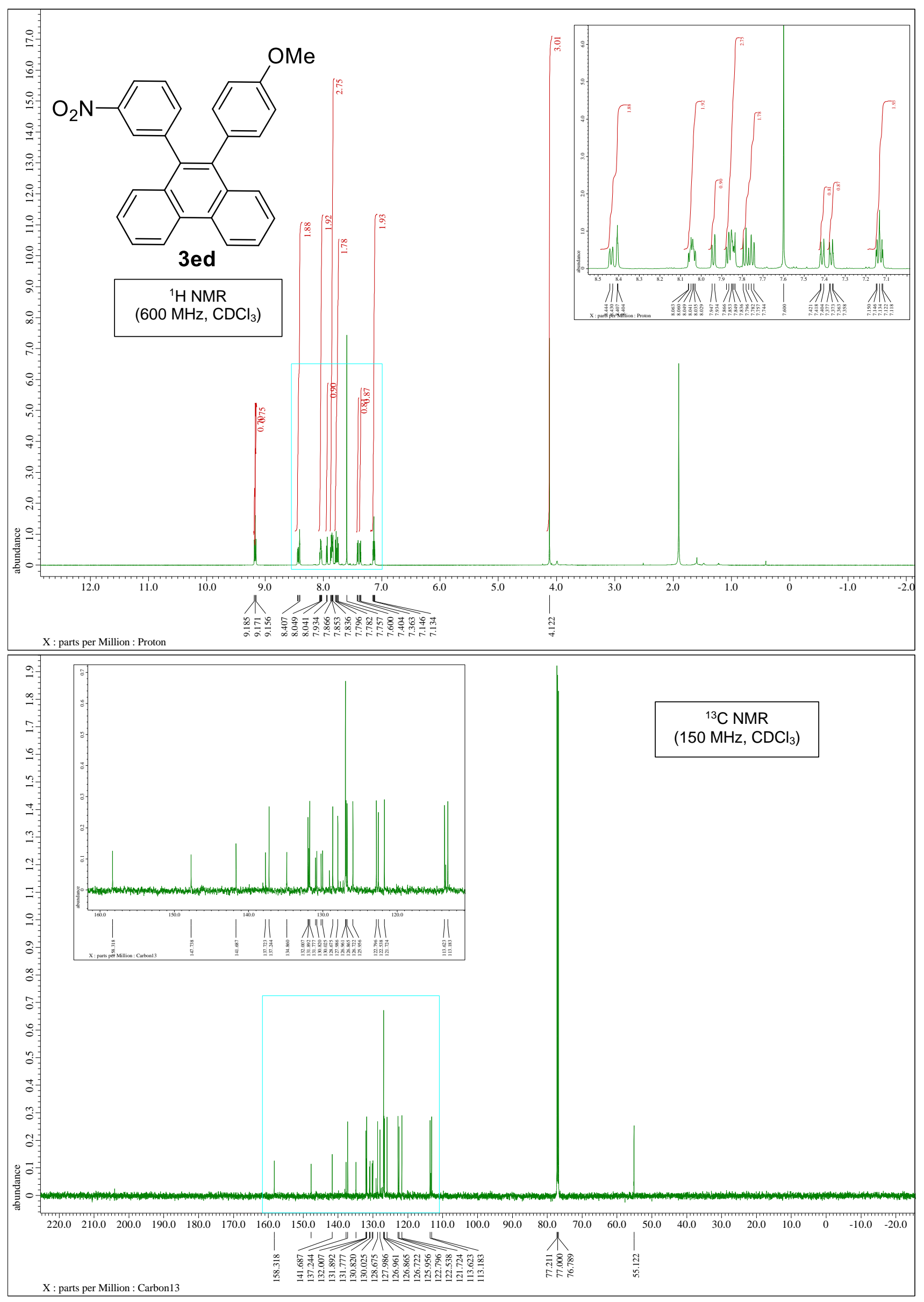



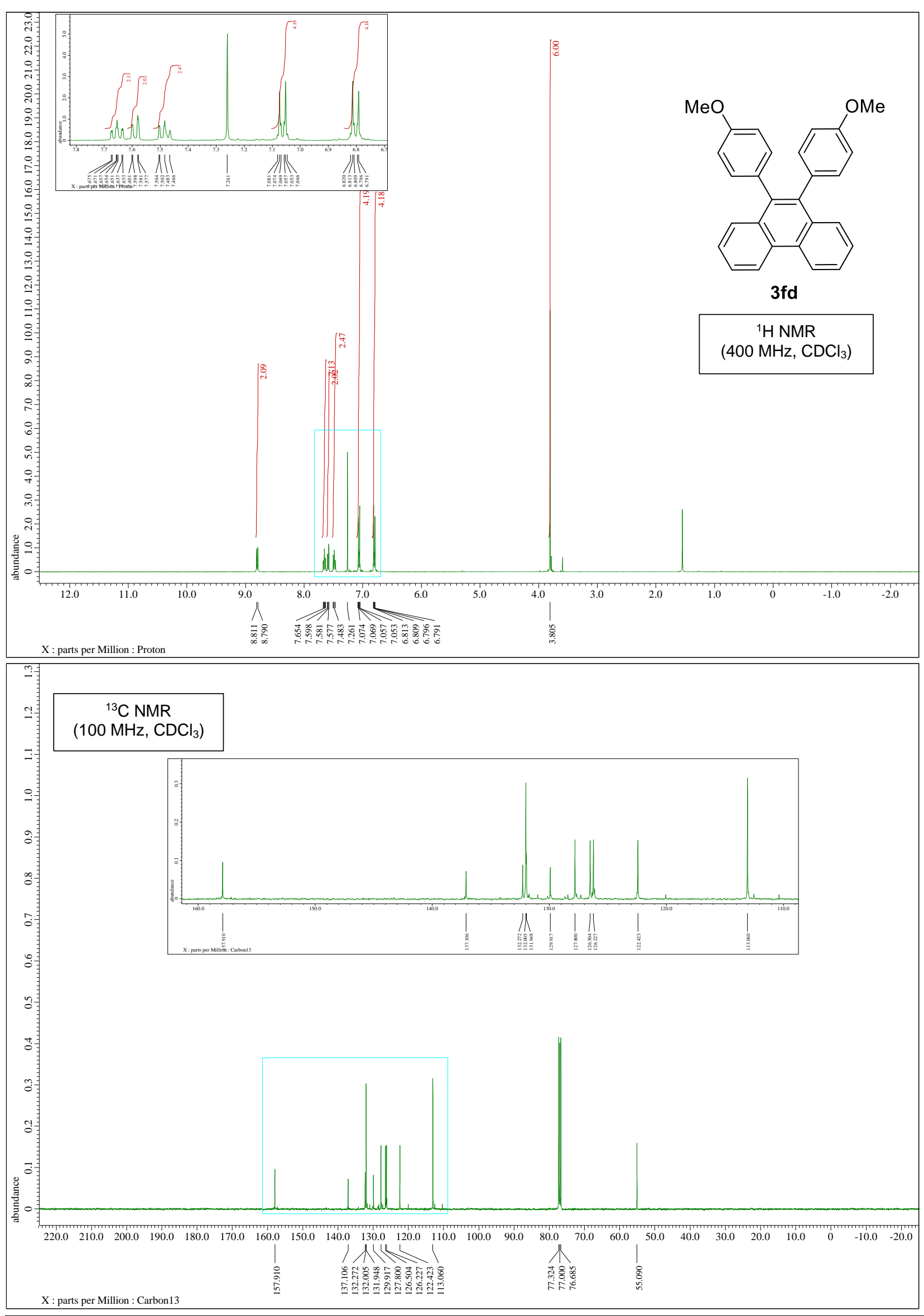

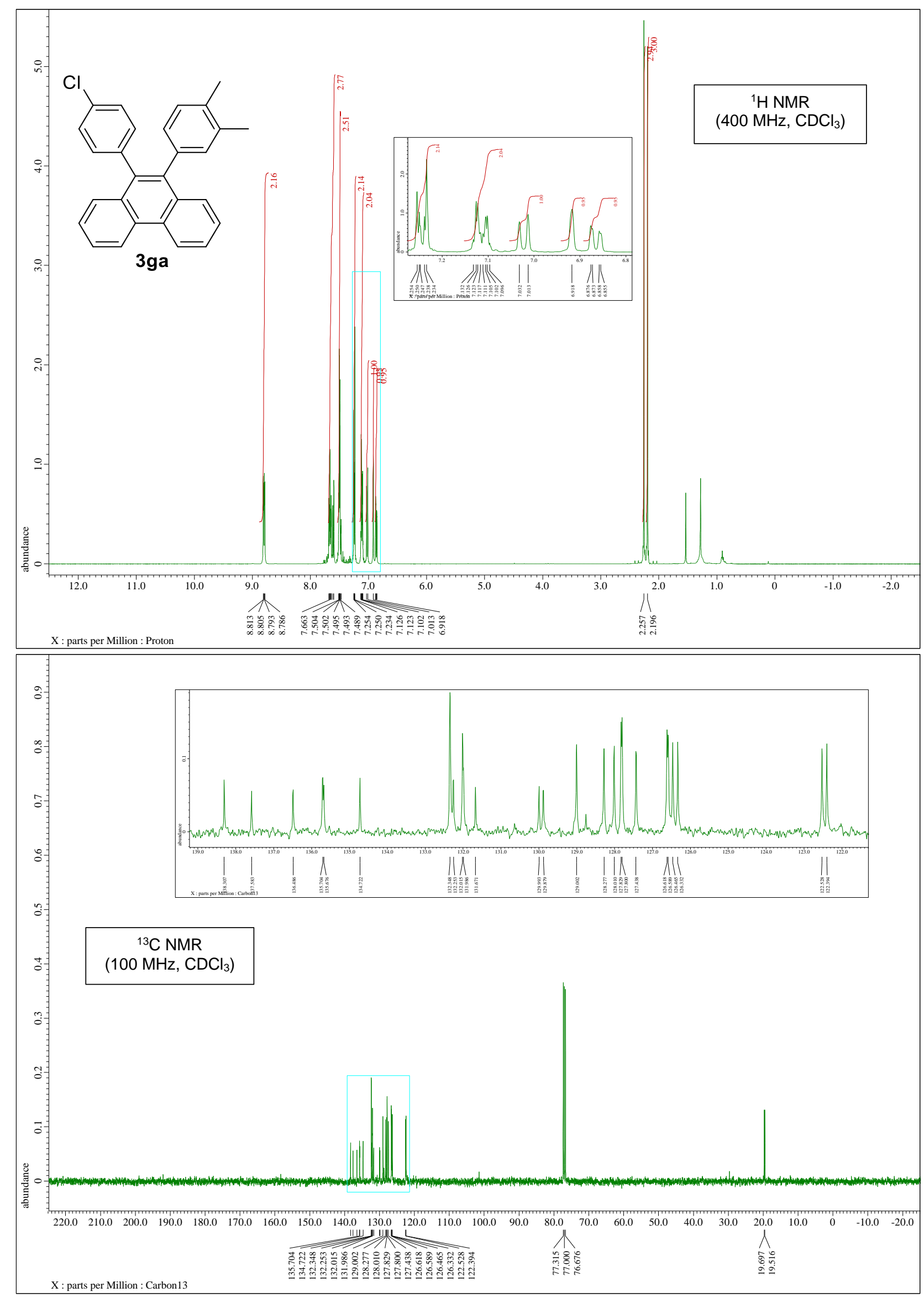

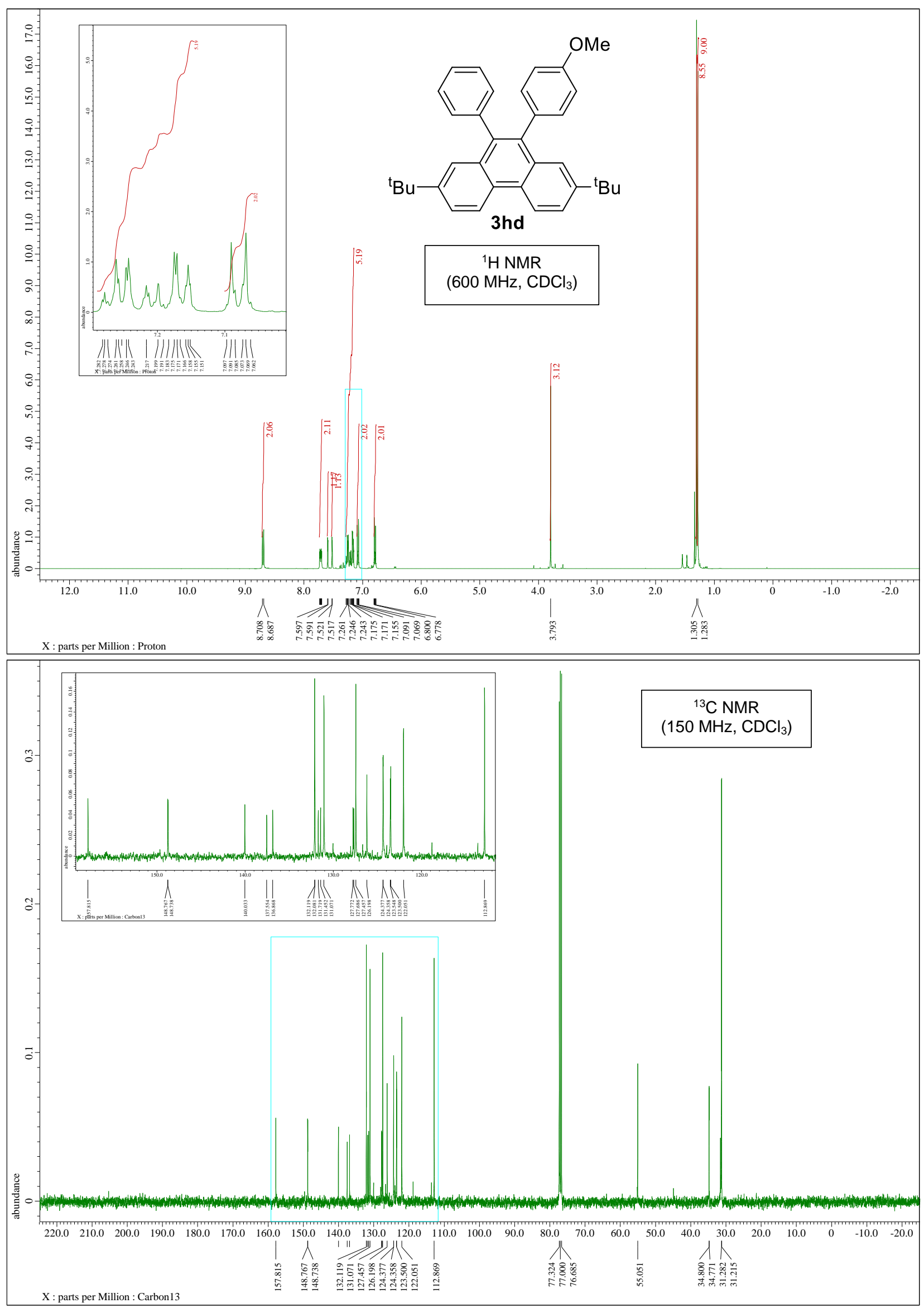


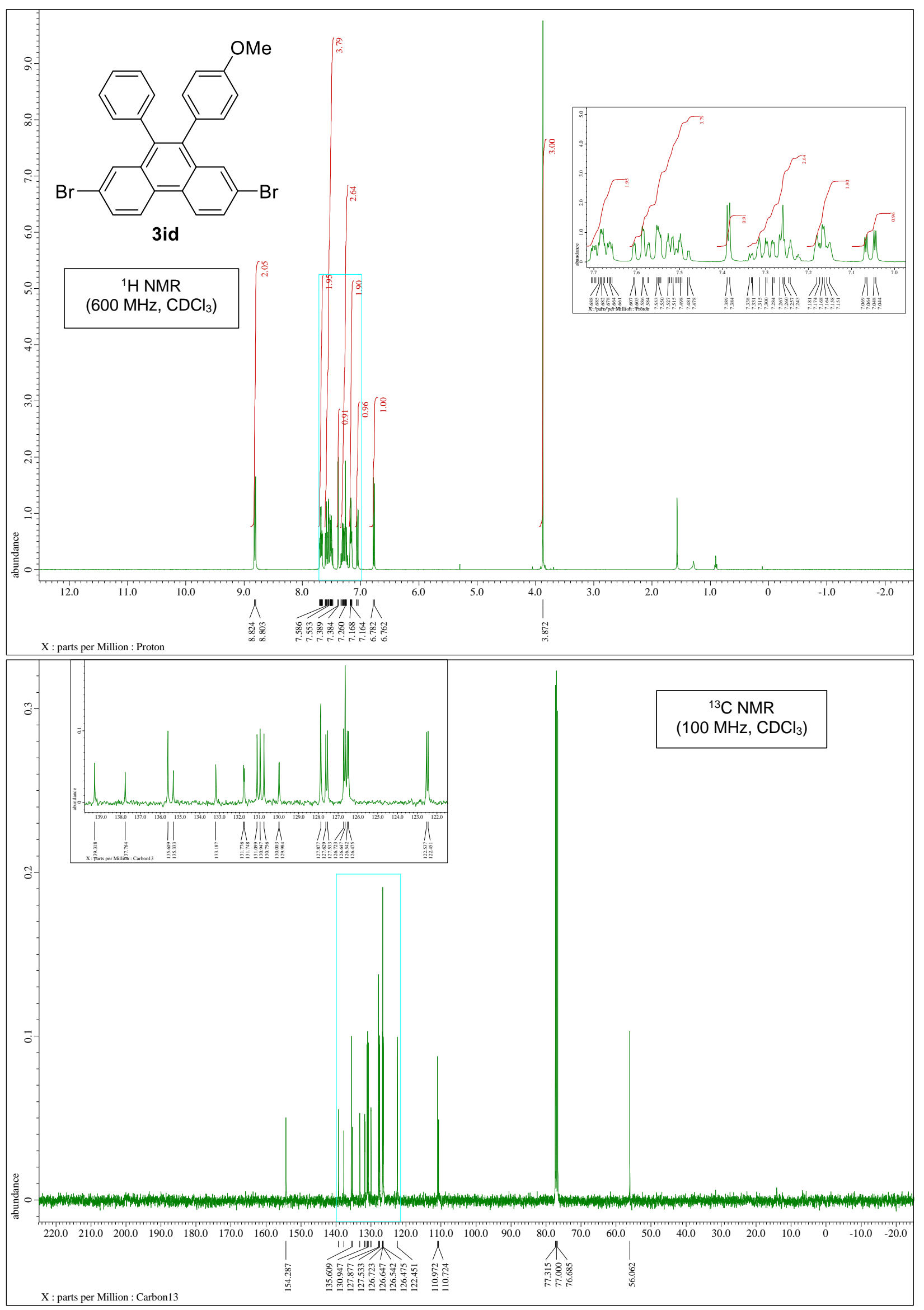



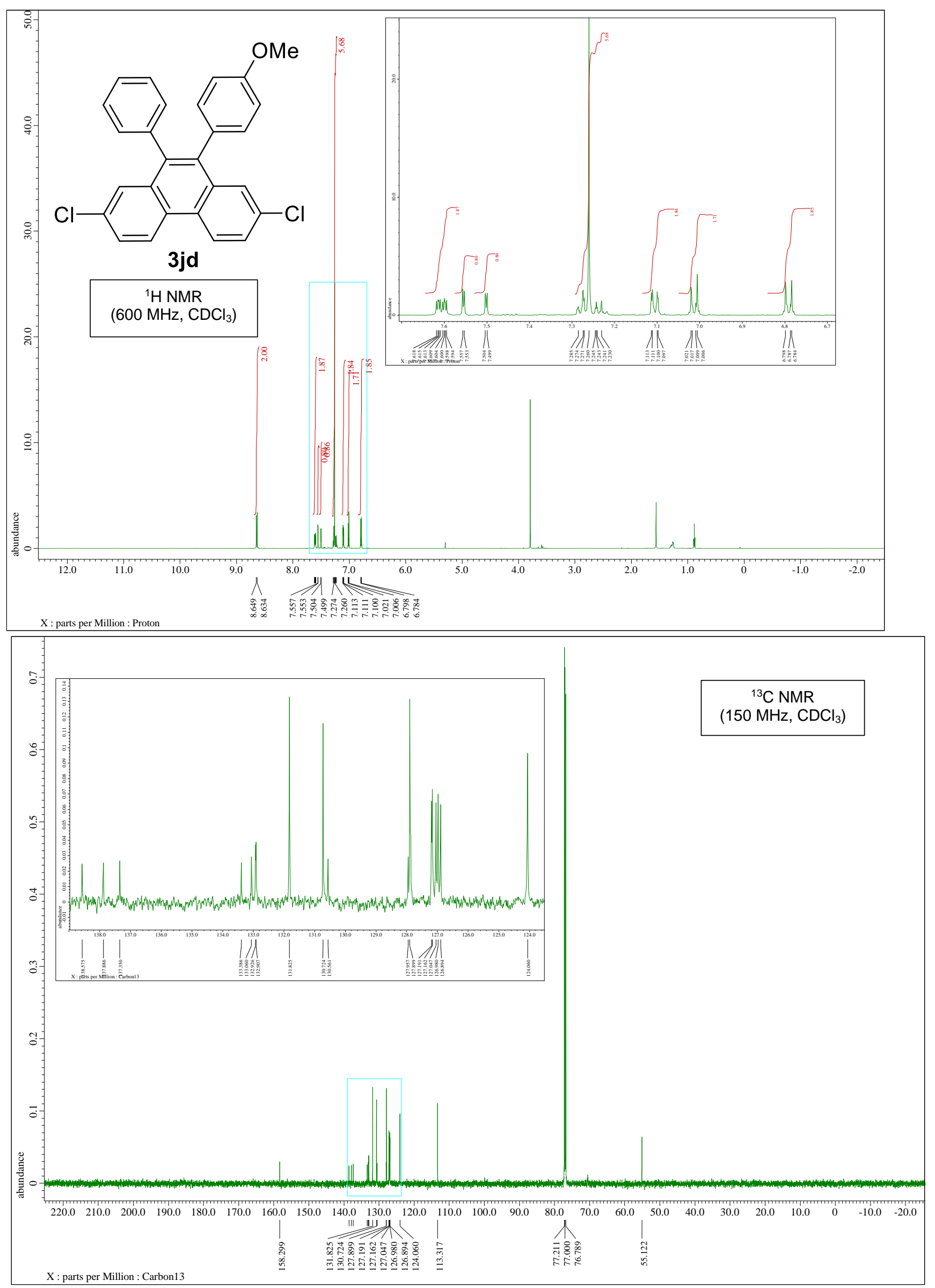


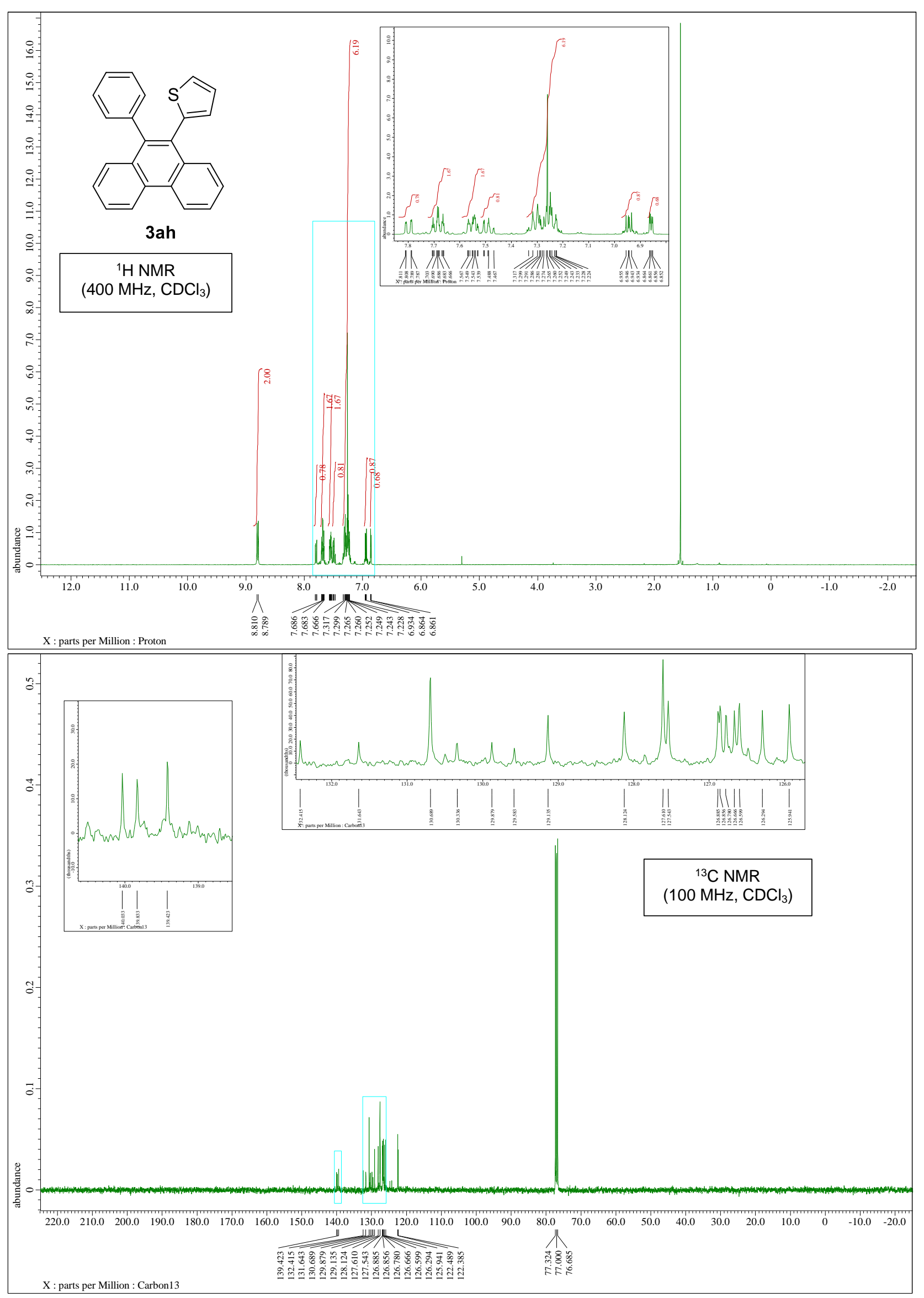



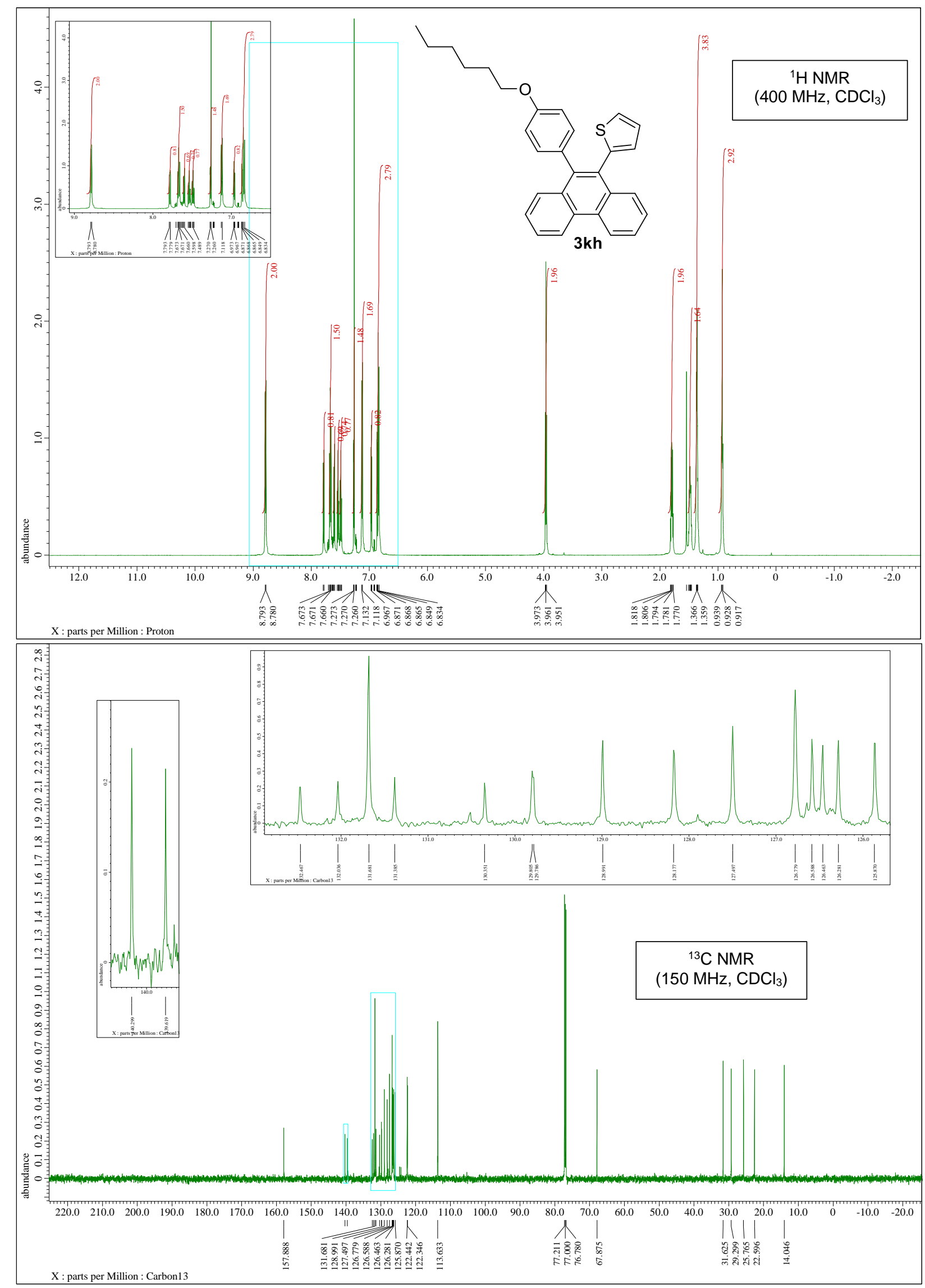

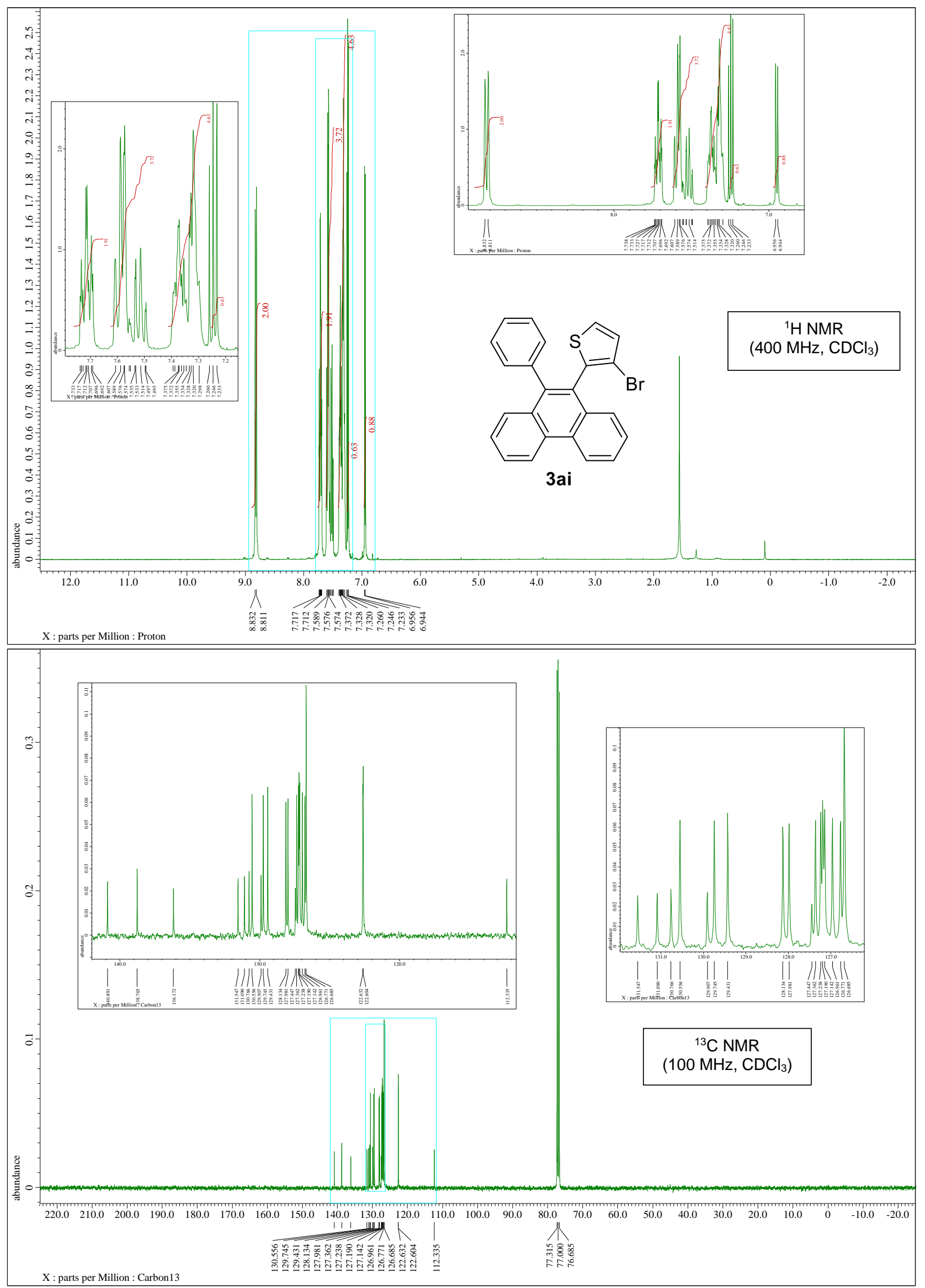


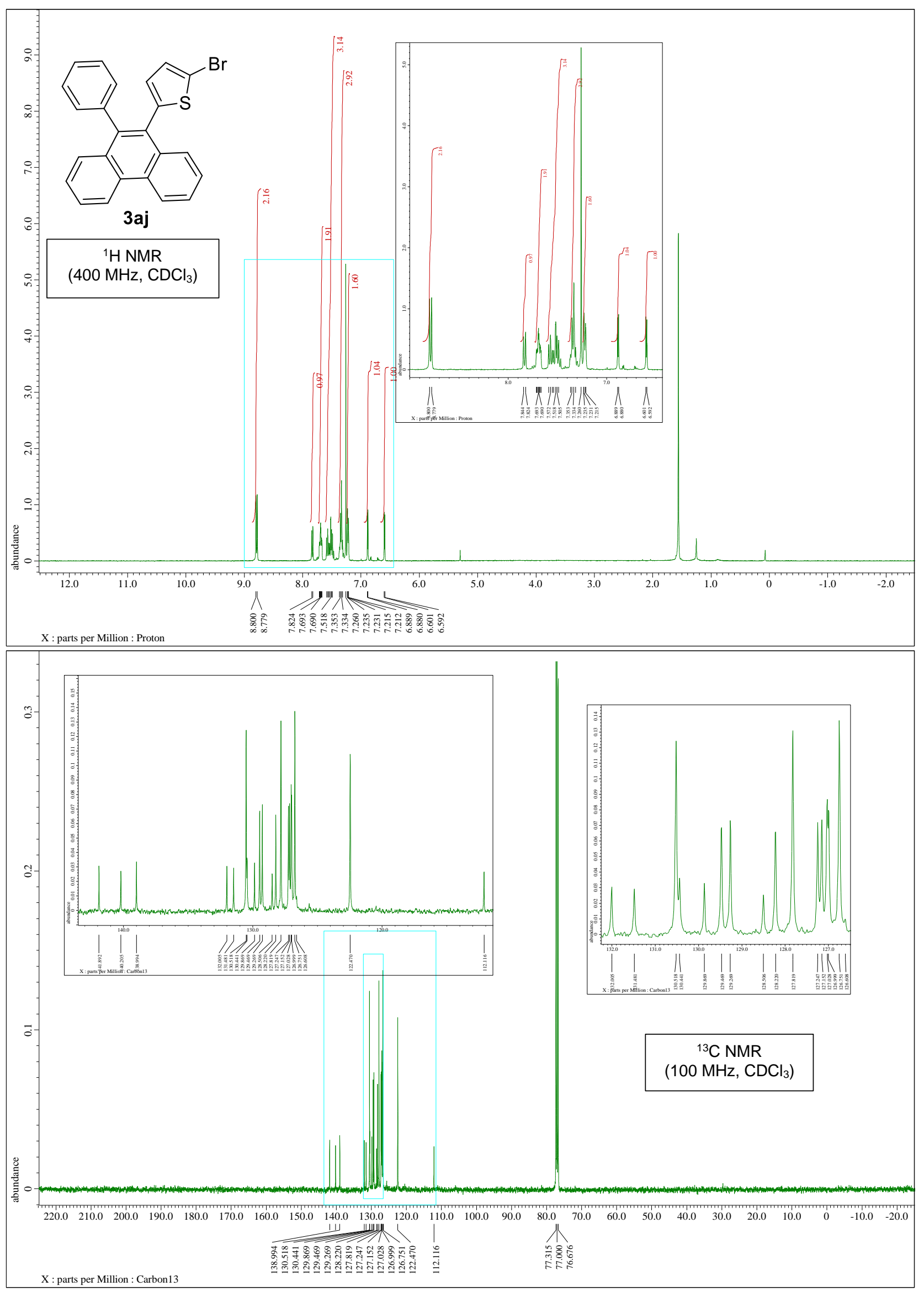




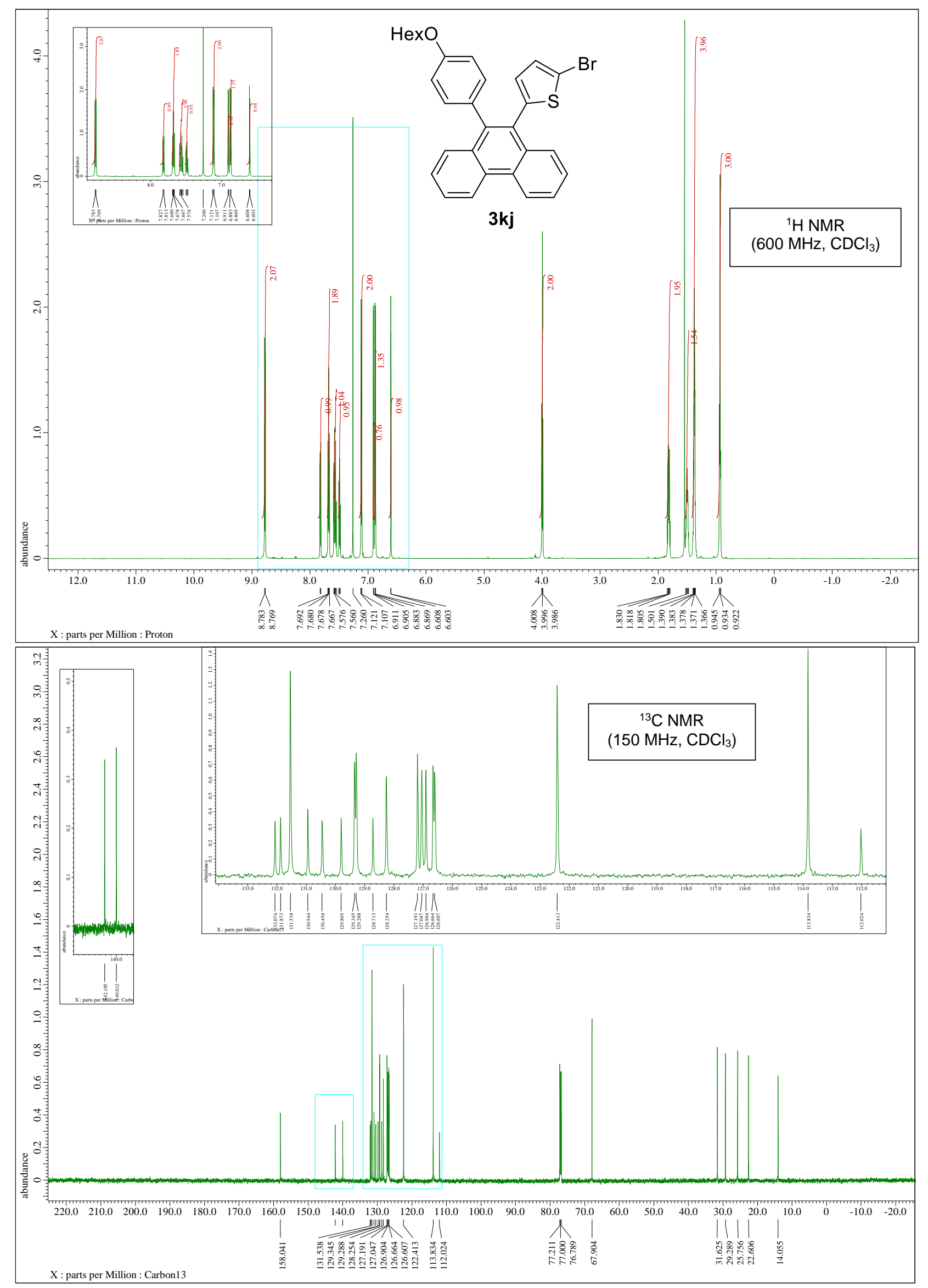




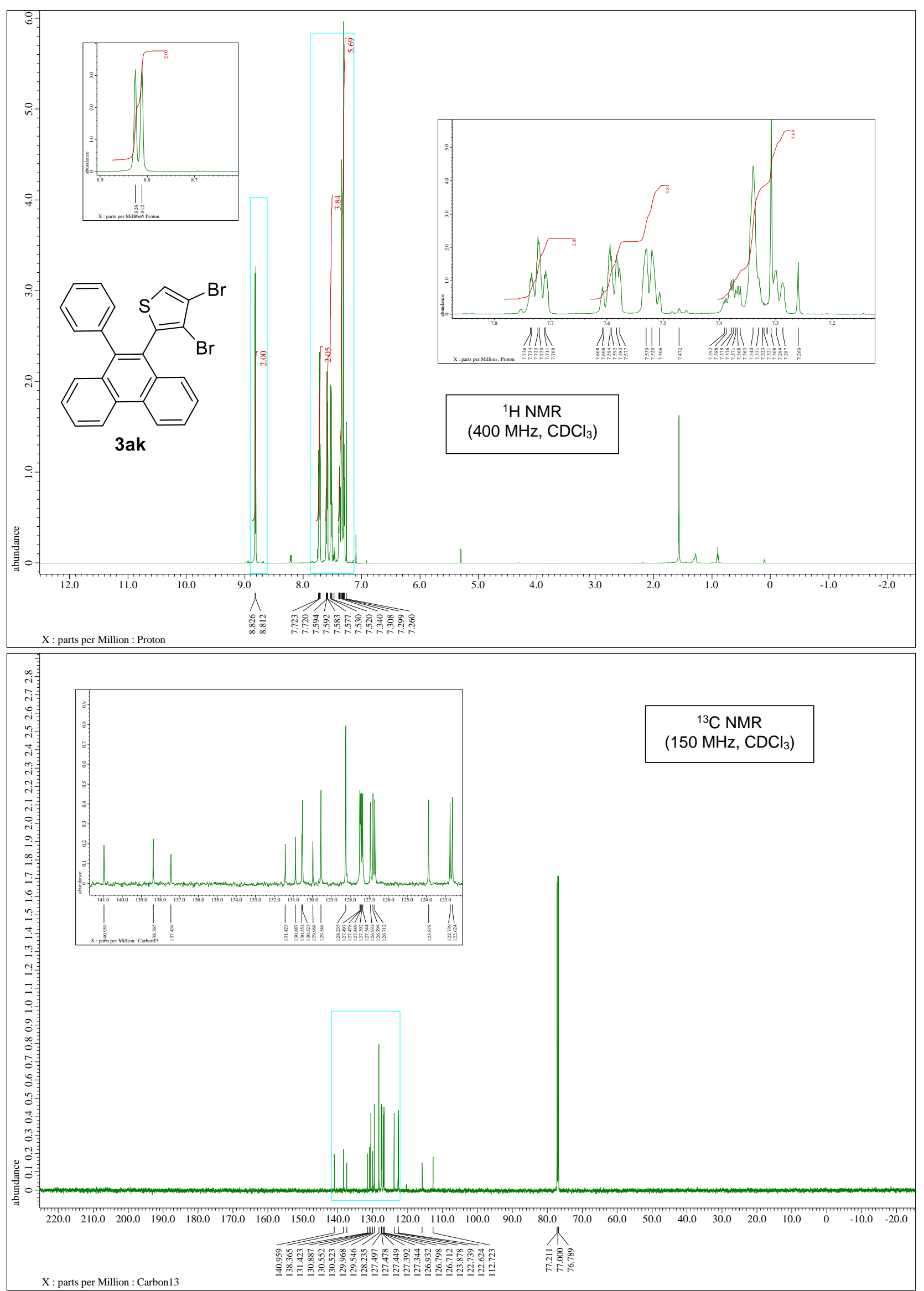



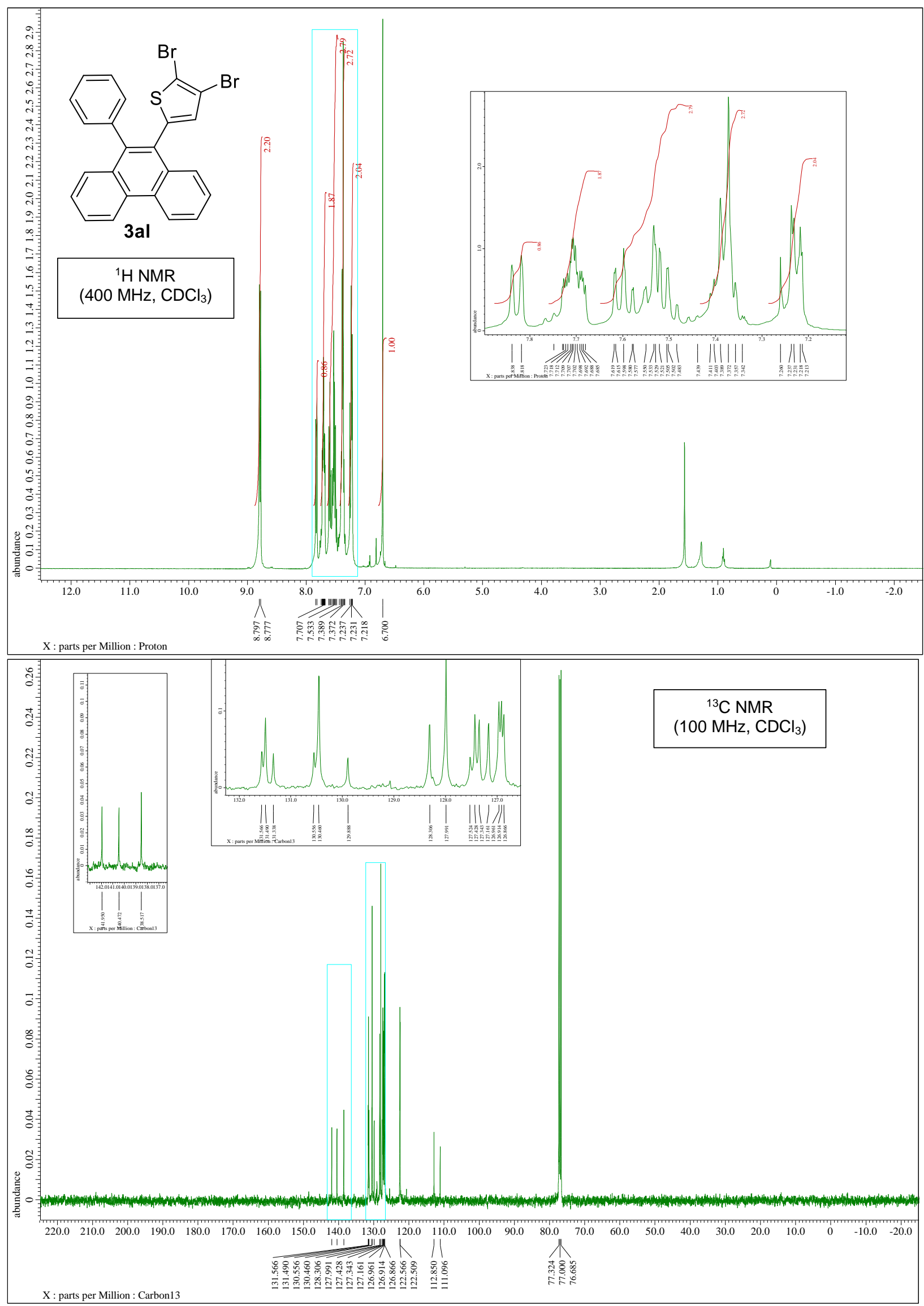


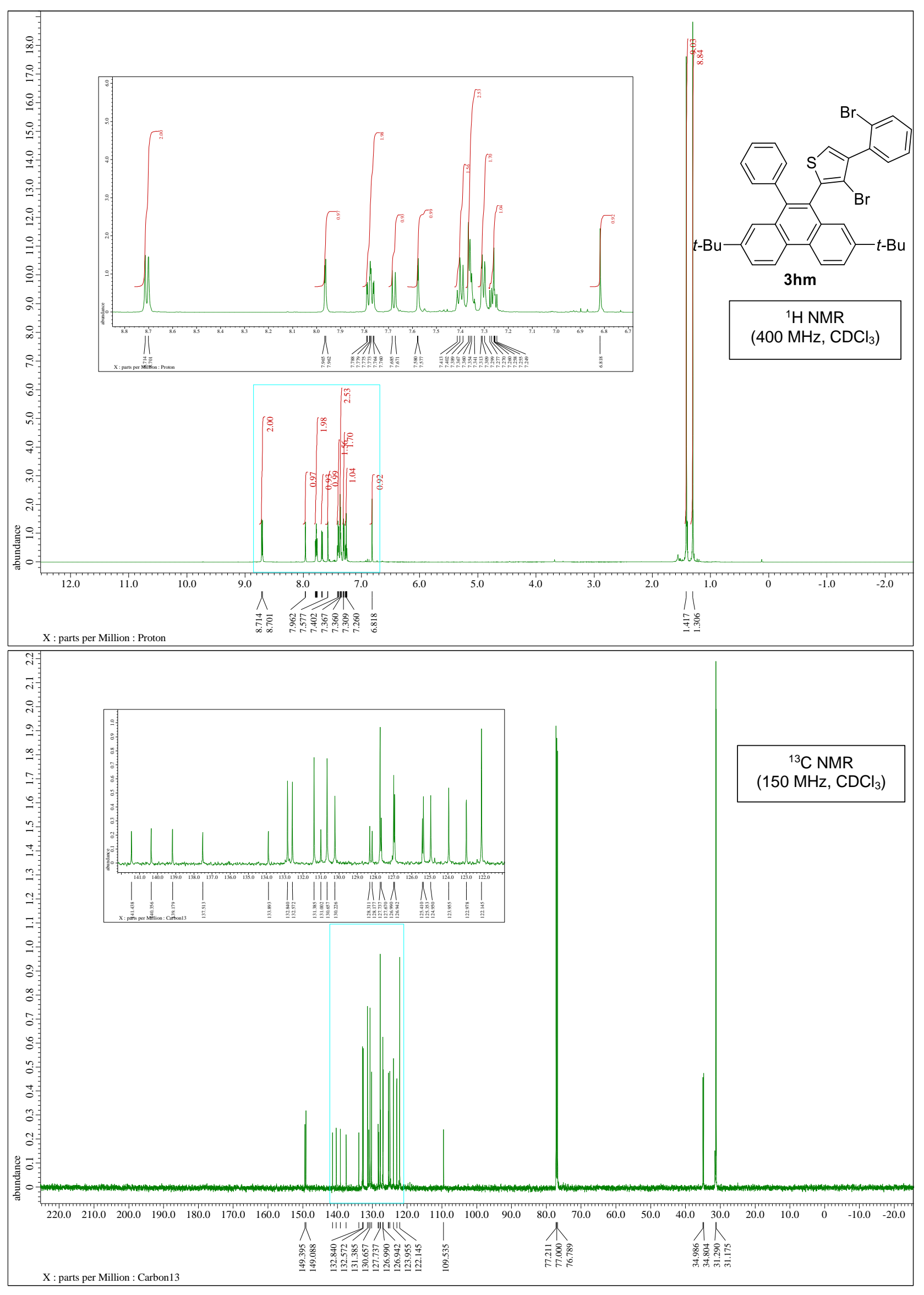



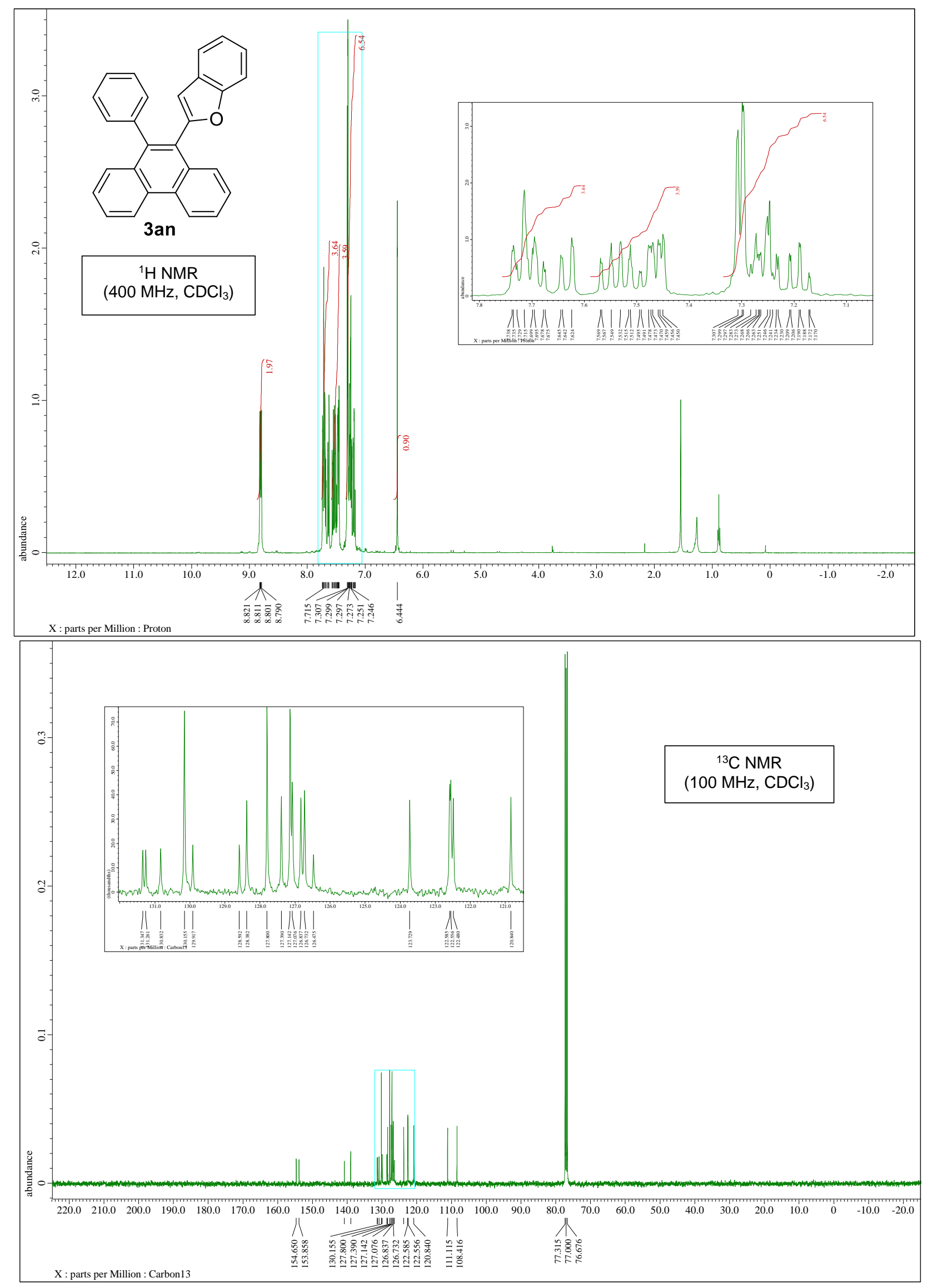

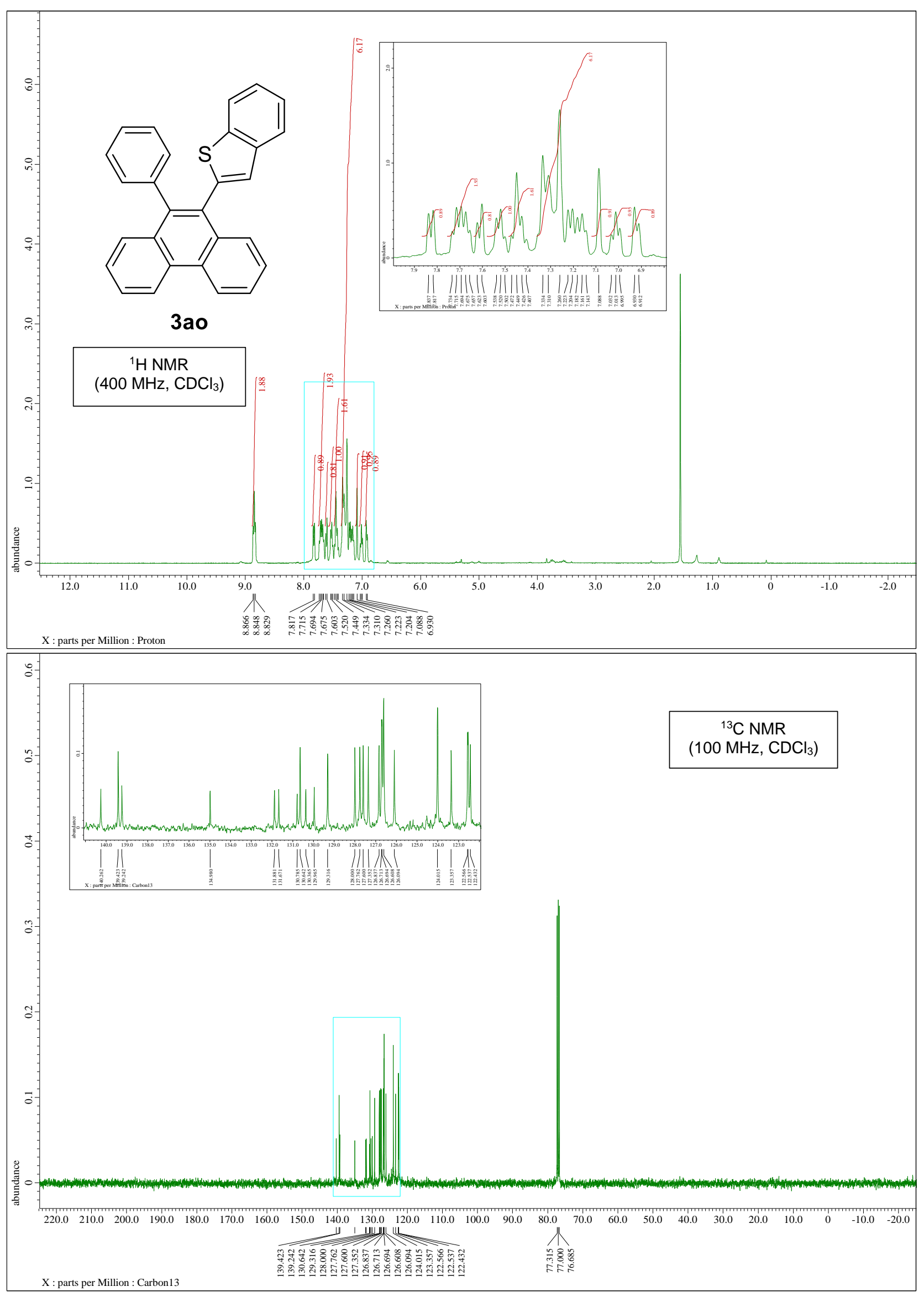

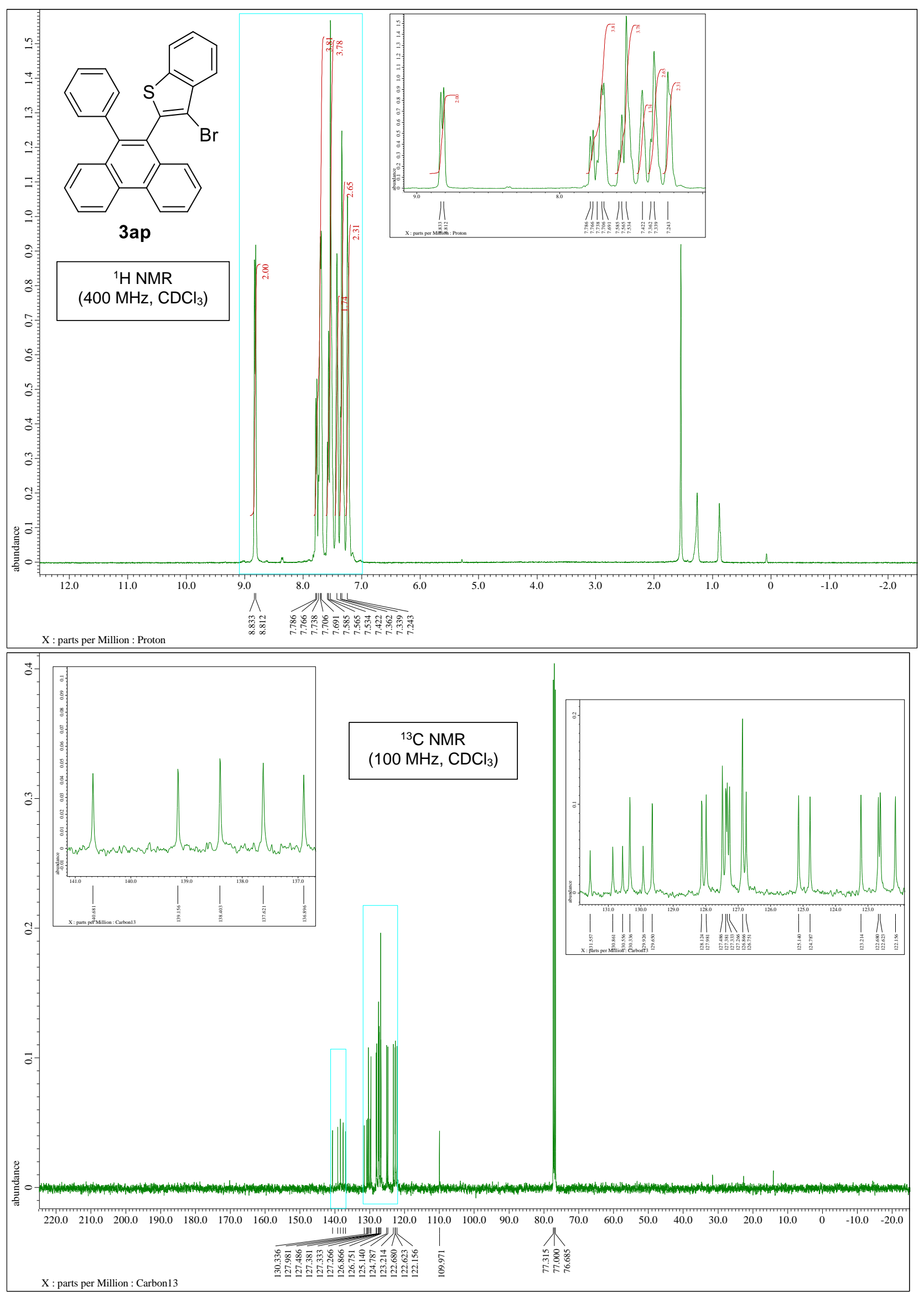

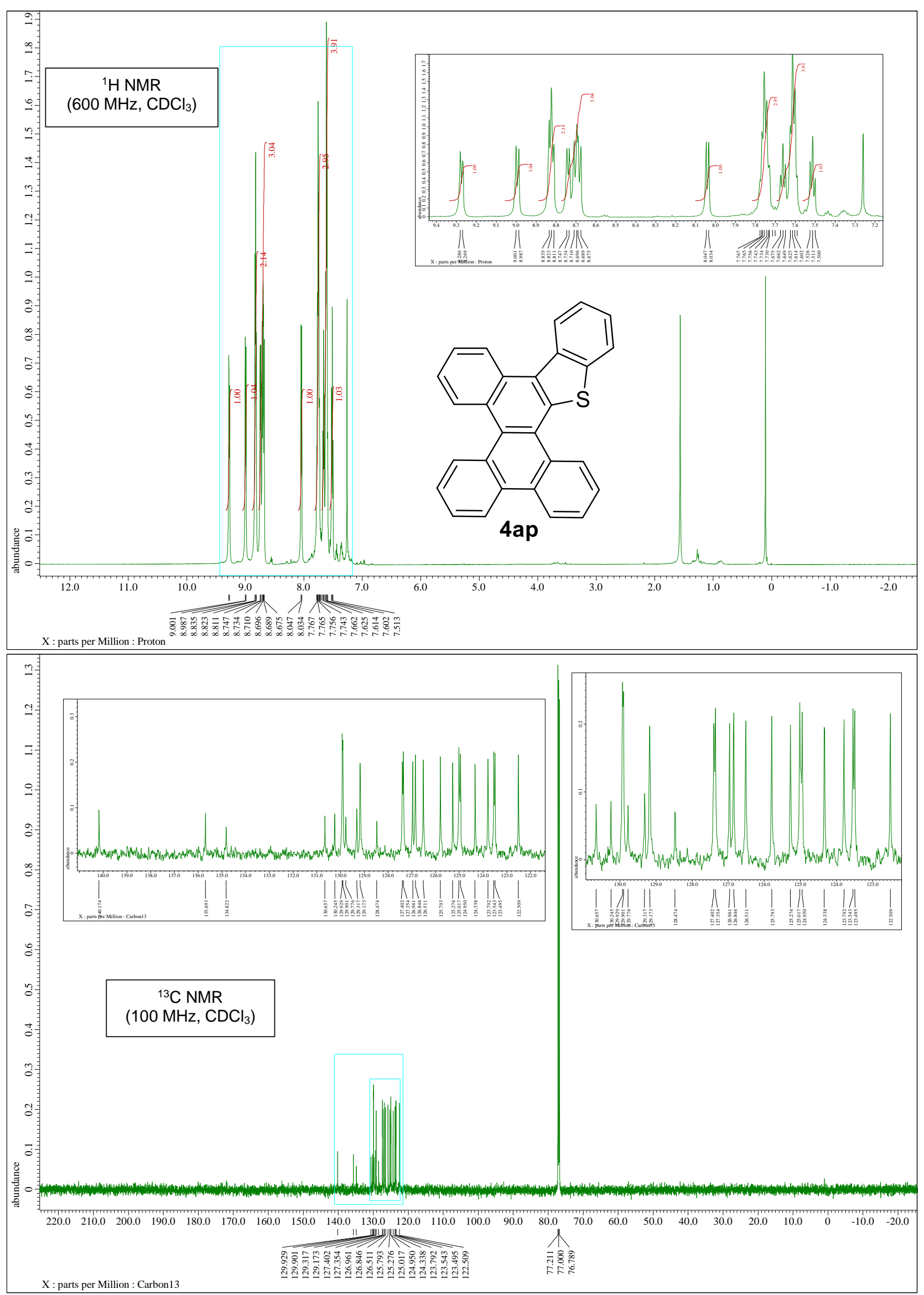


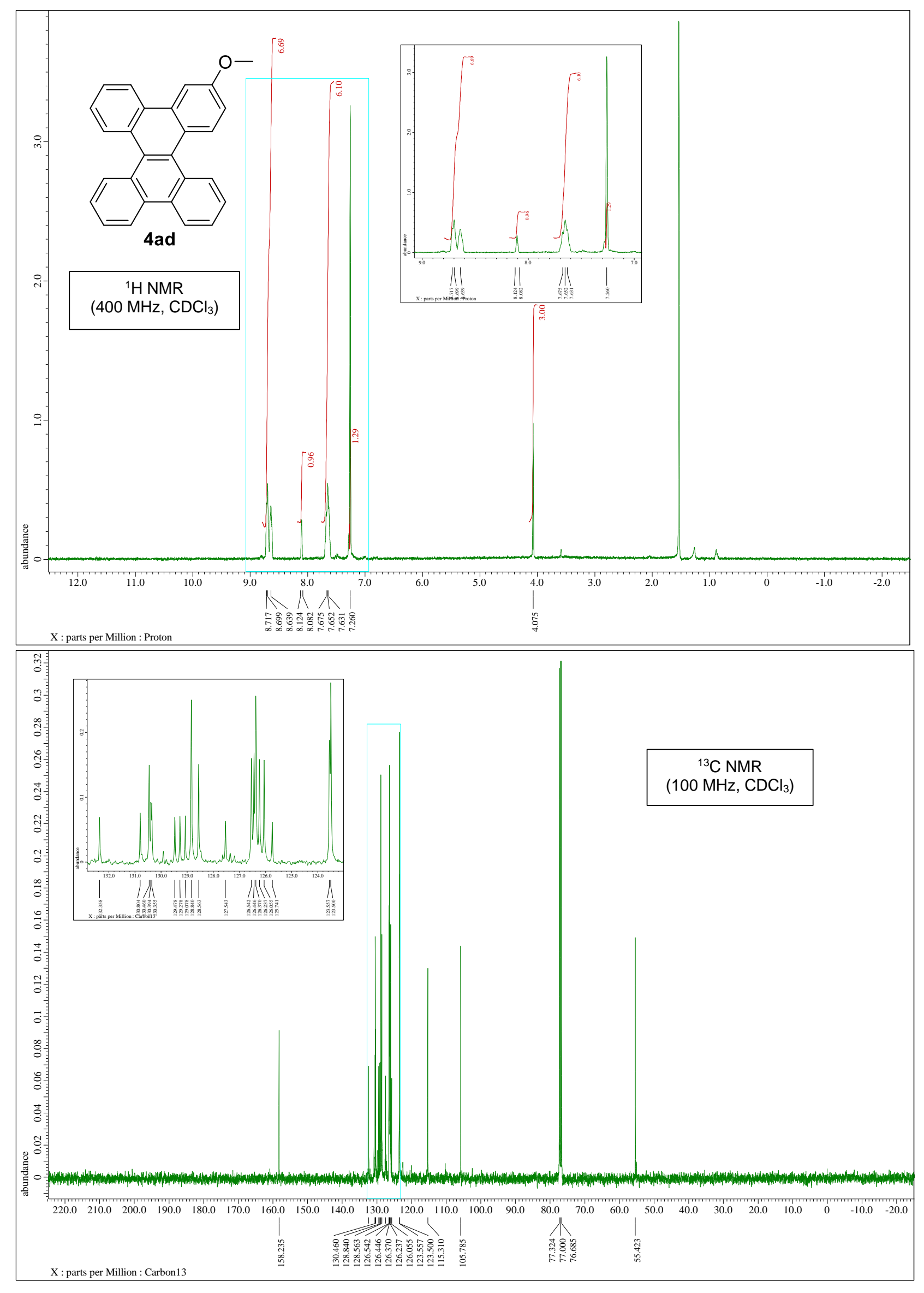



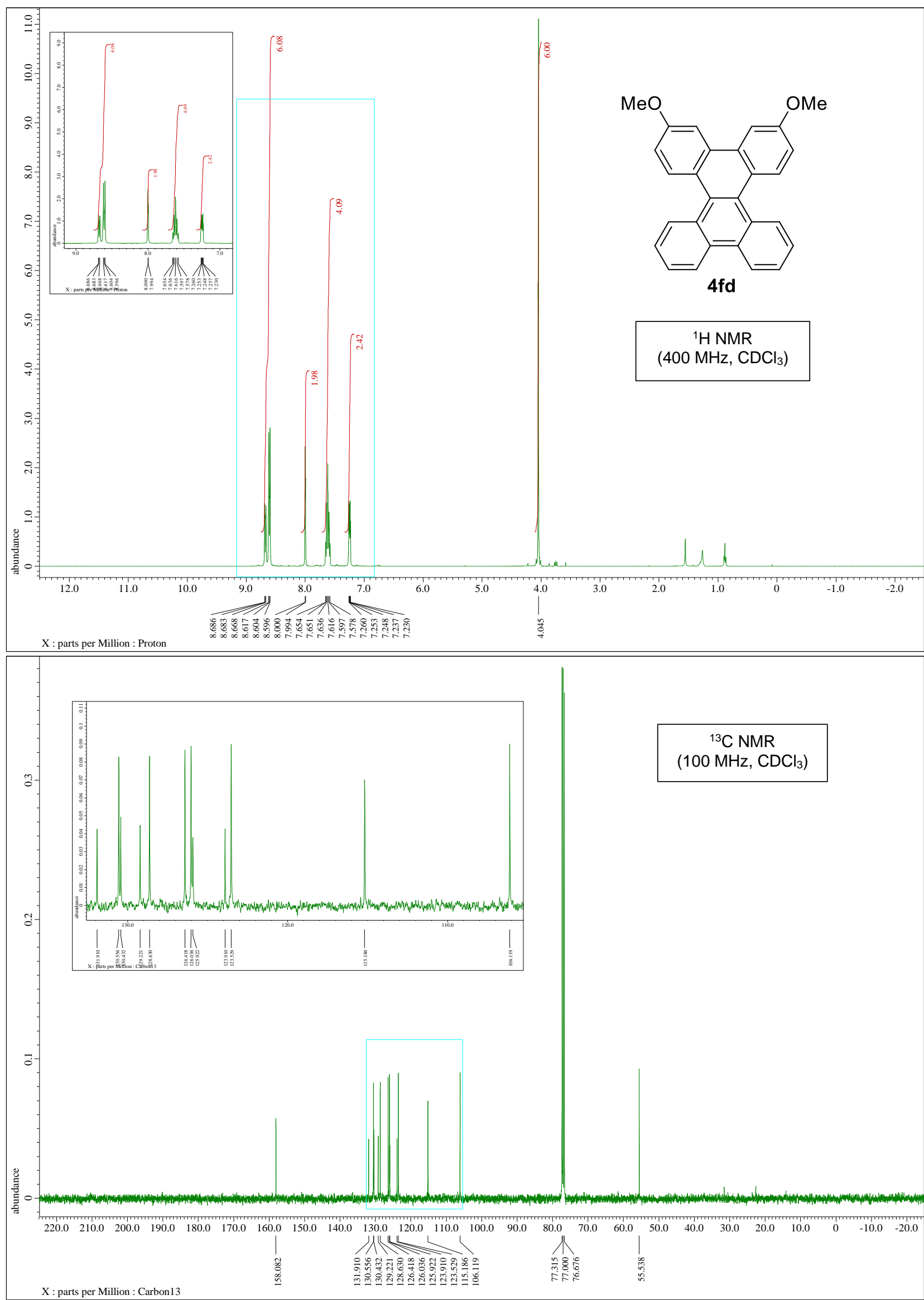


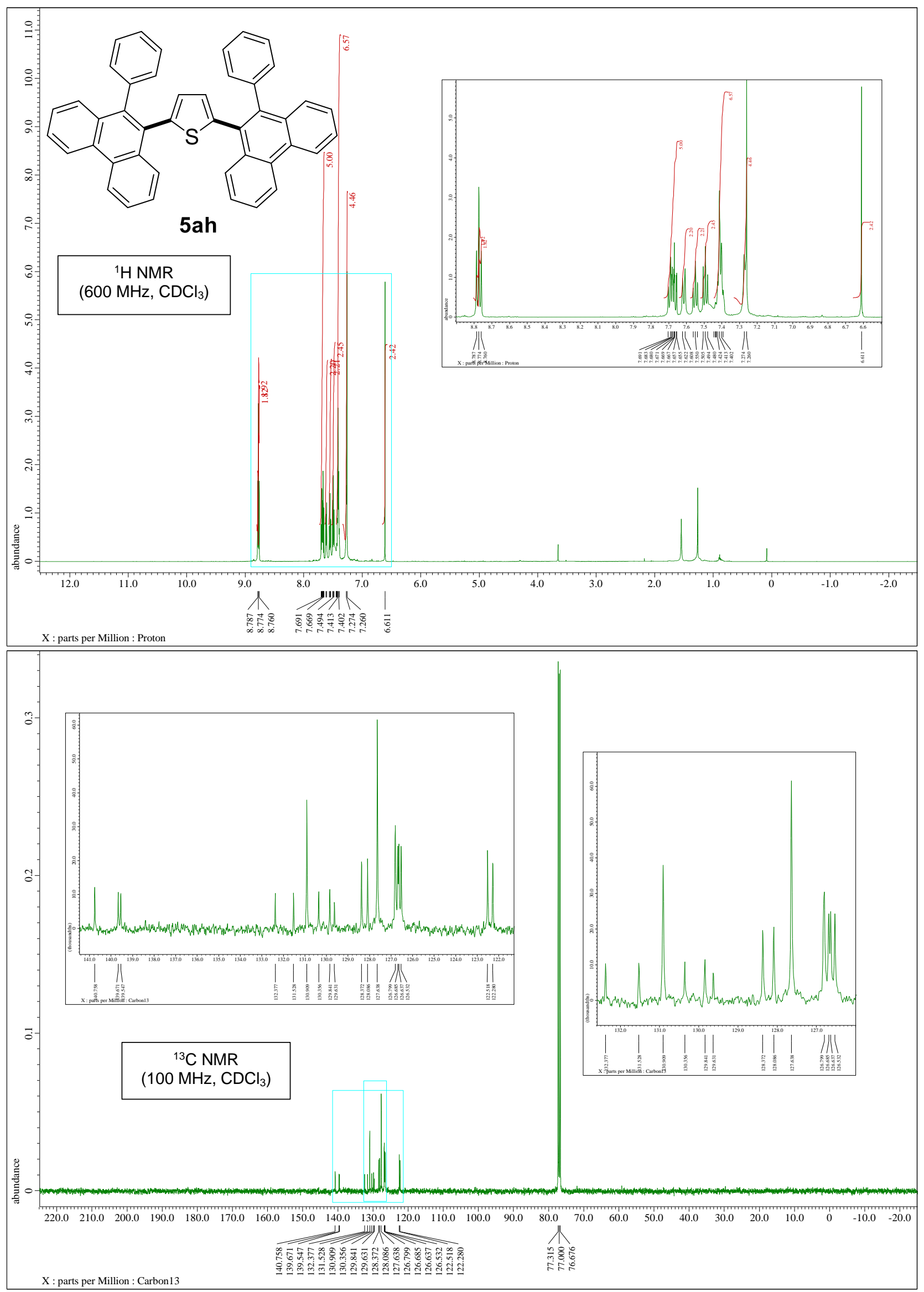




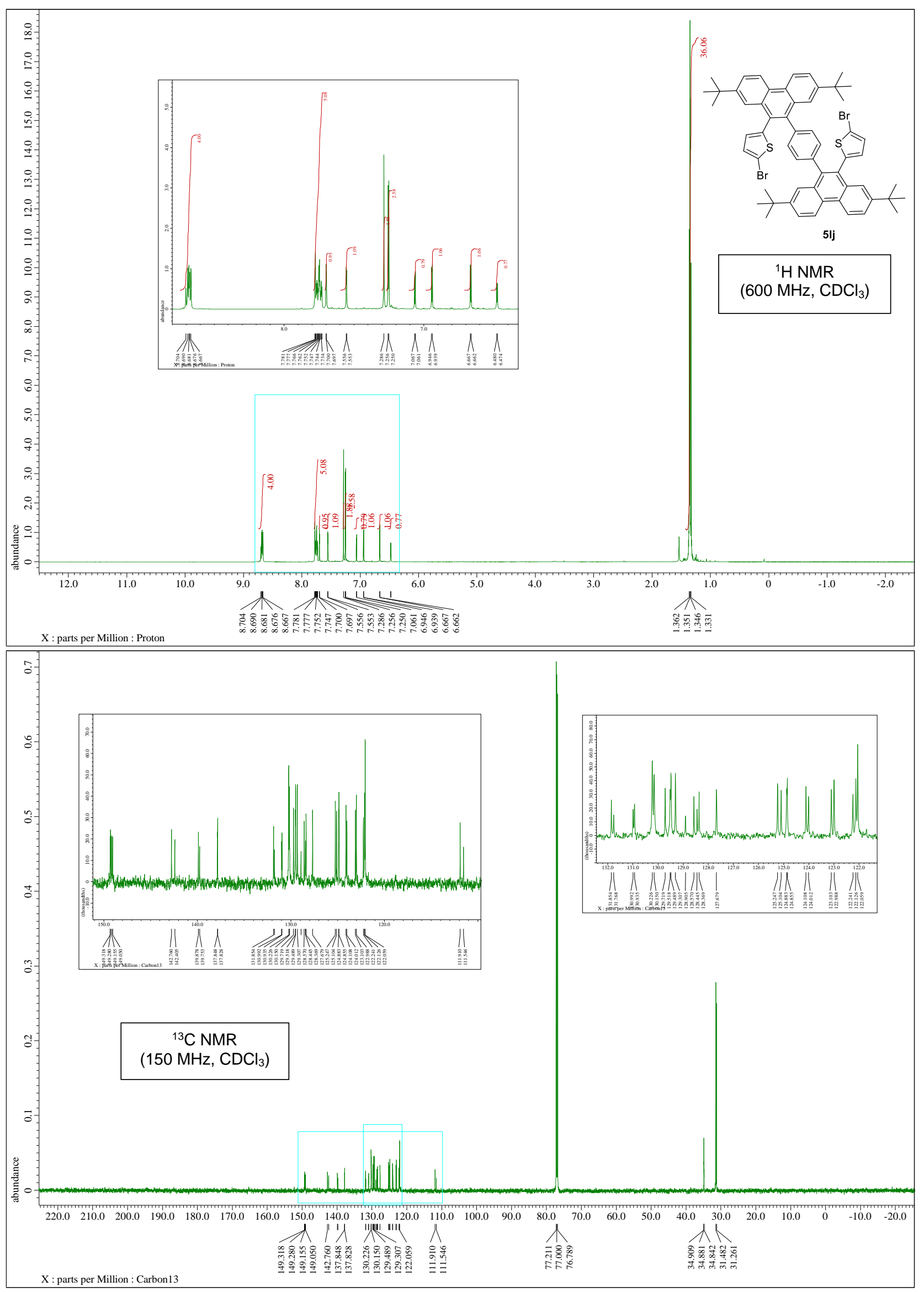




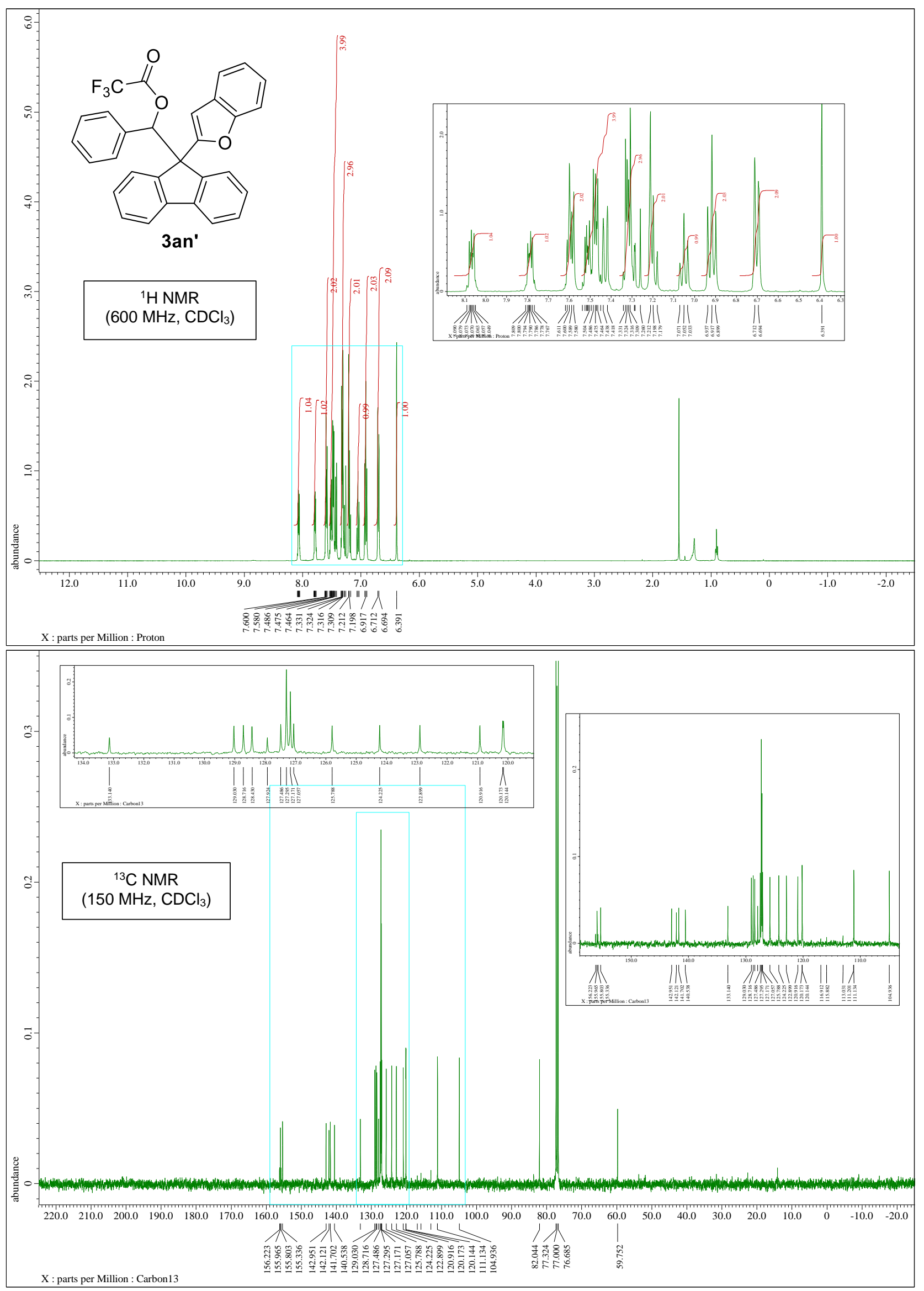




\section{Computational results}

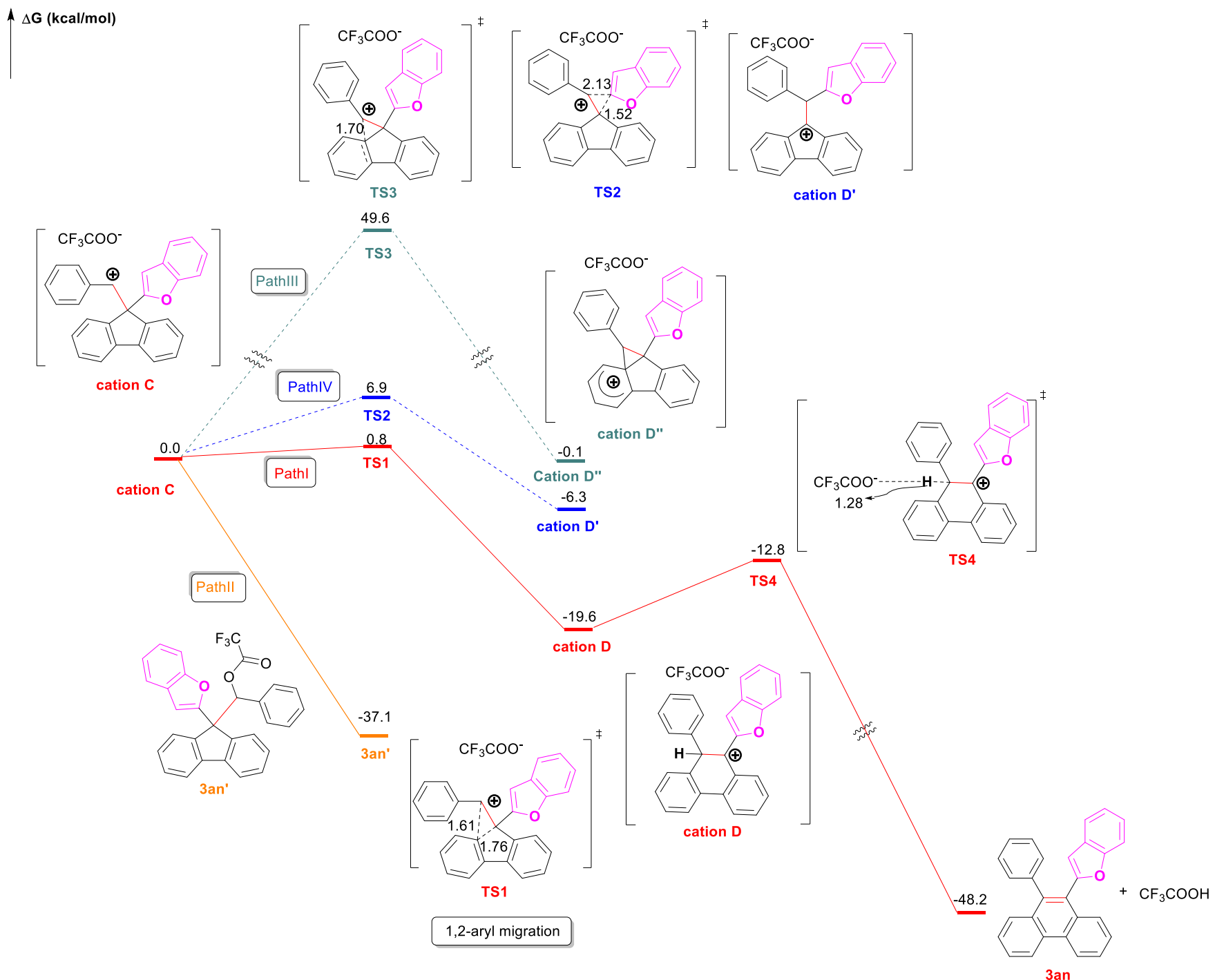

Path I: to the product; Path II: by product (intermediate to the product); Path III: high energy barrier, through cation D" is disfavored;

Path IV: relatively high energy barrier, cation $C$ is more likely through path I and II rather than through TS2 to cation D' (experimental result: 3 an was isolated, the other contepart TFA adduct did not detected).

Figure S2. Energy profile of oxidative ring expansion at the level of $\omega B 97 X D / 6-31 G(d, p)$, solvent $(2,2,2-$ trifluoroethanol). Bond lengths are shown in angstroms.

\section{Cation D'}

Sum of electronic and zero-point Energies=

$-1152.734632$

Sum of electronic and thermal Energies=

$-1152.713465$

Sum of electronic and thermal Enthalpies=

$-1152.712520$

Sum of electronic and thermal Free Energies=

$-1152.786661$

\section{Standard orientation:}

\begin{tabular}{cccccc} 
Center & Atomic & Atomic & & \multicolumn{2}{c}{ Coordinates (Angstroms) } \\
Number & Number & Type & X & Y & Z
\end{tabular}




\begin{tabular}{|c|c|c|c|c|c|}
\hline 2 & 6 & 0 & 0.376631 & -3.704654 & 0.560571 \\
\hline 3 & 6 & 0 & 0.422835 & -2.383408 & 0.117165 \\
\hline 4 & 6 & 0 & -0.784367 & -1.691465 & -0.045256 \\
\hline & 6 & 0 & -2.024058 & -2.341447 & 0.219492 \\
\hline 6 & 6 & 0 & -2.066440 & -3.637094 & 0.659000 \\
\hline 7 & 1 & 0 & -0.855220 & -5.344010 & 1.179283 \\
\hline 8 & 1 & 0 & 1.300004 & -4.256500 & 0.692423 \\
\hline & 1 & 0 & 1.379451 & -1.934339 & -0.111870 \\
\hline 10 & 1 & 0 & -3.006314 & -4.135222 & 0.870022 \\
\hline & 6 & 0 & -1.061190 & -0.346362 & -0.454995 \\
\hline 12 & 6 & 0 & -2.494508 & -0.166622 & -0.478073 \\
\hline 13 & 6 & 0 & -3.262714 & 0.938129 & -0.845878 \\
\hline 14 & 6 & 0 & -4.653419 & 0.818448 & -0.797213 \\
\hline 15 & 6 & 0 & -5.243820 & -0.370292 & -0.384826 \\
\hline 16 & 6 & 0 & -4.472694 & -1.491409 & -0.013229 \\
\hline 17 & 6 & 0 & -3.107921 & -1.374375 & -0.068064 \\
\hline 18 & 1 & 0 & -2.804200 & 1.868192 & -1.162222 \\
\hline 19 & 1 & 0 & -5.274832 & 1.660508 & -1.079638 \\
\hline 20 & 1 & 0 & -6.326132 & -0.441104 & -0.347327 \\
\hline 21 & 1 & 0 & -4.953774 & -2.411178 & 0.300987 \\
\hline 22 & 6 & 0 & -0.105889 & 0.738572 & -0.839099 \\
\hline 23 & 6 & 0 & -0.391175 & 2.039425 & -0.082582 \\
\hline 24 & 6 & 0 & -0.586499 & 2.034127 & 1.299876 \\
\hline 25 & 6 & 0 & -0.414567 & 3.245154 & -0.781967 \\
\hline 26 & 6 & 0 & -0.816651 & 3.229427 & 1.973245 \\
\hline 27 & 1 & 0 & -0.558428 & 1.099327 & 1.853214 \\
\hline 28 & 6 & 0 & -0.641596 & 4.439827 & -0.104485 \\
\hline 29 & 1 & 0 & -0.257741 & 3.248431 & -1.857033 \\
\hline 30 & 6 & 0 & -0.846331 & 4.433500 & 1.272959 \\
\hline 31 & 1 & 0 & -0.971539 & 3.219618 & 3.047559 \\
\hline 32 & 1 & 0 & -0.660804 & 5.374723 & -0.655636 \\
\hline 33 & 1 & 0 & -1.028553 & 5.364556 & 1.800468 \\
\hline 34 & 6 & 0 & 1.349544 & 0.405416 & -0.765666 \\
\hline 35 & 6 & 0 & 2.266453 & 0.167944 & -1.729142 \\
\hline 36 & 6 & 0 & 3.217946 & 0.046509 & 0.320082 \\
\hline 37 & 6 & 0 & 3.511902 & -0.078964 & -1.043082 \\
\hline 38 & 1 & 0 & 2.082427 & 0.170412 & -2.793826 \\
\hline 39 & 6 & 0 & 4.150772 & -0.122311 & 1.334042 \\
\hline 40 & 6 & 0 & 4.822143 & -0.394058 & -1.426491 \\
\hline 41 & 6 & 0 & 5.443295 & -0.430562 & 0.929713 \\
\hline 42 & 1 & 0 & 3.877967 & -0.019163 & 2.378366 \\
\hline 43 & 6 & 0 & 5.773438 & -0.564189 & -0.431099 \\
\hline 44 & 1 & 0 & 5.083239 & -0.501436 & -2.474413 \\
\hline 45 & 1 & 0 & 6.214508 & -0.573460 & 1.679907 \\
\hline 46 & 1 & 0 & 6.795594 & -0.807580 & -0.703527 \\
\hline 47 & 1 & 0 & -0.330838 & 0.918122 & -1.900627 \\
\hline 48 & 8 & 0 & 1.897461 & 0.345302 & 0.491187 \\
\hline
\end{tabular}


TS2

Sum of electronic and zero-point Energies=

$-1152.715879$

Sum of electronic and thermal Energies=

$-1152.695605$

Sum of electronic and thermal Enthalpies=

$-1152.694661$

Sum of electronic and thermal Free Energies=

$-1152.765591$

Standard orientation:

\begin{tabular}{cccccc} 
Center & Atomic & Atomic & & \multicolumn{3}{c}{ Coordinates (Angstroms) } \\
Number & Number & Type & X & Y & Z
\end{tabular}

$\begin{array}{cccccc}1 & 6 & 0 & 4.231944 & -2.018292 & -1.813270 \\ 2 & 6 & 0 & 3.097168 & -2.049095 & -2.623285 \\ 3 & 6 & 0 & 1.906132 & -1.453605 & -2.205485 \\ 4 & 6 & 0 & 1.871002 & -0.845657 & -0.957929 \\ 5 & 6 & 0 & 3.011145 & -0.805940 & -0.148636 \\ 6 & 6 & 0 & 4.199376 & -1.393437 & -0.568675 \\ 7 & 1 & 0 & 5.150013 & -2.484233 & -2.157095 \\ 8 & 1 & 0 & 3.137753 & -2.538100 & -3.591089 \\ 9 & 1 & 0 & 1.036363 & -1.482149 & -2.855582 \\ 10 & 1 & 0 & 5.084052 & -1.365060 & 0.059745 \\ 11 & 6 & 0 & 0.732068 & -0.084301 & -0.274259 \\ 12 & 6 & 0 & 1.350350 & 0.312486 & 1.066564 \\ 13 & 6 & 0 & 0.771256 & 0.882627 & 2.194652 \\ 14 & 6 & 0 & 1.561750 & 1.093738 & 3.324022 \\ 15 & 6 & 0 & 2.905159 & 0.719876 & 3.336496 \\ 16 & 6 & 0 & 3.475529 & 0.102968 & 2.226608 \\ 17 & 6 & 0 & 2.687320 & -0.108058 & 1.100152 \\ 18 & 1 & 0 & -0.276347 & 1.157611 & 2.213883 \\ 19 & 1 & 0 & 1.119272 & 1.549225 & 4.203996 \\ 20 & 1 & 0 & 3.504156 & 0.892955 & 4.224959 \\ 21 & 1 & 0 & 4.511071 & -0.221888 & 2.244251 \\ 22 & 6 & 0 & 0.094120 & 0.861711 & -1.232550 \\ 23 & 1 & 0 & 0.002034 & 0.423775 & -2.224223 \\ 24 & 6 & 0 & -0.507518 & 2.121275 & -1.101748 \\ 25 & 6 & 0 & -0.321807 & 3.003440 & -0.004127 \\ 26 & 6 & 0 & -1.323542 & 2.534740 & -2.192473 \\ 27 & 6 & 0 & -0.946982 & 4.231458 & 0.000516 \\ 28 & 1 & 0 & 0.341142 & 2.734768 & 0.805204 \\ 29 & 6 & 0 & -1.962817 & 3.756357 & -2.163814 \\ 30 & 1 & 0 & -1.447714 & 1.867624 & -3.039289 \\ 31 & 6 & 0 & -1.774215 & 4.600631 & -1.066928 \\ 32 & 1 & 0 & -0.794746 & 4.914348 & 0.828317 \\ 33 & 1 & 0 & -2.596618 & 4.063643 & -2.987583 \\ 34 & 1 & 0 & -2.267381 & 5.567330 & -1.047310\end{array}$




$\begin{array}{llllll}35 & 6 & 0 & -0.561163 & -0.866004 & -0.132700 \\ 36 & 6 & 0 & -1.055004 & -1.964219 & -0.771069 \\ 37 & 6 & 0 & -2.351873 & -2.197839 & -0.208622 \\ 38 & 1 & 0 & -0.555387 & -2.544749 & -1.532187 \\ 39 & 6 & 0 & -2.547711 & -1.175180 & 0.728987 \\ 40 & 6 & 0 & -3.361937 & -3.153567 & -0.399795 \\ 41 & 6 & 0 & -3.696845 & -1.038439 & 1.497038 \\ 42 & 6 & 0 & -4.517087 & -3.035259 & 0.352803 \\ 43 & 1 & 0 & -3.236925 & -3.957384 & -1.117464 \\ 44 & 6 & 0 & -4.681367 & -1.992704 & 1.287445 \\ 45 & 1 & 0 & -3.809645 & -0.233040 & 2.213580 \\ 46 & 1 & 0 & -5.315958 & -3.758587 & 0.226203 \\ 47 & 1 & 0 & -5.602505 & -1.935767 & 1.858415 \\ 48 & 8 & 0 & -1.461168 & -0.361074 & 0.771721\end{array}$

\section{Cation C}

Sum of electronic and zero-point Energies=

$-1152.724958$

Sum of electronic and thermal Energies $=$

$-1152.703744$

Sum of electronic and thermal Enthalpies=

Sum of electronic and thermal Free Energies=

$-1152.702800$

$-1152.776624$

Standard orientation:

\begin{tabular}{|c|c|c|c|c|c|}
\hline \multirow{2}{*}{$\begin{array}{l}\text { Center } \\
\text { Number }\end{array}$} & \multirow{2}{*}{\multicolumn{2}{|c|}{$\begin{array}{l}\text { Atomic } \\
\text { Number }\end{array}$}} & \multirow{2}{*}{$\begin{array}{l}\text { Atomic } \\
\text { Type }\end{array}$} & \multicolumn{2}{|c|}{ Coordinates (Angstroms } \\
\hline & & & & $x$ & Z \\
\hline 1 & 6 & 0 & -1.171635 & 4.273751 & 1.430627 \\
\hline 2 & 6 & 0 & -1.597287 & 3.196873 & 2.249393 \\
\hline 3 & 6 & 0 & -1.156100 & 1.885931 & 1.975046 \\
\hline 4 & 6 & 0 & -0.335517 & 1.710082 & 0.922007 \\
\hline 5 & 6 & 0 & 0.159822 & 2.776250 & 0.192585 \\
\hline 6 & 6 & 0 & -0.264106 & 4.039795 & 0.378244 \\
\hline 7 & 1 & 0 & -1.535468 & 5.263069 & 1.614477 \\
\hline 8 & 1 & 0 & -2.255971 & 3.378515 & 3.072833 \\
\hline 9 & 1 & 0 & -1.469166 & 1.058885 & 2.577404 \\
\hline 10 & 1 & 0 & 0.081481 & 4.838387 & -0.244405 \\
\hline 11 & 6 & 0 & 0.202045 & 0.389498 & 0.329911 \\
\hline 12 & 6 & 0 & 1.355266 & 0.881308 & -0.570977 \\
\hline 13 & 6 & 0 & 2.353166 & 0.165792 & -1.123645 \\
\hline 14 & 6 & 0 & 3.224917 & 0.833214 & -2.008528 \\
\hline 15 & 6 & 0 & 3.035868 & 2.211314 & -2.284646 \\
\hline 16 & 6 & 0 & 2.011138 & 2.924467 & -1.631037 \\
\hline 17 & 6 & 0 & 1.239838 & 2.246836 & -0.761146 \\
\hline 18 & 1 & 0 & 2.475292 & -0.874618 & -0.905622 \\
\hline 19 & 1 & 0 & 4.026532 & 0.299150 & -2.474380 \\
\hline 20 & 1 & 0 & 3.673162 & 2.709892 & -2.984771 \\
\hline
\end{tabular}




\begin{tabular}{llllll}
21 & 1 & 0 & 1.857646 & 3.967595 & -1.813266 \\
22 & 6 & 0 & 0.648482 & -0.625128 & 1.398878 \\
23 & 1 & 0 & 0.151691 & -0.657871 & 2.345983 \\
24 & 6 & 0 & 1.809890 & -1.592631 & 1.104739 \\
25 & 6 & 0 & 3.125871 & -1.221569 & 1.411999 \\
26 & 6 & 0 & 1.550771 & -2.844149 & 0.529828 \\
27 & 6 & 0 & 4.182762 & -2.102142 & 1.144410 \\
28 & 1 & 0 & 3.323653 & -0.265945 & 1.850896 \\
29 & 6 & 0 & 2.607578 & -3.724634 & 0.262227 \\
30 & 1 & 0 & 0.546020 & -3.127476 & 0.295191 \\
31 & 6 & 0 & 3.923570 & -3.353697 & 0.569517 \\
32 & 1 & 0 & 5.187575 & -1.818897 & 1.379030 \\
33 & 1 & 0 & 2.409683 & -4.680169 & -0.176725 \\
34 & 1 & 0 & 4.730401 & -4.026095 & 0.365172 \\
35 & 6 & 0 & -0.886488 & -0.270258 & -0.536930 \\
36 & 6 & 0 & -2.069481 & 0.419975 & -1.177010 \\
37 & 6 & 0 & -3.033653 & -0.444849 & -0.866252 \\
38 & 1 & 0 & -2.116891 & 1.322343 & -1.750062 \\
39 & 6 & 0 & -2.328823 & -1.774927 & -0.600491 \\
40 & 6 & 0 & -4.547368 & -0.328762 & -0.790089 \\
41 & 6 & 0 & -3.170787 & -2.990283 & -0.229776 \\
42 & 6 & 0 & -5.248580 & -1.458393 & -0.449664 \\
43 & 1 & 0 & -5.045827 & 0.596054 & -0.992893 \\
44 & 6 & 0 & -4.535987 & -2.832941 & -0.156857 \\
45 & 1 & 0 & -2.714072 & -3.937719 & -0.033081 \\
46 & 1 & 0 & -6.314984 & -1.403925 & -0.381030 \\
47 & 1 & 0 & -5.137278 & -3.677478 & 0.107965 \\
48 & 8 & 0 & -1.048064 & -1.663503 & -0.765862 \\
& & & & & \\
\hline & & & &
\end{tabular}

TS1

Sum of electronic and zero-point Energies=

$-1152.724993$

$-1152.704458$

Sum of electronic and thermal Energies=

Sum of electronic and thermal Enthalpies=

Sum of electronic and thermal Free Energies=

$-1152.703513$

$-1152.775287$

Standard orientation:

\begin{tabular}{|c|c|c|c|c|}
\hline Center & Atomic & Atomic & Coordinate & (Angstroms \\
\hline Number & Number & r Type & $x$ & Z \\
\hline 1 & 6 & -1.135721 & 3.521501 & 1.791800 \\
\hline 2 & 6 & -0.218594 & 3.687747 & 0.745541 \\
\hline 3 & 6 & 0.109005 & 2.616336 & -0.060204 \\
\hline 4 & 6 & -0.525251 & 1.373278 & 0.165933 \\
\hline 5 & 6 & -1.374511 & 1.188094 & 1.296336 \\
\hline
\end{tabular}




\begin{tabular}{cccccc}
6 & 6 & 0 & -1.698746 & 2.279697 & 2.083943 \\
7 & 1 & 0 & -1.383184 & 4.372708 & 2.417945 \\
8 & 1 & 0 & 0.228157 & 4.658669 & 0.565667 \\
9 & 1 & 0 & 0.793837 & 2.729805 & -0.893762 \\
10 & 1 & 0 & -2.335277 & 2.156362 & 2.953448 \\
11 & 6 & 0 & 0.097783 & -0.250776 & -0.083849 \\
12 & 6 & 0 & -0.737058 & -1.036886 & 0.883843 \\
13 & 6 & 0 & -0.757739 & -2.413352 & 1.016013 \\
14 & 6 & 0 & -1.684887 & -2.975042 & 1.899280 \\
15 & 6 & 0 & -2.574249 & -2.172052 & 2.609427 \\
16 & 6 & 0 & -2.551672 & -0.784656 & 2.465403 \\
17 & 6 & 0 & -1.626234 & -0.224469 & 1.594999 \\
18 & 1 & 0 & -0.071926 & -3.038715 & 0.454620 \\
19 & 1 & 0 & -1.708021 & -4.051551 & 2.032343 \\
20 & 1 & 0 & -3.287835 & -2.629001 & 3.286825 \\
21 & 1 & 0 & -3.238573 & -0.156240 & 3.023027 \\
22 & 6 & 0 & -0.637013 & 0.421476 & -1.123941 \\
23 & 1 & 0 & -0.027996 & 0.857648 & -1.912816 \\
24 & 6 & 0 & -2.013845 & 0.016728 & -1.554569 \\
25 & 6 & 0 & -3.045886 & 0.952515 & -1.614339 \\
26 & 6 & 0 & -2.241226 & -1.298282 & -1.962315 \\
27 & 6 & 0 & -4.303076 & 0.571724 & -2.072017 \\
28 & 1 & 0 & -2.868269 & 1.974880 & -1.292520 \\
29 & 6 & 0 & -3.499599 & -1.675099 & -2.422157 \\
30 & 1 & 0 & -1.435525 & -2.025034 & -1.918595 \\
31 & 6 & 0 & -4.532057 & -0.742275 & -2.475277 \\
32 & 1 & 0 & -5.105388 & 1.301732 & -2.111107 \\
33 & 1 & 0 & -3.672725 & -2.700285 & -2.733855 \\
34 & 1 & 0 & -5.514702 & -1.038904 & -2.828554 \\
35 & 6 & 0 & 1.537207 & -0.373317 & -0.071968 \\
36 & 6 & 0 & 2.366701 & -1.000555 & 0.804296 \\
37 & 6 & 0 & 3.696878 & -0.764839 & 0.321235 \\
38 & 1 & 0 & 2.065749 & -1.558048 & 1.679540 \\
39 & 6 & 0 & 3.546219 & 0.018253 & -0.831289 \\
40 & 6 & 0 & 4.986889 & -1.122995 & 0.740235 \\
41 & 6 & 0 & 4.607498 & 0.472138 & -1.602340 \\
42 & 6 & 0 & 6.060880 & -0.679592 & -0.012778 \\
43 & 1 & 0 & 5.134107 & -1.727526 & 1.628969 \\
44 & 6 & 0 & 5.873223 & 0.106137 & -1.166857 \\
45 & 1 & 0 & 4.449326 & 1.075365 & -2.488902 \\
& 1 & 0 & 7.070494 & -0.939812 & 0.287982 \\
47 & 0 & 6.741056 & 0.433686 & -1.730232 \\
\hline & 0 & 2.227148 & 0.254454 & -1.075793 \\
\hline & & & & & \\
\hline
\end{tabular}

\section{Cation D}


Standard orientation:

\begin{tabular}{|c|c|c|c|c|c|}
\hline \multirow{2}{*}{$\begin{array}{l}\text { Center } \\
\text { Number }\end{array}$} & \multicolumn{2}{|l|}{ Atomic } & Atomic & \multicolumn{2}{|c|}{ Coordinates (Angstroms } \\
\hline & Number & & Type & $x$ & Z \\
\hline 1 & 6 & 0 & -1.762048 & 3.698773 & -1.539777 \\
\hline 2 & 6 & 0 & -1.072205 & 3.047755 & -2.574317 \\
\hline 3 & 6 & 0 & -0.585343 & 1.748165 & -2.380156 \\
\hline 4 & 6 & 0 & -0.781432 & 1.133728 & -1.190789 \\
\hline 5 & 6 & 0 & -1.494995 & 1.793494 & -0.124067 \\
\hline 6 & 6 & 0 & -1.968361 & 3.049309 & -0.312707 \\
\hline 7 & 1 & 0 & -2.132068 & 4.691888 & -1.687052 \\
\hline 8 & 1 & 0 & -0.918348 & 3.543122 & -3.510166 \\
\hline 9 & 1 & 0 & -0.061803 & 1.245701 & -3.166540 \\
\hline 10 & 1 & 0 & -2.499439 & 3.545022 & 0.472880 \\
\hline 11 & 6 & 0 & 0.132380 & -0.229428 & 0.520642 \\
\hline 12 & 6 & 0 & -1.019625 & -0.114228 & 1.477366 \\
\hline 13 & 6 & 0 & -1.336507 & -0.988837 & 2.453205 \\
\hline 14 & 6 & 0 & -2.336743 & -0.624410 & 3.367212 \\
\hline 15 & 6 & 0 & -2.972039 & 0.627715 & 3.238458 \\
\hline 16 & 6 & 0 & -2.662764 & 1.462937 & 2.148384 \\
\hline 17 & 6 & 0 & -1.752526 & 1.042572 & 1.240217 \\
\hline 18 & 1 & 0 & -0.830958 & -1.928244 & 2.536130 \\
\hline 19 & 1 & 0 & -2.610907 & -1.289083 & 4.159632 \\
\hline 20 & 1 & 0 & -3.693034 & 0.939863 & 3.964855 \\
\hline 21 & 1 & 0 & -3.149256 & 2.408988 & 2.033119 \\
\hline 22 & 6 & 0 & -0.273632 & -0.297321 & -0.918033 \\
\hline 23 & 1 & 0 & 0.553837 & -0.577144 & -1.536011 \\
\hline 24 & 6 & 0 & -1.415579 & -1.304417 & -1.148964 \\
\hline 25 & 6 & 0 & -2.748574 & -0.898483 & -0.999907 \\
\hline 26 & 6 & 0 & -1.121820 & -2.626749 & -1.508146 \\
\hline 27 & 6 & 0 & -3.787816 & -1.814872 & -1.210030 \\
\hline 28 & 1 & 0 & -2.972858 & 0.111156 & -0.725657 \\
\hline 29 & 6 & 0 & -2.161067 & -3.543147 & -1.718326 \\
\hline 30 & 1 & 0 & -0.104047 & -2.936688 & -1.621911 \\
\hline 31 & 6 & 0 & -3.494067 & -3.137203 & -1.569278 \\
\hline 32 & 1 & 0 & -4.805583 & -1.504917 & -1.096169 \\
\hline 33 & 1 & 0 & -1.936778 & -4.552779 & -1.992602 \\
\hline 34 & 1 & 0 & -4.287551 & -3.836888 & -1.729773 \\
\hline 35 & 6 & 0 & 1.603647 & -0.170505 & 0.971772 \\
\hline 36 & 6 & 0 & 2.469089 & 1.065872 & 1.061973 \\
\hline 37 & 6 & 0 & 3.579955 & 0.609422 & 0.486054 \\
\hline
\end{tabular}




$\begin{array}{llllll}38 & 1 & 0 & 2.266294 & 2.021740 & 1.497984 \\ 39 & 6 & 0 & 3.519833 & -0.912418 & 0.615409 \\ 40 & 6 & 0 & 4.771588 & 1.296562 & -0.160832 \\ 41 & 6 & 0 & 4.671101 & -1.742128 & 0.059012 \\ 42 & 6 & 0 & 5.774730 & 0.493995 & -0.643744 \\ 43 & 1 & 0 & 4.825774 & 2.362432 & -0.237238 \\ 44 & 6 & 0 & 5.720398 & -1.076846 & -0.532787 \\ 45 & 1 & 0 & 4.664074 & -2.809913 & 0.127389 \\ 46 & 1 & 0 & 6.622245 & 0.950331 & -1.111042 \\ 47 & 1 & 0 & 6.531756 & -1.648690 & -0.932255 \\ 48 & 8 & 0 & 2.445648 & -1.283969 & 1.238420\end{array}$

TS4

Sum of electronic and zero-point Energies=

$-1678.926809$

$-1678.899537$

Sum of electronic and thermal Energies=

$-1678.898593$

Sum of electronic and thermal Enthalpies

$-1678.987654$

Standard orientation:

\begin{tabular}{|c|c|c|c|c|c|}
\hline Center & Atomic & & omic & Coordinates & (Angstroms \\
\hline Number & Numbe & & Type & $x$ & z \\
\hline 1 & 6 & 0 & -4.692182 & -2.283102 & -0.844858 \\
\hline 2 & 6 & 0 & -3.684993 & -3.033067 & -0.226432 \\
\hline 3 & 6 & 0 & -2.550360 & -2.405257 & 0.247000 \\
\hline 4 & 6 & 0 & -2.389948 & -1.014022 & 0.122174 \\
\hline 5 & 6 & 0 & -3.413993 & -0.250665 & -0.473882 \\
\hline 6 & 6 & 0 & -4.557042 & -0.915580 & -0.962463 \\
\hline 7 & 1 & 0 & -5.580984 & -2.772277 & -1.229704 \\
\hline 8 & 1 & 0 & -3.789790 & -4.108286 & -0.122769 \\
\hline 9 & 1 & 0 & -1.769252 & -2.994233 & 0.712809 \\
\hline 10 & 1 & 0 & -5.353020 & -0.359711 & -1.442746 \\
\hline 11 & 6 & U & -0.991925 & 1.044563 & 0.392457 \\
\hline 12 & 6 & U & -2.107954 & 1.840333 & -0.032010 \\
\hline 13 & 6 & 0 & -2.071548 & 3.254720 & 0.076806 \\
\hline 14 & 6 & 0 & -3.132051 & 4.025659 & -0.335071 \\
\hline 15 & 6 & 0 & -4.266405 & 3.402539 & -0.872577 \\
\hline 16 & 6 & 0 & -4.347204 & 2.026763 & -0.939747 \\
\hline 17 & 6 & 0 & -3.293292 & 1.205494 & -0.499462 \\
\hline 18 & 1 & 0 & -1.217783 & 3.735964 & 0.535802 \\
\hline 19 & 1 & 0 & -3.094328 & 5.104350 & -0.230174 \\
\hline 20 & 1 & 0 & -5.105302 & 4.002841 & -1.210145 \\
\hline 21 & 1 & 0 & -5.260019 & 1.587263 & -1.320474 \\
\hline 22 & 6 & 0 & -1.110233 & -0.383553 & 0.520286 \\
\hline
\end{tabular}




\begin{tabular}{rrrrrr}
23 & 1 & 0 & -0.361528 & -0.572654 & -0.502392 \\
24 & 6 & 0 & -0.384233 & -1.038037 & 1.683993 \\
25 & 6 & 0 & -0.650540 & -0.559854 & 2.970945 \\
26 & 6 & 0 & 0.504176 & -2.102294 & 1.526488 \\
27 & 6 & 0 & -0.014317 & -1.113988 & 4.076713 \\
28 & 1 & 0 & -1.352615 & 0.258488 & 3.104375 \\
29 & 6 & 0 & 1.139452 & -2.656674 & 2.634342 \\
30 & 1 & 0 & 0.692134 & -2.516761 & 0.543122 \\
31 & 6 & 0 & 0.889922 & -2.160022 & 3.910625 \\
32 & 1 & 0 & -0.226039 & -0.727138 & 5.068899 \\
33 & 1 & 0 & 1.833400 & -3.479928 & 2.494916 \\
34 & 1 & 0 & 1.391849 & -2.589751 & 4.772030 \\
35 & 6 & 0 & 0.324453 & 1.636863 & 0.459998 \\
36 & 6 & 0 & 0.877313 & 2.611947 & -0.332341 \\
37 & 6 & 0 & 2.289148 & 2.539449 & -0.141236 \\
38 & 1 & 0 & 0.354715 & 3.246690 & -1.031966 \\
39 & 6 & 0 & 2.487111 & 1.506882 & 0.783130 \\
40 & 6 & 0 & 3.401836 & 3.231762 & -0.648651 \\
41 & 6 & 0 & 3.735465 & 1.121840 & 1.256902 \\
42 & 6 & 0 & 4.654215 & 2.860384 & -0.196439 \\
43 & 1 & 0 & 3.275769 & 4.032196 & -1.370010 \\
44 & 6 & 0 & 4.817009 & 1.819758 & 0.743509 \\
45 & 1 & 0 & 3.847400 & 0.319747 & 1.977424 \\
46 & 1 & 0 & 5.534108 & 3.375909 & -0.567138 \\
47 & 1 & 0 & 5.817849 & 1.558890 & 1.072516 \\
48 & 8 & 0 & 1.303355 & 0.963583 & 1.146081 \\
49 & 8 & 0 & 0.702825 & -0.597583 & -1.420176 \\
50 & 6 & 0 & 1.033193 & -1.766735 & -1.792170 \\
51 & 8 & 0 & 0.356489 & -2.793631 & -1.807209 \\
52 & 6 & 0 & 2.534556 & -1.867147 & -2.149440 \\
53 & 9 & 0 & 2.961092 & -0.808188 & -2.849937 \\
54 & 9 & 0 & 2.820100 & -2.968922 & -2.849253 \\
55 & 9 & 0 & 3.250399 & -1.906702 & -1.006974 \\
------------------------------------------------------- \\
\hline
\end{tabular}

3an

Sum of electronic and zero-point Energies $=$

$-1678.980871$

Sum of electronic and thermal Energies=

$-1678.952115$

Sum of electronic and thermal Enthalpies=

$-1678.951171$

Sum of electronic and thermal Free Energies=

$-1679.043994$

Standard orientation:

\begin{tabular}{lcccc} 
Center & Atomic Atomic & & \multicolumn{2}{c}{ Coordinates (Angstroms) } \\
Number Number & Type & & X & Y Z
\end{tabular}




\begin{tabular}{|c|c|c|c|c|c|}
\hline 1 & 6 & 0 & 3.602036 & 3.626536 & 0.362007 \\
\hline 2 & 6 & 0 & 2.648152 & 3.968717 & -0.607936 \\
\hline 3 & 6 & 0 & 1.487295 & 3.236386 & -0.709244 \\
\hline 4 & 6 & 0 & 1.236221 & 2.145741 & 0.154453 \\
\hline 5 & 6 & 0 & 2.204849 & 1.788413 & 1.125643 \\
\hline 6 & 6 & 0 & 3.385869 & 2.557348 & 1.204056 \\
\hline 7 & 1 & 0 & 4.519479 & 4.200343 & 0.447424 \\
\hline 8 & 1 & 0 & 2.825603 & 4.803698 & -1.277783 \\
\hline 9 & 1 & 0 & 0.753596 & 3.495629 & -1.463765 \\
\hline 10 & 1 & 0 & 4.150152 & 2.311725 & 1.931397 \\
\hline 11 & 6 & 0 & -0.205742 & 0.293790 & 0.854170 \\
\hline 12 & 6 & 0 & 0.772433 & -0.102135 & 1.858360 \\
\hline 13 & 6 & 0 & 0.546324 & -1.211225 & 2.701673 \\
\hline 14 & 6 & 0 & 1.476639 & -1.584282 & 3.645200 \\
\hline 15 & 6 & 0 & 2.668258 & -0.856137 & 3.776853 \\
\hline 16 & 6 & 0 & 2.905684 & 0.232253 & 2.966756 \\
\hline 17 & 6 & 0 & 1.971616 & 0.637893 & 1.988179 \\
\hline 18 & 1 & 0 & -0.375601 & -1.772723 & 2.605926 \\
\hline 19 & 1 & 0 & 1.286038 & -2.440111 & 4.284791 \\
\hline 20 & 1 & 0 & 3.404354 & -1.147376 & 4.519307 \\
\hline 21 & 1 & 0 & 3.830614 & 0.780513 & 3.096523 \\
\hline 22 & 6 & 0 & 0.006988 & 1.390542 & 0.049089 \\
\hline 23 & 1 & 0 & 0.718848 & -0.673488 & -0.582433 \\
\hline 24 & 6 & 0 & -1.044315 & 1.806487 & -0.926445 \\
\hline 25 & 6 & 0 & -1.778569 & 2.974266 & -0.695475 \\
\hline 26 & 6 & 0 & -1.339815 & 1.023105 & -2.044296 \\
\hline 27 & 6 & 0 & -2.798492 & 3.346433 & -1.564910 \\
\hline 28 & 1 & 0 & -1.553007 & 3.584710 & 0.173982 \\
\hline 29 & 6 & 0 & -2.359611 & 1.398769 & -2.914040 \\
\hline 30 & 1 & 0 & -0.770041 & 0.118267 & -2.233165 \\
\hline 31 & 6 & 0 & -3.093138 & 2.557730 & -2.674846 \\
\hline 32 & 1 & 0 & -3.365507 & 4.251815 & -1.371797 \\
\hline 33 & 1 & 0 & -2.581661 & 0.782833 & -3.779911 \\
\hline 34 & 1 & 0 & -3.891439 & 2.846594 & -3.351467 \\
\hline 35 & 6 & 0 & -1.507384 & -0.407150 & 0.779855 \\
\hline 36 & 6 & 0 & -1.858796 & -1.656729 & 0.395917 \\
\hline 37 & 6 & 0 & -3.297562 & -1.711259 & 0.465727 \\
\hline 38 & 1 & 0 & -1.181720 & -2.435616 & 0.076474 \\
\hline 39 & 6 & 0 & -3.697088 & -0.444062 & 0.905947 \\
\hline 40 & 6 & 0 & -4.277342 & -2.676830 & 0.202883 \\
\hline 41 & 6 & 0 & -5.019672 & -0.074959 & 1.105856 \\
\hline 42 & 6 & 0 & -5.607850 & -2.330398 & 0.392949 \\
\hline 43 & 1 & 0 & -4.002774 & -3.668834 & -0.141154 \\
\hline 44 & 6 & 0 & -5.974523 & -1.048184 & 0.838258 \\
\hline 45 & 1 & 0 & -5.284748 & 0.918991 & 1.447527 \\
\hline 46 & 1 & 0 & -6.384282 & -3.062705 & 0.194627 \\
\hline 47 & 1 & 0 & -7.025186 & -0.812382 & 0.974760 \\
\hline
\end{tabular}




$\begin{array}{llllll}48 & 8 & 0 & -2.607834 & 0.350664 & 1.105999 \\ 49 & 8 & 0 & 1.078377 & -1.568592 & -1.218837 \\ 50 & 6 & 0 & 2.382611 & -1.523021 & -1.268114 \\ 51 & 8 & 0 & 3.117345 & -0.685991 & -0.799433 \\ 52 & 6 & 0 & 2.942231 & -2.751664 & -2.015146 \\ 53 & 9 & 0 & 2.734487 & -3.860290 & -1.292417 \\ 54 & 9 & 0 & 4.250928 & -2.617756 & -2.216348 \\ 55 & 9 & 0 & 2.343512 & -2.909596 & -3.199389\end{array}$

3an'

Sum of electronic and zero-point Energies=

$-1678.966498$

Sum of electronic and thermal Energies=

$-1678.939085$

Sum of electronic and thermal Enthalpies=

$-1678.938141$

Sum of electronic and thermal Free Energies=

$-1679.026289$

Standard orientation:

\begin{tabular}{|c|c|c|c|c|c|}
\hline \multirow{2}{*}{$\begin{array}{l}\text { Center } \\
\text { Number }\end{array}$} & \multirow{2}{*}{\multicolumn{2}{|c|}{$\begin{array}{l}\text { Atomic } \\
\text { Number }\end{array}$}} & \multirow{2}{*}{$\begin{array}{l}\text { Atomic } \\
\text { Type }\end{array}$} & \multicolumn{2}{|c|}{ Coordinates (Angstroms } \\
\hline & & & & $x$ & Z \\
\hline 1 & 6 & 0 & -3.370693 & -2.116848 & 3.109354 \\
\hline 2 & 6 & 0 & -2.219178 & -1.469024 & 3.559219 \\
\hline 3 & 6 & 0 & -1.286074 & -0.967813 & 2.651876 \\
\hline 4 & 6 & 0 & -1.537976 & -1.140095 & 1.298137 \\
\hline 5 & 6 & 0 & -2.692550 & -1.789140 & 0.836107 \\
\hline 6 & 6 & 0 & -3.616241 & -2.287172 & 1.747221 \\
\hline 7 & 1 & 0 & -4.084205 & -2.499969 & 3.831992 \\
\hline 8 & 1 & 0 & -2.045649 & -1.355071 & 4.624109 \\
\hline 9 & 1 & 0 & -0.385560 & -0.469608 & 2.996300 \\
\hline 10 & 1 & & -4.508508 & -2.802367 & 1.405940 \\
\hline 11 & 6 & U & -0.689677 & -0.680939 & 0.097612 \\
\hline 12 & 6 & 0 & -1.498643 & -1.205778 & -1.081424 \\
\hline 13 & 6 & 0 & -1.204183 & -1.110333 & -2.432363 \\
\hline 14 & 6 & 0 & -2.110941 & -1.654689 & -3.341991 \\
\hline 15 & 6 & 0 & -3.280724 & -2.276138 & -2.899149 \\
\hline 16 & 6 & & -3.567524 & -2.377386 & -1.539200 \\
\hline 17 & 6 & 0 & -2.662557 & -1.840441 & -0.629333 \\
\hline 18 & 1 & 0 & -0.299310 & -0.618370 & -2.773790 \\
\hline 19 & 1 & 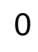 & -1.904642 & -1.593821 & -4.405720 \\
\hline 20 & 1 & 0 & -3.974157 & -2.689452 & -3.624700 \\
\hline 21 & 1 & 0 & -4.475836 & -2.864284 & -1.198338 \\
\hline 22 & 6 & 0 & -0.649967 & 0.796439 & 0.306044 \\
\hline 23 & 1 & 0 & -0.623281 & 0.908308 & 1.369845 \\
\hline 24 & 6 & 0 & -1.479918 & 1.827090 & -0.121393 \\
\hline 25 & 6 & 0 & -2.618006 & 1.682831 & -0.973431 \\
\hline
\end{tabular}




\begin{tabular}{lrrrrr}
26 & 6 & 0 & -1.147886 & 3.126602 & 0.373881 \\
27 & 6 & 0 & -3.373847 & 2.786234 & -1.291483 \\
28 & 1 & 0 & -2.894291 & 0.714046 & -1.364587 \\
29 & 6 & 0 & -1.909252 & 4.222954 & 0.034986 \\
30 & 1 & 0 & -0.284198 & 3.235114 & 1.021818 \\
31 & 6 & 0 & -3.020217 & 4.048940 & -0.794077 \\
32 & 1 & 0 & -4.241620 & 2.683339 & -1.932371 \\
33 & 1 & 0 & -1.653839 & 5.207482 & 0.408372 \\
34 & 1 & 0 & -3.624120 & 4.910502 & -1.061328 \\
35 & 6 & 0 & 0.701307 & -1.239444 & 0.122960 \\
36 & 6 & 0 & 1.292857 & -2.226342 & 0.828837 \\
37 & 6 & 0 & 2.648441 & -2.303195 & 0.337671 \\
38 & 1 & 0 & 0.833363 & -2.825690 & 1.600895 \\
39 & 6 & 0 & 2.749635 & -1.316391 & -0.649363 \\
40 & 6 & 0 & 3.771273 & -3.087961 & 0.626952 \\
41 & 6 & 0 & 3.904415 & -1.056265 & -1.372413 \\
42 & 6 & 0 & 4.939045 & -2.848644 & -0.083967 \\
43 & 1 & 0 & 3.727529 & -3.862338 & 1.386019 \\
44 & 6 & 0 & 5.005534 & -1.847740 & -1.068913 \\
45 & 1 & 0 & 3.938410 & -0.280499 & -2.129155 \\
46 & 1 & 0 & 5.822190 & -3.445005 & 0.122564 \\
47 & 1 & 0 & 5.936148 & -1.689488 & -1.604452 \\
48 & 8 & 0 & 1.557520 & -0.662452 & -0.782926 \\
49 & 8 & 0 & 0.646766 & 1.178421 & -0.160295 \\
50 & 6 & 0 & 1.241407 & 2.022634 & 0.720112 \\
51 & 8 & 0 & 0.615119 & 2.321507 & 1.735986 \\
52 & 6 & 0 & 2.643053 & 2.459390 & 0.255103 \\
53 & 9 & 0 & 2.832645 & 3.761040 & 0.558847 \\
54 & 9 & 0 & 3.577598 & 1.711003 & 0.878835 \\
55 & 9 & 0 & 2.747630 & 2.288996 & -1.080011 \\
\hline------------------------------------------------------- \\
\hline 3
\end{tabular}

TS3

Sum of electronic and zero-point Energies=

$-1152.646466$

Sum of electronic and thermal Energies=

$-1152.625741$

Sum of electronic and thermal Enthalpies=

$-1152.624797$

Sum of electronic and thermal Free Energies=

$-1152.697547$

Standard orientation:

\begin{tabular}{cccccc} 
Center & \multicolumn{2}{c}{ Atomic } & \multicolumn{2}{c}{ Atomic } & \multicolumn{2}{c}{ Coordinates } & (Angstroms) \\
Number & Number & Type & X & $Y$ & Z \\
- & & & & & \\
1 & 6 & 0 & -2.779833 & -2.251673 & -2.065024 \\
2 & 6 & 0 & -1.685927 & -2.924433 & -1.486991 \\
3 & 6 & 0 & -0.626001 & -2.245603 & -0.913432
\end{tabular}




\begin{tabular}{|c|c|c|c|c|c|}
\hline 4 & 6 & 0 & -0.671252 & -0.843746 & -0.885316 \\
\hline 5 & 6 & 0 & -1.737298 & -0.165284 & -1.545975 \\
\hline 6 & 6 & 0 & -2.812074 & -0.872207 & -2.099350 \\
\hline 7 & 1 & 0 & -3.595037 & -2.825806 & -2.491358 \\
\hline 8 & 1 & 0 & -1.666690 & -4.009650 & -1.504040 \\
\hline 9 & 1 & 0 & 0.218756 & -2.777451 & -0.489219 \\
\hline 10 & 1 & 0 & -3.646870 & -0.337634 & -2.540312 \\
\hline 11 & 6 & 0 & 0.322335 & 0.050279 & -0.397209 \\
\hline 12 & 6 & 0 & -0.428699 & 1.357657 & -0.398268 \\
\hline 13 & 6 & 0 & -0.018812 & 2.629516 & 0.110195 \\
\hline 14 & 6 & 0 & -0.760824 & 3.718524 & -0.255341 \\
\hline 15 & 6 & 0 & -1.875101 & 3.581706 & -1.125865 \\
\hline 16 & 6 & 0 & -2.288602 & 2.364835 & -1.636916 \\
\hline 17 & 6 & 0 & -1.560514 & 1.226248 & -1.289118 \\
\hline 18 & 1 & 0 & 0.849051 & 2.706629 & 0.755229 \\
\hline 19 & 1 & 0 & -0.495959 & 4.704357 & 0.108879 \\
\hline 20 & 1 & 0 & -2.428116 & 4.476619 & -1.393567 \\
\hline 21 & 1 & 0 & -3.152375 & 2.295933 & -2.287876 \\
\hline 22 & 6 & 0 & -0.569728 & 0.104842 & 0.734162 \\
\hline 23 & 1 & 0 & 0.010701 & 0.304020 & 1.651560 \\
\hline 24 & 6 & 0 & -1.952111 & -0.273804 & 1.224389 \\
\hline 25 & 6 & 0 & -2.938918 & 0.697808 & 1.381985 \\
\hline 26 & 6 & 0 & -2.163274 & -1.567454 & 1.701027 \\
\hline 27 & 6 & 0 & -4.159928 & 0.356183 & 1.954342 \\
\hline 28 & 1 & 0 & -2.758044 & 1.719603 & 1.067517 \\
\hline 29 & 6 & 0 & -3.385852 & -1.899515 & 2.276708 \\
\hline 30 & 1 & 0 & -1.381955 & -2.315229 & 1.624073 \\
\hline 31 & 6 & 0 & -4.390707 & -0.943869 & 2.394650 \\
\hline 32 & 1 & 0 & -4.928636 & 1.114876 & 2.060096 \\
\hline 33 & 1 & 0 & -3.548021 & -2.911869 & 2.632598 \\
\hline 34 & 1 & 0 & -5.344943 & -1.207938 & 2.839075 \\
\hline 35 & 6 & 0 & 1.763723 & -0.154971 & -0.280990 \\
\hline 36 & 6 & 0 & 2.620546 & -0.865506 & -1.045801 \\
\hline 37 & 6 & 0 & 3.921098 & -0.666729 & -0.454535 \\
\hline 38 & 1 & 0 & 2.363081 & -1.454056 & -1.914379 \\
\hline 39 & 6 & 0 & 3.712646 & 0.176541 & 0.642985 \\
\hline 40 & 6 & 0 & 5.221463 & -1.104002 & -0.736448 \\
\hline 41 & 6 & 0 & 4.722069 & 0.618288 & 1.485752 \\
\hline 42 & 6 & 0 & 6.248513 & -0.676018 & 0.091657 \\
\hline 43 & 1 & 0 & 5.417322 & -1.757608 & -1.580107 \\
\hline 44 & 6 & 0 & 6.002912 & 0.172165 & 1.186462 \\
\hline 45 & 1 & 0 & 4.515963 & 1.272132 & 2.325551 \\
\hline 46 & 1 & 0 & 7.265254 & -1.000046 & -0.106324 \\
\hline 47 & 1 & 0 & 6.832499 & 0.485674 & 1.812000 \\
\hline 48 & 8 & 0 & 2.386865 & 0.489847 & 0.753023 \\
\hline
\end{tabular}




\section{Cation D"}

Sum of electronic and zero-point Energies=

$-1152.725015$

Sum of electronic and thermal Energies=

$-1152.703863$

Sum of electronic and thermal Enthalpies=

$-1152.702919$

Sum of electronic and thermal Free Energies=

$-1152.776735$

Standard orientation:

\begin{tabular}{|c|c|c|c|c|c|}
\hline \multirow{2}{*}{$\begin{array}{l}\text { Center } \\
\text { Number }\end{array}$} & \multirow{2}{*}{\multicolumn{2}{|c|}{$\begin{array}{l}\text { Atomic } \\
\text { Number }\end{array}$}} & \multirow{2}{*}{$\begin{array}{l}\text { Atomic } \\
\text { Type }\end{array}$} & \multicolumn{2}{|c|}{ Coordinates (Angstroms) } \\
\hline & & & & $\begin{array}{ll}X & Y\end{array}$ & Z \\
\hline 1 & 6 & 0 & 2.336810 & -1.482819 & 3.183933 \\
\hline 2 & 6 & 0 & 1.340752 & -2.367290 & 2.686069 \\
\hline 3 & 6 & 0 & 0.484676 & -1.965721 & 1.638245 \\
\hline 4 & 6 & 0 & 0.651345 & -0.684366 & 1.126240 \\
\hline 5 & 6 & 0 & 1.664750 & 0.165174 & 1.573272 \\
\hline 6 & 6 & 0 & 2.505585 & -0.198958 & 2.619802 \\
\hline 7 & 1 & 0 & 2.965889 & -1.793694 & 3.991684 \\
\hline 8 & 1 & 0 & 1.238396 & -3.344284 & 3.110234 \\
\hline 9 & 1 & 0 & -0.268038 & -2.622312 & 1.254778 \\
\hline 10 & 1 & 0 & 3.258043 & $3 \quad 0.469647$ & 2.982462 \\
\hline 11 & 6 & 0 & -0.137105 & 0.045556 & 0.030689 \\
\hline 12 & 6 & 0 & 0.619044 & 1.366308 & -0.179483 \\
\hline 13 & 6 & 0 & 0.066351 & 2.635329 & -0.428525 \\
\hline 14 & 6 & 0 & 0.899299 & 3.773397 & -0.456084 \\
\hline 15 & 6 & 0 & 2.190472 & 3.706317 & 0.097117 \\
\hline 16 & 6 & 0 & 2.553587 & 2.544065 & 0.782401 \\
\hline 17 & 6 & 0 & 1.674482 & 1.466395 & 0.719396 \\
\hline 18 & 1 & 0 & -0.984305 & 2.736356 & -0.604059 \\
\hline 19 & 1 & 0 & 0.544784 & 4.688628 & -0.882314 \\
\hline 20 & 1 & 0 & 2.867599 & 4.530774 & 0.015091 \\
\hline 21 & 1 & 0 & 3.465421 & 2.487747 & 1.339348 \\
\hline 22 & 6 & 0 & 0.745174 & 0.256755 & -1.224469 \\
\hline 23 & 1 & 0 & 0.325516 & 0.443613 & -2.190923 \\
\hline 24 & 6 & 0 & 1.926279 & -0.454623 & -1.475178 \\
\hline 25 & 6 & 0 & 3.164404 & 0.093283 & -1.113672 \\
\hline 26 & 6 & 0 & 1.869216 & -1.713926 & -2.087349 \\
\hline 27 & 6 & 0 & 4.306487 & -0.594717 & -1.356028 \\
\hline 28 & 1 & 0 & 3.208003 & 1.054794 & -0.646283 \\
\hline 29 & 6 & 0 & 3.011275 & -2.401939 & -2.329775 \\
\hline 30 & 1 & 0 & 0.923907 & -2.132226 & -2.363400 \\
\hline 31 & 6 & 0 & 4.249373 & -1.854069 & -1.968187 \\
\hline 32 & 1 & 0 & 5.251869 & -0.176448 & -1.079979 \\
\hline 33 & 1 & 0 & 2.967672 & -3.363460 & -2.797260 \\
\hline 34 & 1 & 0 & 5.151086 & -2.397245 & -2.159518 \\
\hline 35 & 6 & 0 & -1.657385 & -0.183949 & -0.057229 \\
\hline
\end{tabular}




$\begin{array}{llllll}36 & 6 & 0 & -2.500576 & -0.107719 & 1.003400 \\ 37 & 6 & 0 & -3.787038 & -0.185986 & 0.518427 \\ 38 & 1 & 0 & -2.217696 & 0.007380 & 2.028905 \\ 39 & 6 & 0 & -3.725792 & -0.341991 & -0.840785 \\ 40 & 6 & 0 & -4.990650 & -0.109673 & 1.209065 \\ 41 & 6 & 0 & -4.840418 & -0.336650 & -1.603673 \\ 42 & 6 & 0 & -6.171946 & -0.135635 & 0.447823 \\ 43 & 1 & 0 & -5.016788 & -0.029522 & 2.275699 \\ 44 & 6 & 0 & -6.095093 & -0.233076 & -0.963383 \\ 45 & 1 & 0 & -4.774787 & -0.414115 & -2.668810 \\ 46 & 1 & 0 & -7.124676 & -0.079428 & 0.931665 \\ 47 & 1 & 0 & -6.991989 & -0.230362 & -1.546904 \\ 48 & 8 & 0 & -2.363755 & -0.504085 & -1.287846\end{array}$

\section{$\mathrm{CF}_{3} \mathrm{COO}$}

Sum of electronic and zero-point Energies=

$-526.159642$

Sum of electronic and thermal Energies=

$-526.153721$

Sum of electronic and thermal Enthalpies=

$-526.152776$

Sum of electronic and thermal Free Energies=

$-526.190590$

Standard orientation:

\begin{tabular}{|c|c|c|c|c|}
\hline \multirow{2}{*}{$\begin{array}{l}\text { Center } \\
\text { Number }\end{array}$} & \multirow{2}{*}{$\begin{array}{l}\text { Atomic } \\
\text { Number }\end{array}$} & \multirow{2}{*}{$\begin{array}{l}\text { Atomic } \\
\text { Type }\end{array}$} & \multicolumn{2}{|c|}{ Coordinates (Angstroms) } \\
\hline & & & $x$ & Z \\
\hline 1 & 8 & -1.502892 & 1.145710 & -0.000574 \\
\hline 2 & 6 & -0.983566 & -0.063276 & -0.014675 \\
\hline 3 & 8 & -1.573388 & -1.113778 & -0.000684 \\
\hline 4 & 6 & 0.556656 & -0.009163 & -0.000430 \\
\hline 5 & 9 & 1.050761 & -1.085486 & -0.648419 \\
\hline 6 & 9 & 0.995247 & -0.012062 & 1.276335 \\
\hline 7 & 9 & 0.973071 & 1.117457 & -0.616729 \\
\hline
\end{tabular}

